Fat Crystal Spheroids - Formation, Characterization, Structure Modification, and use as Encapsulation Matrices

by

\title{
Tu Tran
}

M.Sc., Ryerson University, Toronto, Canada, 2011

B.Sc., Ryerson University, Toronto, Canada, 2008

A dissertation presented to Ryerson University

\author{
in partial fulfillment of the \\ requirements for the degree of \\ Doctor of Philosophy \\ in the program of \\ Molecular Science
}

Toronto, Ontario

CCopyright by Tu Tran 2016 


\section{Author's Declaration}

I hereby declare that I am the sole author of this dissertation. This is a true copy of the dissertation, including any required final revisions, as accepted by my examiners.

I authorize Ryerson University to lend this dissertation to other institutions or individuals for the purpose of scholarly research.

I further authorize Ryerson University to reproduce this dissertation by photocopying or by other means, in total or in part, at the request of other institutions or individuals for the purpose of scholarly research.

I understand that my dissertation may be made electronically available to the public. 
Fat Crystal Spheroids - Formation, Characterization, Structure Modification, and use as Encapsulation Matrices

\author{
Tu Tran \\ Ryerson University \\ Doctor of Philosophy \\ in the program of \\ Molecular Science \\ (C)2016
}

\begin{abstract}
Model fat systems consisting of fully-hydrogenated canola oil (FHCO) and canola oil (CO) were shear-crystallized using a rheometer with a parallel plate geometry at various cooling rates $\quad(0.2$ to $\left.5.0^{\circ} \mathrm{C} / \mathrm{min}\right)$ and shear rates $\left(0,500,1000\right.$, and $\left.2000 \mathrm{~s}^{-1}\right)$ to produce spheroidal fat crystals. These spheroids were characterized via rheology, polarized light microscopy (PLM), differential scanning calorimetry (DSC), and x-ray diffraction (XRD). Crystal spheroid formation was optimal at $1.0^{\circ} \mathrm{C} / \mathrm{min}$ and viscosity profiles followed a three phase sigmoidal shape. PLM analysis revealed that spheroid size decreased with increased shear rate while sphericity increased. A multi-step mechanism was proposed for the formation of these crystal spheroids.
\end{abstract}

Subsequently, different emulsifiers were used to modify the structure of these crystal spheroids and it was found that the type and concentration of emulsifier had significant effects on spheroid microstructure. Below a critical concentration, emulsifier could be incorporated into the crystal matrix of FHCO while above they would crystallize independently. DSC analysis revealed additional melting fractions compared to the control that were attributed to emulsifier 
incorporation and co-crystallization with FHCO. XRD showed that the crystallized spheroids were mainly of the $\beta^{\prime}$ polymorph regardless of the presence or type of emulsifier.

A water phase was then introduced within these systems to study their encapsulation potential. Emulsifier type significantly affected crystal shell morphology and encapsulation efficacy. The liquid-state emulsifiers (GMO and PGPR) showed limited interaction with FHCO, with GMO delaying the interfacial crystallization of FHCO while PGPR excluded FHCO from the droplet interface completely. Of the solid-state emulsifiers (GMS, GMP, SMS, and STS), the MAGs produced smooth-surfaced crystal shells around the emulsion droplets while the sorbitan-based emulsifiers produced irregularly-shaped shells and droplet cores (SMS) or incomplete crystal shell formation (STS).

The shear-crystallization of our model fat blend also resulted in the formation of cylindrical crystalline assemblies. The average diameter size of these crystal cylinders decreased with increased shear rate. A "log-rolling" mechanism was proposed for their formation.

These results demonstrate that laminar shear may be used to modify fat crystal microstructure and induce the formation of spheroidal and cylindrical crystalline assemblies. 


\section{Acknowledgements}

I would first like to thank my supervisor, Dr. Dérick Rousseau, for his guidance, insight, patience, and generosity throughout my doctoral studies. Always available and helpful, he provided many insights and great ideas, without which this work would not be possible.

I would also like to thank my thesis co-supervisor, Dr. Supratim Ghosh, and committee members, Drs. Darrick Heyd and Daniel Foucher, for providing valuable feedback and insight into my work.

For their help in various characterization techniques, I would like to thank Dr. Hassan Firoozmand (rheology) and Dr. Nicole Green (x-ray diffraction and microscopy).

I would like to thank all of my lab colleagues, especially Muhammad, Nicole, Ruby, Ryan, Yesudas, and the many international students for their helpful discussions and friendship during my research. They have made my doctoral experience a most memorable and enjoyable one.

I would like to say a special thank you to my family, especially my mom and dad, for always being there to support me and encourage me.

Finally, I would like to thank my wife, Yasmin, for her patience, love, and support. You are the one that kept me going. Thank you for believing in me.

Tu Tran 


\section{Table of Contents}

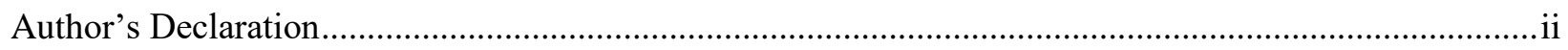

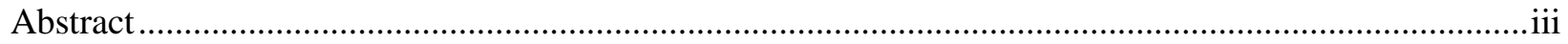

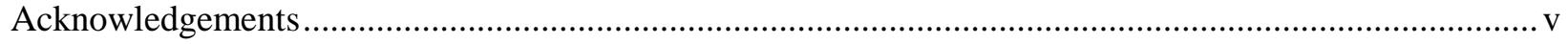

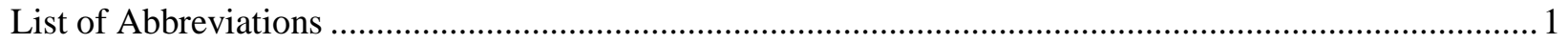

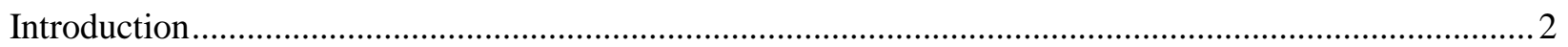

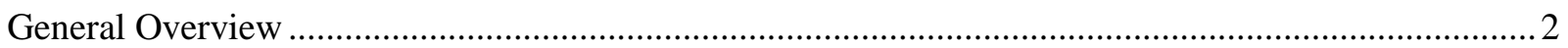

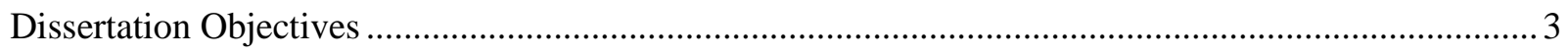

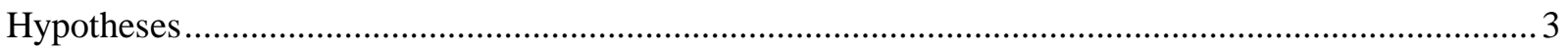

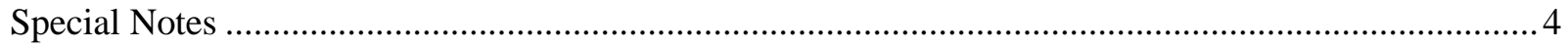

Chapter 1 - Influence of Shear on Fat Crystallization ...........................................................................5

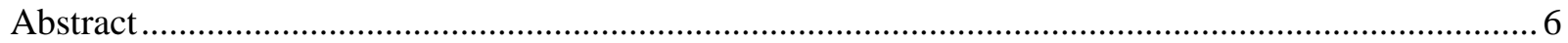

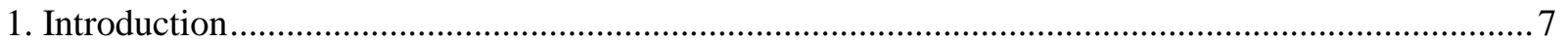

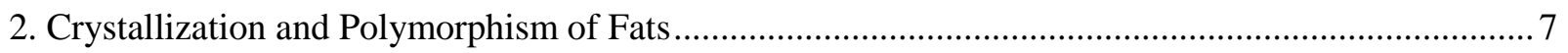

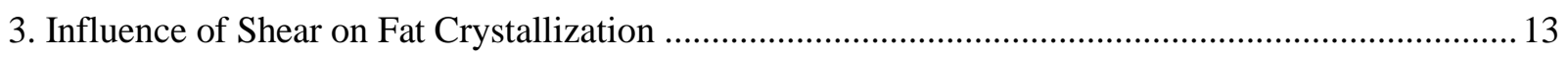

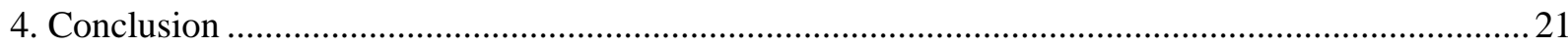

Chapter 2 - Spheroidal Fat Crystal Microstructures Formed with Confined Gap Shearing ......................23

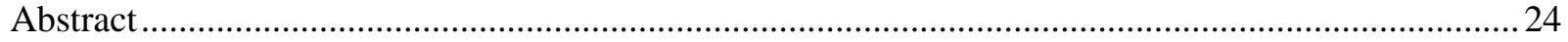

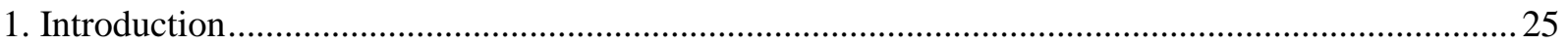

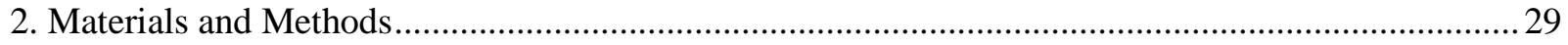

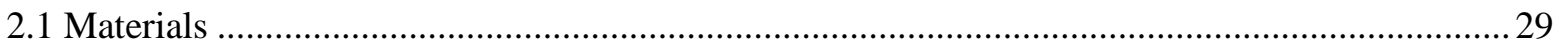

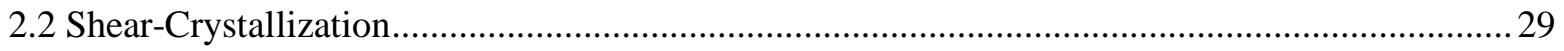

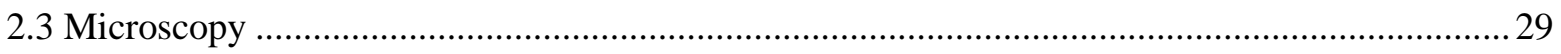

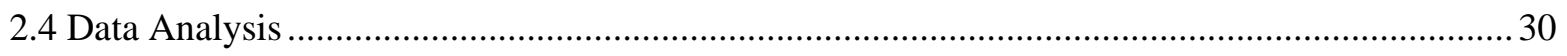

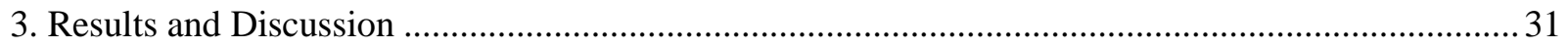

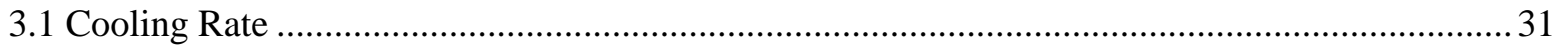

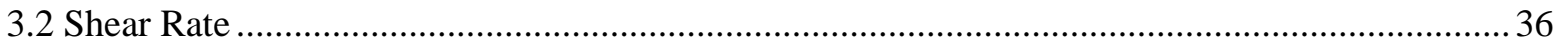

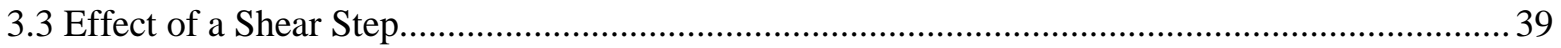

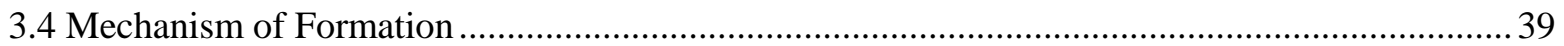

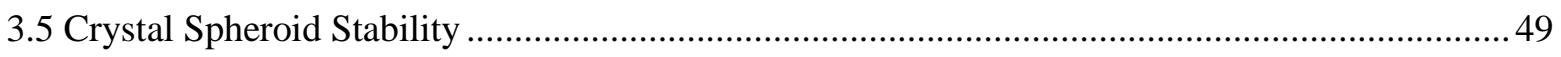

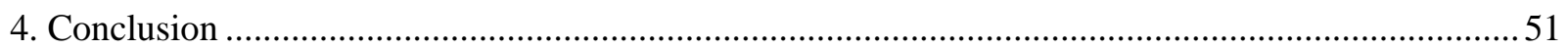


Chapter 3 - Spheroidal Fat Crystals: Structure Modification via use of Emulsifiers

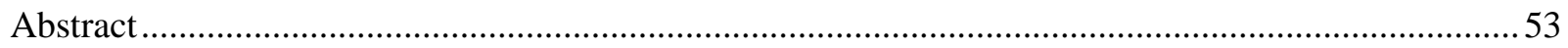

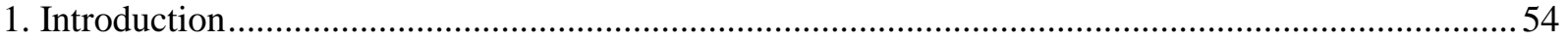

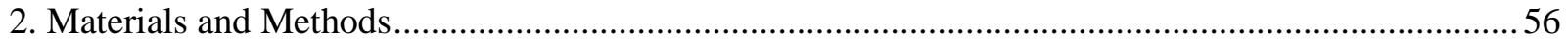

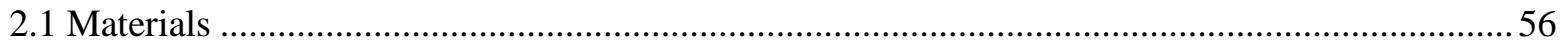

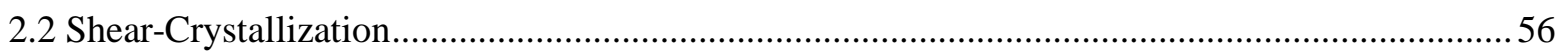

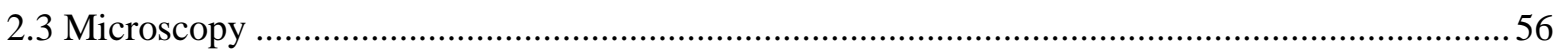

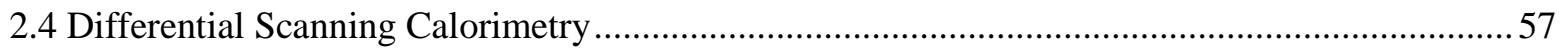

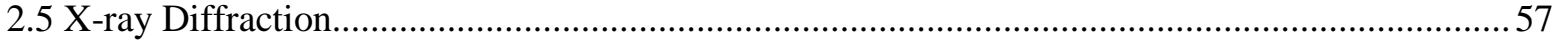

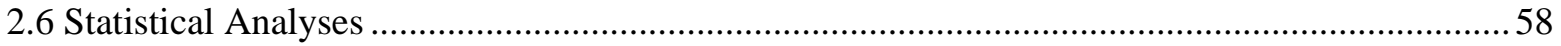

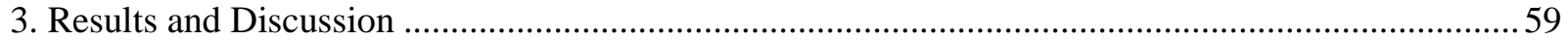

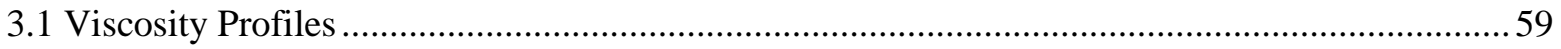

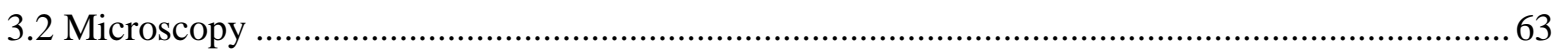

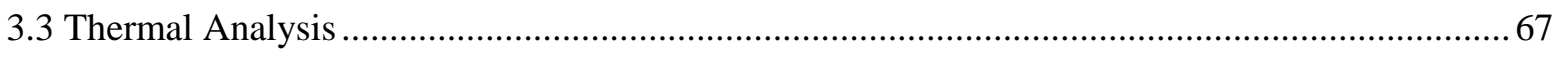

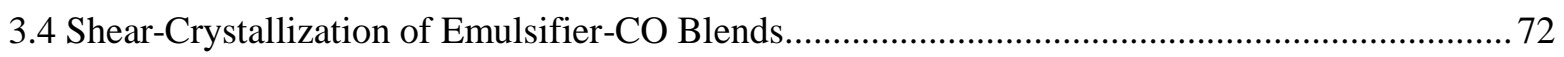

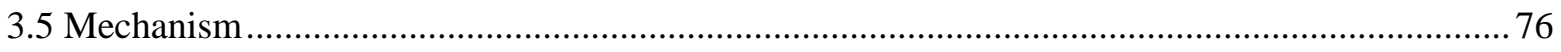

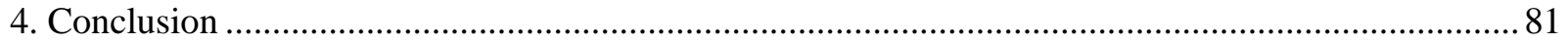

Chapter 4 - Encapsulation of Water-in-Oil Emulsion Droplets within Crystal Spheroids ........................82

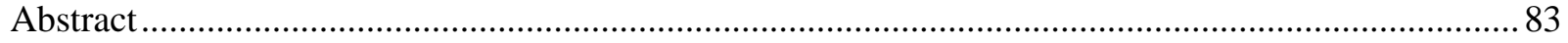

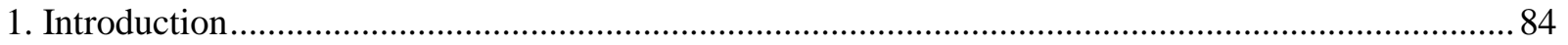

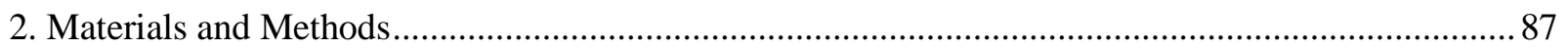

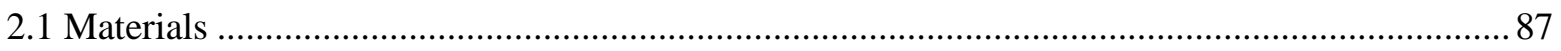

2.2 Emulsion Preparation and Shear-Crystallization ................................................................ 87

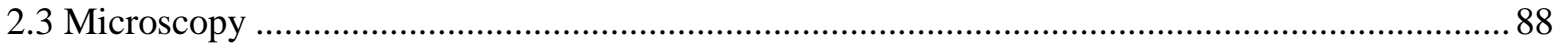

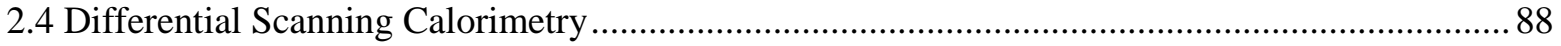

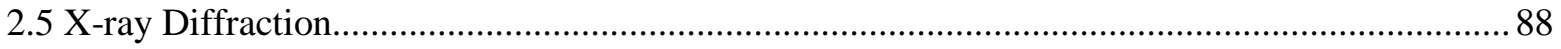

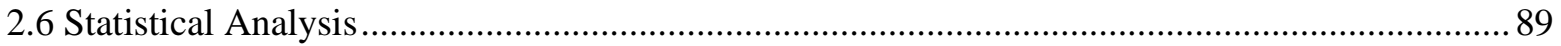

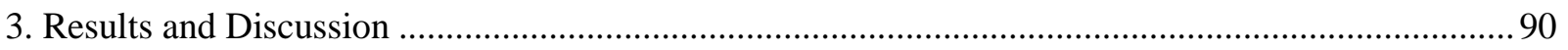

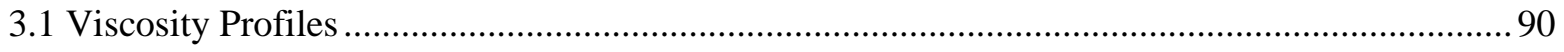

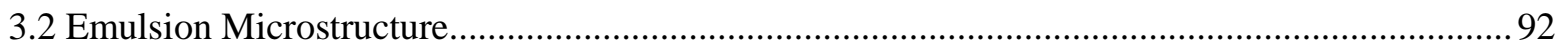

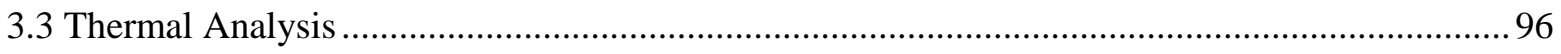

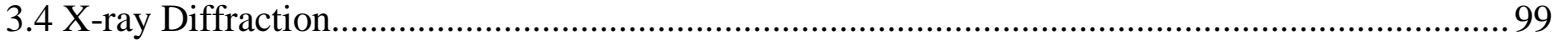




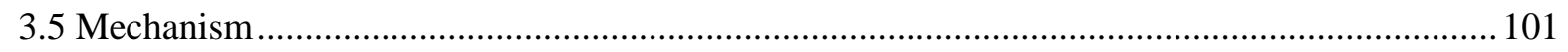

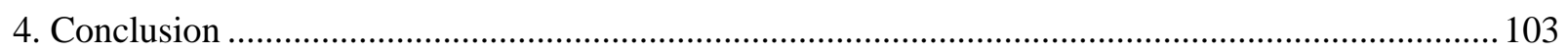

Chapter 5 - The Use of Shear to Produce Novel Crystal Microstructures ............................................. 104

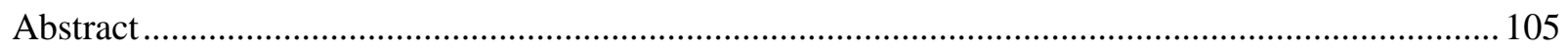

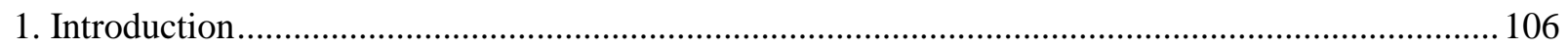

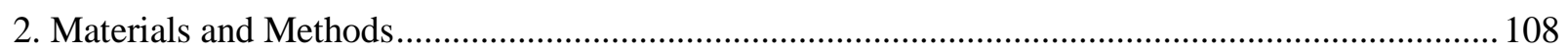

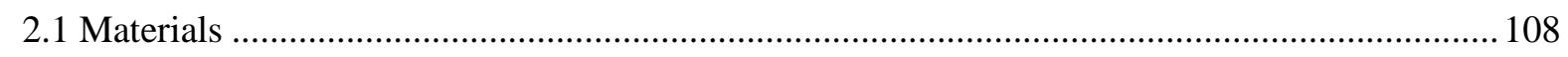

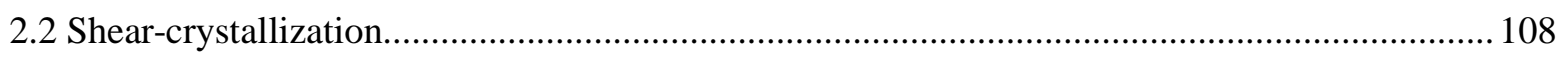

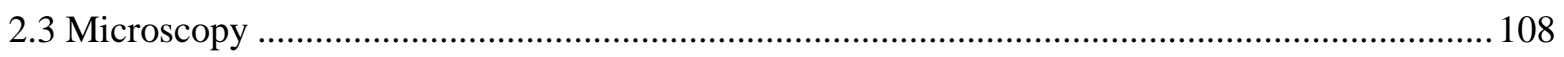

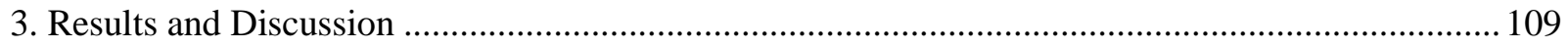

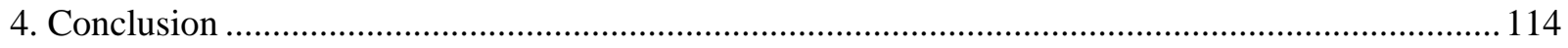

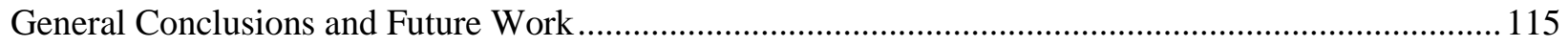

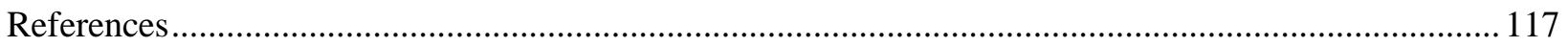




\section{List of Abbreviations}

$\begin{array}{ll}\text { CNP } & \text { Crystal nanoplatelet } \\ \text { CO } & \text { Canola oil } \\ \text { DAG } & \text { Diacylglycerol } \\ \text { DSC } & \text { Differential scanning calorimetry } \\ \text { FA } & \text { Fatty acid } \\ \text { FHCO } & \text { Fully-hydrogenated canola oil } \\ \text { GMO } & \text { Glycerol monooleate } \\ \text { GMP } & \text { Glycerol monopalmitate } \\ \text { GMS } & \text { Glycerol monostearate } \\ \text { MAG } & \text { Monoacylglycerol } \\ \text { PGFE } & \text { Polyglycerine fatty acid ester } \\ \text { PGPR } & \text { Polyglycerol polyricinoleate } \\ \text { PLM } & \text { Polarized light microscopy } \\ \text { PS } & \text { Palm stearin } \\ \text { SAXD } & \text { Small angle x-ray diffraction } \\ \text { SFC } & \text { Solid fat content } \\ \text { SMS } & \text { Sorbitan monostearate } \\ \text { SSHE } & \text { Scraped surface heat exchanger } \\ \text { STS } & \text { Sorbitan tristearate } \\ \text { TAG } & \text { Wiacylglycerol } \\ \text { WAXD } & \text { XRD }\end{array}$




\section{Introduction}

\section{General Overview}

There is a great amount of research dedicated to the study of fat crystallization, from nucleation and the formation of crystal nanoplatelets at the nanoscale to crystal growth and network formation at the mesoscale. Processing conditions such as cooling rate, crystallization temperature, and agitation (shear) may affect crystallization at various length scales. The effects of shear on fat crystallization are still not fully-understood. This dissertation focuses on the elucidation of the mechanism for crystal spheroid formation and the effects of various processing conditions on the shear-crystallization of model fat systems.

This dissertation begins with a review of fat crystallization and outlines the current work and knowledge of the effects of shear (chapter 1). Chapter 2 outlines the characterization of shearcrystallized model fat systems consisting of $\mathrm{FHCO}$ and $\mathrm{CO}$ where the optimal cooling and shearing conditions for the formation of the crystal spheroids were established along with a proposed multistep mechanism for their formation. Subsequently, the effects of emulsifiers on the formation of the spheroids was studied (Chapter 3) and it was found that emulsifier type and concentration had significant effects on spheroid morphology. Chapter 4 examines the introduction of a water phase as emulsion droplets and the use of the crystal spheroids as encapsulation matrices. Finally, chapter 5 examines the formation of fat crystal cylinders/frustums, a novel crystal microstructure, where a "log-rolling" mechanism was proposed for their formation. 


\section{Dissertation Objectives}

The overall objective of this dissertation was to characterize how processing conditions affected the formation of spheroidal fat crystals formed via the shear-crystallization of a model fat system within a narrow gap. The specific objectives were:

I. To test how cooling and shear rates affected crystal spheroid formation.

II. To characterize the physical properties of the spheroids.

III. To test how emulsifiers affect crystal spheroid formation.

IV. To apply shear-crystallization to emulsifier-containing emulsion systems for the encapsulation of droplets.

V. To elucidate the mechanism(s) of formation of the fat crystal spheroids.

\section{Hypotheses}

The proposed hypotheses were:

I. There would be optimal cooling and shear rates for the formation of the crystal spheroids.

II. Higher shear rates would result in smaller crystal spheroids.

III. The crystal spheroids would have different polymorphic, thermal, and rheological behaviour compared to statically-crystallized samples.

IV. Emulsifiers would significantly affect crystallization behaviour and spheroid formation.

V. Emulsifier-fat blends could be used to produce crystal spheroid-encapsulated water-in-oil emulsion droplets. 


\section{Special Notes}

All of the experimental work, except for acquisition of x-ray diffraction data which was collected by Dr Nicole Green, a post-doctorate fellow in Dérick Rousseau's lab, was performed by me. I was the primary author for all of the publications included in this dissertation.

The work in Chapter 1 has been submitted for publication while chapter 2 has been published (Tran, Ghosh, \& Rousseau, 2014) and chapter 3 has been accepted for publication in the journal Crystal Growth \& Design. 
Chapter 1 - Influence of Shear on Fat Crystallization 


\begin{abstract}
Processing conditions greatly impact fat crystallization kinetics and growth mechanisms. Recently, there has been increased interest in elucidating the role of shear on fat crystallization. This review provides an overview of fat crystallization under static conditions followed by a summary of the current body of work pertaining to the effects of shear on fat crystallization. The role of shear on different aspects of fat crystallization is reviewed: its effects on nucleation and growth, crystal network formation, polymorphic transitions, and microstructure modification at different length scales. The effects of shear are usually tied to critical shear rates but shear generally enhances nucleation and growth, accelerates crystallization and polymorphic transition times, and can cause orientation and structuring of fat crystal networks.
\end{abstract}




\section{Introduction}

Fats are important for many industries such as pharmaceutical, cosmetics, and food, and may be used as the chassis for products or as carrier matrices for the delivery of target compounds (Sato, 2001). The mechanical properties (e.g., texture, firmness, and spreadability), physical stability (e.g., phase separation), eating properties (e.g., mouthfeel or meltability), and visual appearance of the products depend on their underlying three-dimensional network of fat crystals. Fat composition, e.g., triacylglycerol (TAG) distribution and the presence of minor components such as emulsifiers, mono- and diacylglycerols (MAG and DAG, respectively) and/or non-fat ingredients, along with processing conditions such as cooling rate, agitation (shear), and processing temperature, may affect the crystallization behaviour of TAG molecules. There has been increased interest in elucidating the effect of shear on fat crystallization because of its industrial relevance, where it may be used to prevent sedimentation, aggregation, and enhance heat and mass transfer. Shear may also lead to the orientation of fat crystals and lead to the formation of new structured products. This review highlights recent research on fat crystallization with a focus on the effects of shear on fat structure at different length scales.

\section{Crystallization and Polymorphism of Fats}

Crystallization of fat occurs via nucleation and crystal growth. When molten fat is cooled to a temperature below the melting point of its highest-melting TAG, known as undercooling (usually at least $5-10{ }^{\circ} \mathrm{C}$ ), it will become supersaturated in the highest-melting TAG species and drive the system towards nucleation (Marangoni \& Wesdorp, 2013). The TAGs undergo constant fluctuation and movement within the melt due to thermal energy and Brownian motion, leading to dynamic association and dissociation. Spatial and energy localization fluctuations may lead to interactions of the correct molecular conformations required for the formation of stable crystal 
nuclei (Fig. 1.1). It has been postulated that there may be pre-structuring of TAG molecules into lamellar structures in the liquid state preceding nucleation, resulting in increased probability for the formation of stable nuclei (Hartel, 2001).

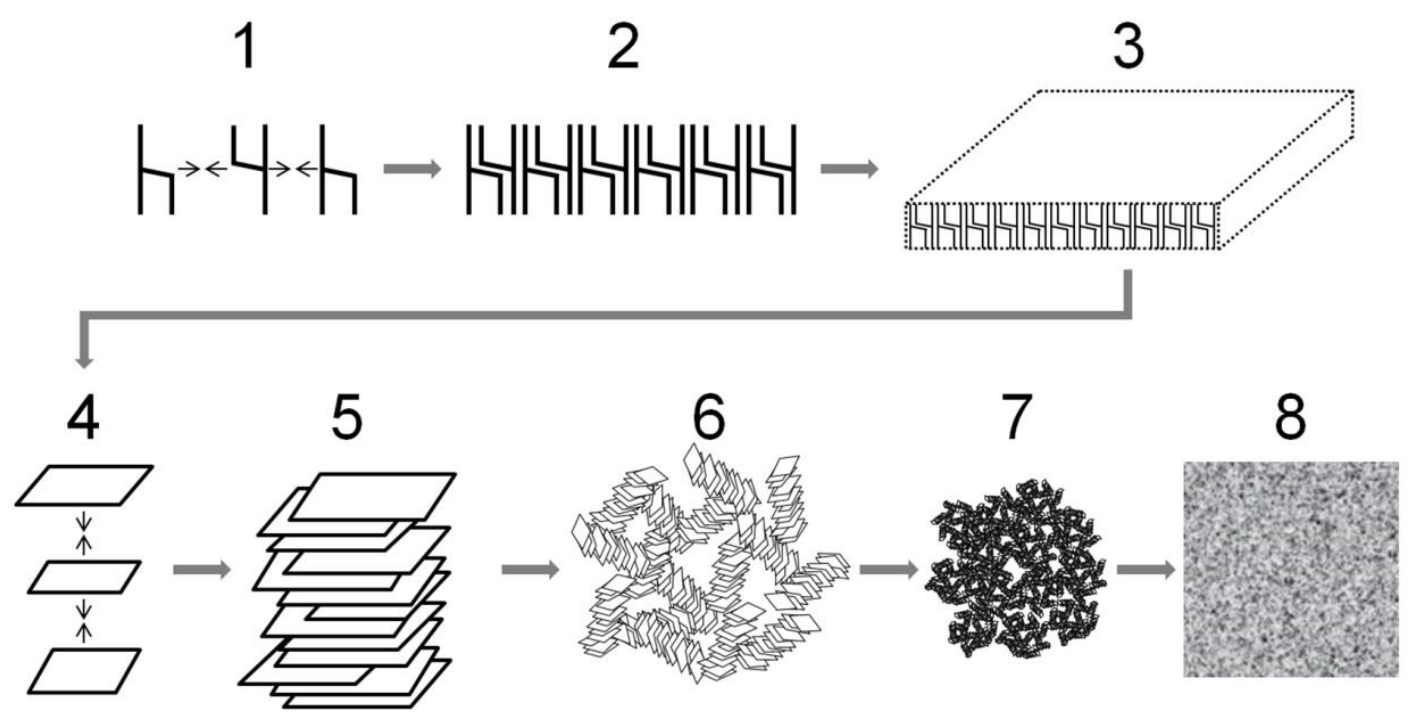

Figure 1. Structure hierarchy and multi-step crystallization of TAG: 1) Formation of stable nuclei; 2) Assembly into lamellae; 3) Association of lamellae to form crystal nanoplatelets (CNP); 4) Aggregation of CNP; 5) Formation of CNP stacks; 6) Random assembly of CNP stacks into crystallites; 7) Aggregation of crystallites into crystals; 8) Formation of 3D crystal network. Adapted from Marangoni and Wesdorp (Marangoni \& Wesdorp, 2013).

Nucleation can be divided into two categories: primary and secondary. Primary nucleation occurs via the spontaneous formation of nuclei from the melt and may be homogeneous (rare), as in a pure system consisting of only one type of TAG or within highly-dispersed nano-scale droplets, or heterogeneous (most common), where the presence of contaminants like dust or the coarse/pitted surface of the container vessel may act as foreign nucleating sites and induce nucleation. Secondary nucleation arises when minor components such as emulsifiers like MAG or DAG act as templating surfaces for TAG nucleation (Hartel, 2001; Marangoni \& Wesdorp, 2013; 
Walstra, 2003). Following the formation of stable nuclei, growth and association of crystallized lamellae result in the formation of crystal nanoplatelets (CNPs) (Acevedo \& Marangoni, 2010a, 2010b; Acevedo, Peyronel, \& Marangoni, 2011; Marangoni \& Wesdorp, 2013). These CNPs organize into stack-like structures by means of molecular van der Waals forces which then go on to assemble into crystallites that cluster via colloidal van der Waals forces to form crystals. Aggregation and sintering (formation of solid bridges) between crystals results in the formation of a 3D fat crystal network (Fig. 1) (Pink, Quinn, Peyronel, \& Marangoni, 2013; Tarabukina, Jego, Haudin, Navard, \& Peuvrel-Disdier, 2009).

The nature of the TAG [mono- or mixed fatty acids (FAs), chain length, degree of unsaturation] and processing conditions will affect how the TAG molecules pack and crystallize, resulting in different crystal polymorphs. The three main polymorphs that TAGs form, in order of increasing molecular organization, density, stability, and crystal size are $\alpha, \beta^{\prime}$, and $\beta$. In the $\alpha$-form, subcell packing arrangement is hexagonal $(\mathrm{H})$, where each TAG molecule is surrounded by six neighbours that may rotate along their long axis. The subcell packing of the $\beta^{\prime}$ form is orthorhombic $(\mathrm{O} \perp)$, where TAG chains lie perpendicularly to each other. With the $\beta$-form, the subcell is triclinic $\left(\mathrm{T}_{/ /}\right)$, with TAGs packed in parallel (Fig. 2A). The subcells are characterized by characteristic repeated distances between FA chains, known as short spacings, and can be measured via wide-angle x-ray diffraction or scattering (WAX). The nature of the TAG FAs will also impact their chain length structure, which is the repetitive sequence in the unit cell lamellae along the long-chain axis; it can exist in the double chain length (2L), i.e., two FA lengths, usually formed when the three FA of the TAG are the same or similar, or triple (3L) chain length formed when one FA is very different, i.e., when one FA is much shorter than the other two or contains an unsaturated cis-bond which causes a bend in the FA resulting in chain sorting (Fig. 2B). Chain length structures play an 
important role in the mixing behaviour between different TAG can be characterized via smallangle x-ray diffraction (SAX) (Sato, 2001; Walstra, 2003).

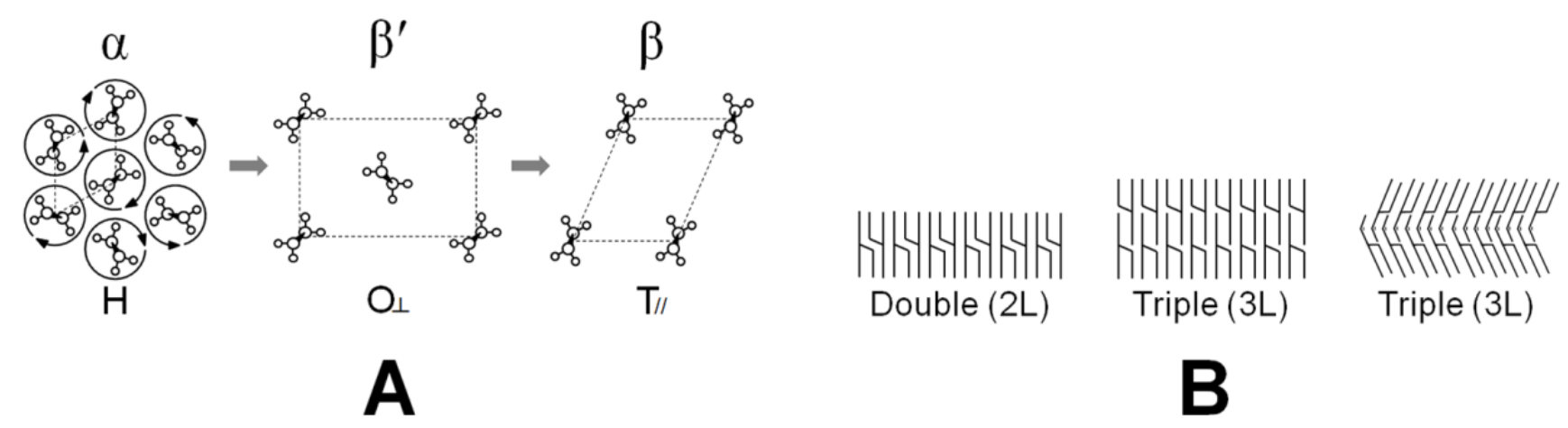

Figure 2. Common polymorphs (A) and chain length structures (B) of TAGs. Adapted from Marangoni and Wesdorp (Marangoni \& Wesdorp, 2013) and Sato (Sato, 2001).

Given that they are mixtures of varying TAGs, fats may exhibit a wide range of melting and crystallization temperatures and thus different polymorphs concurrently. The crystallization of a mixture of TAGs will usually lead to the formation of crystals that incorporate more than one type of TAG and are known as compound crystals. These compound crystals may form a solid solution but do not necessarily have to be homogeneous. That is, there may be groups of crystals with different TAG compositions. During crystallization, compound crystals form most easily as $\alpha$ crystals due to their high degree of freedom for molecular arrangement, minimal density restrictions and low relative activation energy of formation (Hartel, 2001; Marangoni \& Wesdorp, 2013; Sato, 2001). The lifespan of $\alpha$ crystals is typically relatively short and they will rapidly transition to the $\beta^{\prime}$, though this greatly depends on composition and processing conditions. Compound crystals may also form as $\beta^{\prime}$ crystals, but this does not occur as readily because of their higher packing densities and movement restrictions. $\beta$ crystals are never compound crystals 
(Walstra, 2003). Polymorphic transitions occur via the liquid state (solid-state transformations may occur, but typically on longer time scales) and happen most readily for mixtures of TAGs similar in structure (Walstra, 2003). In multicomponent fats with a wide compositional range, the lifetime of $\alpha$ crystals is much longer and $\beta^{\prime}$ crystals can exist almost indefinitely (Walstra, 2003).

Once formed, fat crystals may undergo aggregation leading to the formation of a continuous 3D network in an oil phase. The mechanical properties of these 3D networks depend on the chemical composition (types of TAGs and minor components such as emulsifiers) and processing conditions (Herrera \& Hartel, 2000). The formation of a fat crystal network usually occurs in steps: i) generation of a supersaturated state; ii) nucleation; iii) crystal growth; iv) recrystallization (polymorphic transformations); and v) formation of a 3D crystal network via the aggregation of crystals by means of diffusion-limited cluster aggregation due to van der Waals attractions, Brownian motion, and sintering (bridging between clusters by TAG crystals) (De Graef, Dewettinck, Verbeken, \& Foubert, 2006; Kloek, van Vliet, \& Walstra, 2005; Maleky, Smith, \& Marangoni, 2011). The latter three steps may occur concomitantly. Crystallization conditions such as cooling rate and thermal history (crystallization temperature and tempering processes) may have significant effects on the kinetics and physical properties of these crystallized systems (Pérez-Martínez, Alvarez-Salas, Charó-Alonso, Dibildox-Alvarado, \& Toro-Vazquez, 2007). Faster cooling rates favour the formation of $\alpha$ crystals while slower rates promote the formation of $\beta^{\prime}$ and $\beta$ polymorphs (Campos, Narine, \& Marangoni, 2002; Maleky, Acevedo, \& Marangoni, 2012). Faster cooling rates may also result in higher initial solid fat content (SFC) values and the formation of more numerous and smaller crystallites while slow cooling rates result in lower SFC and a smaller number of larger crystals (Acevedo \& Marangoni, 2010b). The degree of supercooling [i.e., decreasing the temperature below the crystallization temperature $\left(\mathrm{T}_{\mathfrak{c}}\right)$ of the 
TAG] may also influence the polymorphs of crystals formed. Isothermally crystallizing at lower temperatures increases the driving force for nucleation and promotes the formation of less-stable polymorphs such as the $\alpha$ form whereas isothermal crystallization at higher temperatures may result in solidification directly into more stable forms ( $\beta^{\prime}$ and $\beta$ ) (De Graef et al., 2006; Malssen, Peschar, \& Schenk, 1996; D. Rousseau, Hodge, Nickerson, \& Paulson, 2005).

The macroscopic properties of fat crystal networks such as mechanical strength and rheological properties (yield stress, viscosity, storage and loss moduli, etc.) depend on SFC and the morphology and interaction of crystals but not on crystal polymorph (Marangoni \& Rousseau, 1996; Narine \& Marangoni, 1999). Using fractal analysis of model fat systems, Marangoni et al. developed a model to relate the microscopic sub-unit building blocks of fat crystal networks, CNP, to their macroscopic mechanical properties (Marangoni et al., 2012; Tang \& Marangoni, 2006, 2007). They showed that fat crystals, with microstructures traditionally in the range of 1-200 $\mu \mathrm{m}$, are comprised of fractal aggregates of mesoscale CNP with typical dimensions in the hundred nanometer range (Acevedo \& Marangoni, 2010a, 2010b). The model approximates fat crystals as flocs of close-packed nearly-spherical clusters (spherulites) where the interaction between spherulites is stronger than the interaction between the relatively hard flocs ("weak-link" regime) and dictates the system's yield strength (Marangoni et al., 2012). 


\section{Influence of Shear on Fat Crystallization}

The development of fat crystal networks may be affected by shear (agitation), which can be applied in a number of ways such as mixing via an impeller or as laminar flow generated by Couette shear cells (Maleky et al., 2011), shear-processing cells (MacMillan \& Roberts, 2002), or the geometry attachments of a rheometer (Ghosh, Tran, \& Rousseau, 2011) (Fig. 3). Couette shear cells consist of two concentric cylinders between which a sample is deposited. The inner cylinder then rotates to generate shear. Shear processing cells operate in a similar fashion to parallel plate geometries used for rheometers: a sample is deposited between a stationary plate or stage and another plate that can rotate to generate shear. Understanding the effects of shear on nucleation and crystallization is relevant to industrial applications, where ingredients are usually sheared/mixed during processing to improve mass and heat transfer and enhance product homogeneity, and may improve process optimization and can lead to the development of novel products, e.g., tempering of chocolate to induce desired crystal polymorphs of cocoa butter to improve bloom resistance (Campos \& Marangoni, 2014; Stapley, Tewkesbury, \& Fryer, 1999) or the shear-processing of lipid shortenings to alter organoleptic properties such as hardness (Narine \& Humphrey, 2004).

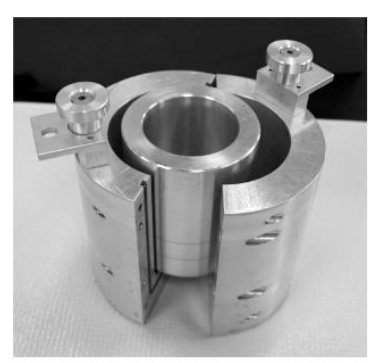

A

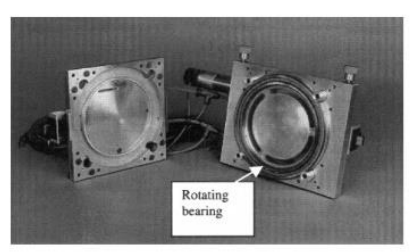

B

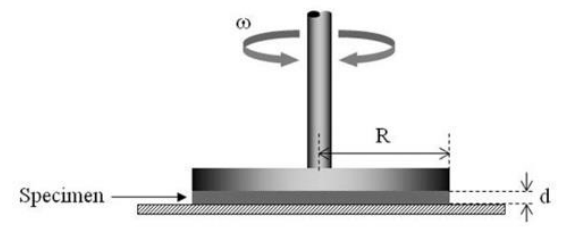

C

Figure 3. Instruments used for generating laminar shear: A) Couette shear cell [adapted from (Maleky et al., 2011)]; B) shear processing cell [adapted from (MacMillan \& Roberts, 2002)]; C) Parallel plate geometry of a rheometer 
Shear may increase the rate of primary nucleation by providing enough energy to overcome activation energy barriers for nucleation (Hartel, 2001). Increased nucleation results in a larger number of smaller crystals which typically translates into fat crystal networks with higher mechanical strength, though this depends on the aggregation behaviour of the component fat crystals (Marangoni \& Narine, 2002; Narine \& Marangoni, 1999). Additionally, shear may accelerate crystallization rates resulting in higher initial SFC values but over time, SFC will reach equilibrium values similar for statically-crystallized samples (Kaufmann, De Graef, Dewettinck, \& Wiking, 2012; Kaufmann, Kirkensgaard, Andersen, \& Wiking, 2013; Maleky \& Marangoni, 2008). Shear may also enhance secondary nucleation by fracturing newly-formed crystals and distributing them throughout the melt, providing increased seeds/interfaces for nucleation (Kloek et al., 2005; Maleky \& Marangoni, 2008). These effects are dependent on shear rate where, above a critical rate, the average size of crystals is reduced either through the breakage of larger crystals or inhibition of growth and aggregation of clusters (Kloek et al., 2005; Maleky et al., 2011). Below the critical shear rate, shear may increase the frequency of collision and contact time between crystallites, resulting in larger crystals (Tarabukina et al., 2009). Maleky et al., working on the shear-crystallization of cocoa butter, showed that shear can affect the nanostructure of CNPs with higher shear rates resulting in decreased CNP lengths and widths and a narrower size distribution compared to statically-crystallized samples (Maleky et al., 2012, 2011). Shear may affect CNP size at the nanoscale and crystal size on the meso-structural levels differently and a direct relationship between the different length scales is not readily apparent. That is, reduction in CNP size does not necessarily result in the reduction of meso crystals and vice versa (Acevedo \& Marangoni, 2014). 
Shear can greatly accelerate phase transitions to more stable polymorphs (Dhonsi \& Stapley, 2006; Sonwai \& Mackley, 2006), which MacMillan and Roberts hypothesized was due to the shear breaking weak van der Waals forces of lower polymorphs to allow restructuring into higher polymorphs (MacMillan \& Roberts, 2002). Mazzanti et al. postulated that shear forces provided enough energy to cause the melting of most of the crystal mass of lower polymorphs, leaving behind nuclei that could act as templates for growth into higher polymorphs (Mazzanti, Guthrie, Sirota, Marangoni, \& Idziak, 2003). In a different study, Mazzanti et al. suggested that shear may result in viscous heat generation that could produce enough heat to melt smaller, less-stable crystals but not larger crystals (Mazzanti, Li, Marangoni, \& Idziak, 2011). The accelerated polymorphic transitions may also be due to shear-structuring of the nanoplatelets formed upon nucleation which then act as templates for higher, more-organized polymorphs (Maleky et al., 2011). Kaufmann et al. studied the effect of shear and rapeseed oil addition on the polymorphic behaviour of milk fat via x-ray diffraction and showed that for isothermal crystallization, polymorphic transition times were accelerated by dilution with the oil with the explanation that the liquid oil increased gaps in the crystal network which allowed for conformational changes to occur more readily. Addition of oil also increased lamellar long-spacings and scaled with concentration. An interesting observation was that $\beta^{\prime}$ long spacings appeared before short spacings which indicated that the lamellar ordering preceded packing into the transverse crystalline form with increased dilution delaying this effect. The presence of shear accelerated polymorphic transitions to earlier times with effects being synergistic with oil dilution. Another notable finding during this study was that shear affected long spacings: statically-crystallized samples showed $2 \mathrm{~L}$ and $3 \mathrm{~L}$ packing but higher shear rates destroyed $3 \mathrm{~L}$ packing and left only $2 \mathrm{~L}$ packing. This shear effect was observed for oil-diluted blends but not pure AMF. Kaufmann et al. hypothesized that 
the addition of oil potentially changed the composition in the crystals which changed the average TAG chain length in the crystallizing fat (Kaufmann et al., 2013).

Mazzanti et al. found that shear can induce orientation of crystallites for a variety of fats (cocoa butter, milk fat, stripped milk fat and palm oil) (Mazzanti et al., 2003). Comparing the crystallization of native milk fat and stripped milk fat (no minor polar lipids), it was concluded that the minor polar lipids acted as impurities, which hindered crystallization events, stabilized the $\alpha$ form, and delayed polymorphic transformation to the more stable $\beta^{\prime}$ phase. Shear caused a reduction in crystal cluster sizes, but the extent was system-dependent. Low shear rates $\left(90 \mathrm{~s}^{-1}\right)$ resulted in little or no orientation while higher shear rates $\left(1440 \mathrm{~s}^{-1}\right)$ caused an appreciable degree of orientation in milk fat and cocoa butter but not palm oil. Instead of becoming oriented, the palm oil crystals formed spherical clusters that moved and tumbled in the shear field (Mazzanti et al., 2003). In a flowing system, the orientation of suspended particles depends on the interplay between shear, inter-particle, and Brownian forces. At low volume fractions, such as at the onset of nucleation and the early stages of crystallization, particles are separated by relatively large distances and inter-particle forces are negligible. The orientation of these particles may occur if the particles are above a critical size and if shear forces, which induce ordering, dominate over disorder-inducing Brownian forces (Kloek et al., 2005; Mazzanti et al., 2003). Mazzanti et al. also found that larger crystals are more readily-oriented than smaller ones and that higher shear rates resulted in increased thickness of nanoplatelets (Mazzanti et al., 2011). The introduction of a shear step, where the low initial shear rate of $90 \mathrm{~s}^{-1}$ was followed by an increase to $1440 \mathrm{~s}^{-1}$, increased nanoplatelet thickness further. It was postulated that the jump in shear rate caused melting of smaller platelets via a burst of viscous heating and allowed the larger platelets to grow via ripening (Mazzanti et al., 2011). 
The mode of shear application, such as turbulent flow delivered by impeller mixers and scraped surface heat exchangers (SSHE) or laminar flow by Couette-type cells and parallel plate shear cells, may have an impact on crystal microstructure and therefore fat plasticity. Acevedo and Marangoni tested the use of different methods of shear application using a SSHE, which provided turbulent flow and a Couette-type laminar shear cell on the crystallization of hydrogenated soy fat and soy oil blends (Acevedo \& Marangoni, 2014). Statically-crystallized samples showed expected spherulite microstructures while shear-crystallized samples, regardless of shear type, inhibited the formation of spherulites and promoted spherical clusters of needle-like crystals. Shearing caused an increase in nanoplatelet length with SSHE crystallization having greater impact than laminar shear-crystallization, which was contradictory to previously-reported results that shear decreased CNP size. This was attributed to the differences in shear rate between the studies and implied a critical shear rate below which CNP growth was enhanced whereas above this value crystal breakage and secondary nucleation were promoted (Acevedo \& Marangoni, 2014). Contrary to reports by Mazzanti that shear increases the thickness of nanoplatelets (Mazzanti et al., 2011), Acevedo and Marangoni did not find any correlation between shear and CNP thickness. Since Mazzanti et al. used the same Couette laminar shear cell, differences in the type of shear could be ruled out. Therefore, the differences in CNP thickness behaviour was probably due to the different shear rates used in the two studies: $\quad 1440 \mathrm{~s}^{-1}$ by Mazzanti et al. and $240 \mathrm{~s}^{-1}$ by Acevedo and Marangoni, which suggested that there is a critical shear rate for the generation of viscous heating. Mudge et al. examined the crystallization of cocoa butter under laminar shear using a Couette-type shear cell and found a critical shear rate for viscous heat generation (between 360 and $720 \mathrm{~s}^{-1}$ ) above which SFC decreased and polymorphic transitions were suppressed with effects being composition-dependent (i.e., samples with higher amounts of lower-melting TAGs were more 
susceptible to viscous heat-mediated melting) (Mudge \& Mazzanti, 2009). Also in their 2014 study, Acevedo and Marangoni reported decreased elastic modulus ( $\left.G^{\prime}\right)$ and yield stress $\left(\sigma^{*}\right)$ for sheared samples that had smaller crystal sizes, regardless of shear type, compared to staticallycrystallized samples, which was contradictory to reports that smaller crystals lead to higher mechanical strength (Campos et al., 2002; Narine \& Marangoni, 1999; Pérez-Martínez et al., 2007). They attributed the behaviour to differences in crystal growth mechanisms during storage, with the network growth of the sheared samples being inhibited by the confined flat 2D space of the microscope slide and cover slip vessel which was absent for previous studies (Acevedo \& Marangoni, 2014).

The combination of crystallization temperature, cooling rate, and shear can have significant impacts on crystal morphology and microstructure. Ghosh and Rousseau, while studying the effect of different surfactants on the interfacial crystallization of a model fat system [fully-hydrogenated canola oil (FHCO)] in canola oil (CO)], discovered that crystallizing the fat in the narrow gap $(<1$ $\mathrm{mm}$ ) between the parallel plates of a rheometer while applying laminar shear led to the formation of spheroidal crystal structures, which they dubbed "crystal cocoons" (Ghosh \& Rousseau, 2012). Acevedo et al. studied the effects of laminar shear on the structuring of fully-hydrogenated soybean oil as a function of shear rate and crystallization temperature and reported similar findings (Acevedo, Block, \& Marangoni, 2012). Shear-crystallization of this fat in the narrow gap (2.5 mm) of a Couette cell led to the formation of spheroidal crystal structures they referred to as "solid lipid meso-particles" (SLMs). It was found that SLM size was a function of shear rate: at an intermediate shear rate $\left(30 \mathrm{~s}^{-1}\right)$, larger SLMs formed while shearing at higher rates $\left(240 \mathrm{~s}^{-1}\right)$ resulted in smaller SLMs. This supported the existence of a critical shear rate, below which crystal aggregation and growth were promoted, possibly due to surface damage which led to their more rapid surface 
integration and enhanced crystal growth whereas shear rates above the critical value inhibited aggregation and even broke down crystals (Chaiseri \& Dimick, 1995; Garside \& Davey, 1980). There have been other incidental reports of shear-induced spheroidal TAG crystal assemblies. Herrera and Hartel used a paddle type mixer to study the crystallization behaviour of milk fat blends under agitation and revealed spheroidal crystal aggregates but only referred to them as crystals (Herrera \& Hartel, 2000). Lupi et al. studied the rheology of water-in-olive oil emulsions sheared via a rotor-stator setup and structured via MAGs and DAGs in combination with cocoa butter showed globular spherulitic aggregates with smoothed surfaces were generated at a shear rate of $100 \mathrm{~s}^{-1}$ (Lupi, Gabriele, De Cindio, Sánchez, \& Gallegos, 2011). De Graef et al. reported globular crystal structures of cocoa butter formed when sheared with a Couette-type shear cell at rates of 10 and $100 \mathrm{~s}^{-1}$ (De Graef, Van Puyvelde, Goderis, \& Dewettinck, 2009). Tarabukina et al. studied the crystallization and rheology of palm oil sheared via a rheometer with cone and plate as well as parallel plate geometries and demonstrated that shear rates of 10 and $100 \mathrm{~s}^{-1}$ could significantly impact microstructure and caused crystals to aggregate into nearly-spherical entities with higher density compared to statically-crystallized samples (Tarabukina et al., 2009).

Tran et al. worked to characterize and elucidate the mechanism of formation of these spheroidal crystals (Tran et al., 2014). Using a model fat system consisting of FHCO and CO and laminar shear generated using a rheometer with parallel plate geometry $\left(500,1000\right.$, and $\left.2000 \mathrm{~s}^{-1}\right)$, it was found that crystal spheroid formation was dependent on cooling rate, where an optimum/critical value was observed $\left(1.0^{\circ} \mathrm{C} / \mathrm{min}\right)$ above which spheroid formation was hindered due to the rapid crystallization of the fat resulting in less time for shear-induced organization and below which spheroid formation was also hindered due to crystal growth into higher polymorphs ( $\beta^{\prime}$ and/or $\beta$ ) being favoured, which presumably were not as susceptible to shear-induced aggregation compared 
to $\alpha$ crystals formed at slower cooling rates. Crystal spheroid size and viscosity decreased with higher shear rate, demonstrating that crystal size and morphology significantly impacted rheological properties, although these results may be counter-intuitive to the idea that more numerous smaller crystals would result in higher viscosity due to greater inter-particle interactions. A possible explanation would be that at the highest shear rate $\left(2000 \mathrm{~s}^{-1}\right)$ there was increased viscous heat generation that caused melting and polymorphic transitions at the solid spheroid-oil interfaces, leading to smoother surfaces and decreased surface area that decreased inter-particle contact and friction which resulted in lower viscosity. To elucidate the mechanism of crystal spheroid formation, shear crystallization was halted at various temperature and time points during development. Crystal microstructure was dependent on shearing time and crystallization temperature with spheroid development being a multistep process. The proposed mechanism for spheroid formation was: i) potential shear-induced pre-structuring of TAG in the melt; ii) nucleation and formation of nanoplatelets and subsequent CNP stacks; iii) orientation of CNP stacks parallel to the shear flow field below a critical shear rate or above which tumbling and rotation of nanoplatelet stacks occurred; iv) crystallization into bundles or clusters of $\alpha$ crystals; v) shear-accelerated polymorphic transitions to $\beta^{\prime} / \beta$; and vi) smoothing of spheroid surfaces due to viscous heat generation (Fig. 4) (Tran et al., 2014). 


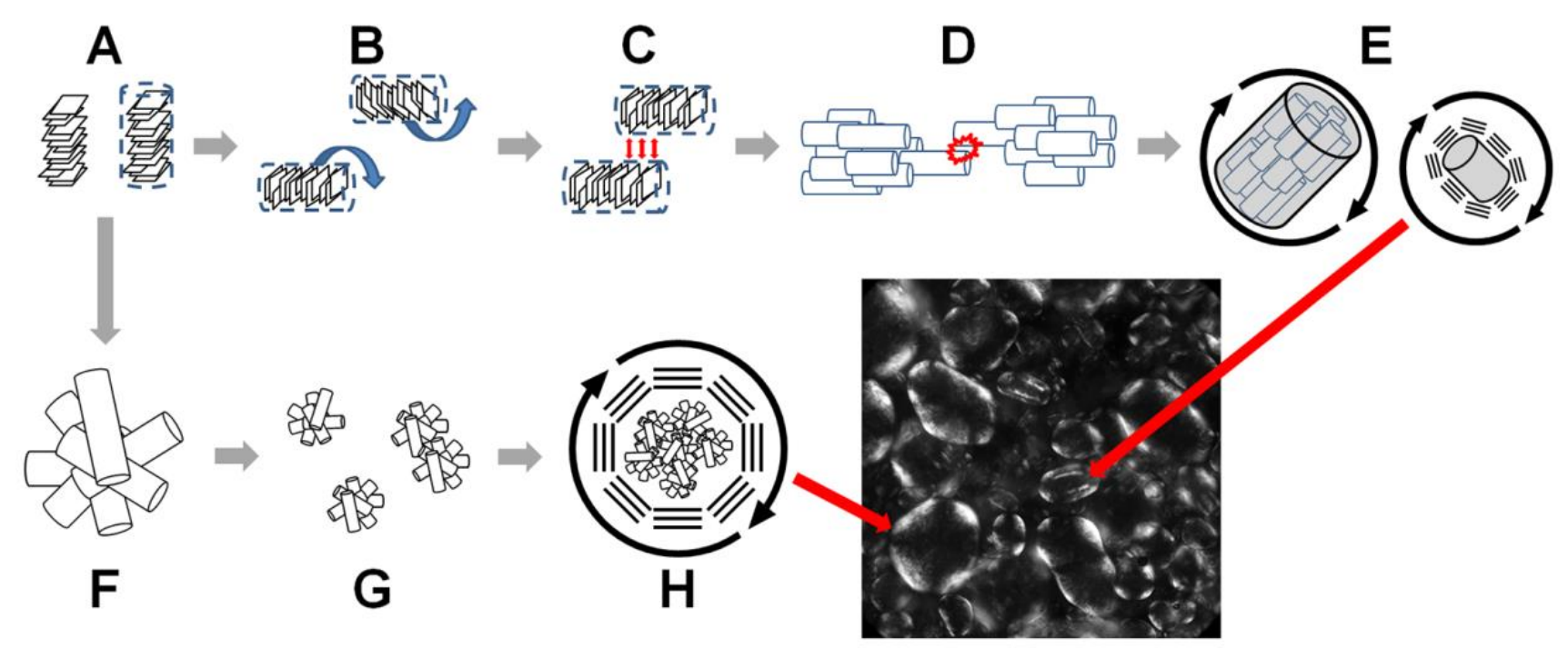

Figure 4. Proposed mechanism for crystal spheroid formation: A) CNP stack approximated by cylinders; B) Rotation of CNP cylinders and orientation parallel to shear field; C) Association of CNP cylinders; d) Formation of CNP cylinder bundles and breakage along weak points; E) Tumbling of CNP bundles and epitaxial growth; F) High shear-induced aggregation of CNP stacks into spherical clusters; G) Aggregation of spherical CNP clusters; H) Epitaxial crystal growth. Adapted from Tran et al. (Tran et al., 2014).

\section{Conclusion}

There have been a great number of studies on the crystallization of fats in an effort to understand the relationship between microstructural elements and macroscopic properties such as mechanical strength and organoleptic properties, all of which are dependent on the crystal networks which are susceptible to processing conditions such as cooling rate, temperature and agitation.

Recently, there have been increased efforts in elucidating the role of shear on fat crystallization with some general trends becoming apparent:

i. Shear greatly accelerates nucleation resulting in higher crystallization rates and an increased number of smaller crystals, which typically results in increased fat crystal network strength; 
ii. There exist system-dependent critical shear rates above which crystal growth is hindered due to the fracturing of larger crystals and inhibition of aggregation and clustering and below which crystallite aggregation is promoted, leading to larger crystals;

iii. Shear accelerates polymorphic transitions, possibly due to shear-induced viscous heat generation that melts lower polymorphs while leaving behind higher-ordered seeds;

iv. Shear affects crystallization at the nanoscale, causing decreased crystal nanoplatelet size at higher shear rates;

v. Crystals above a critical size may become oriented in the direction of the imposed shear field, and;

vi. Shear crystallization may be used to form new spheroidal crystal microstructures.

In closing, the effects of shear on fat crystallization are still not fully-understood and more work is required. Recent developments in instruments capable of concurrent shear and x-ray diffraction may be used in combination with electron microscopy techniques to elucidate crystal nanostructures and their shear-induced orientation with the goal of relating all of the different length scales. Understanding these properties will foster improvement of current fat-containing products and lead to the innovative new processed foods. 


\section{Chapter 2 - Spheroidal Fat Crystal Microstructures Formed with Confined Gap Shearing}
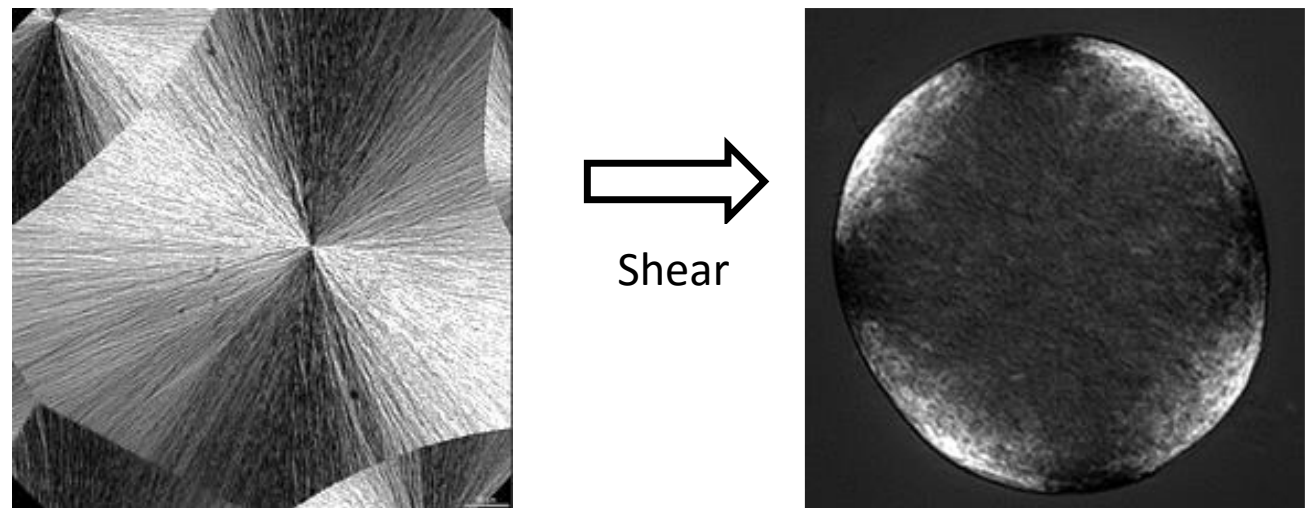

- Confined gap shear-cooling used to generate spheroidal fat crystal assemblies

- Simultaneous action of cooling and shear required for spheroid formation

- Mechanism of formation is a multi-scale, multiple step process

- Novel morphology may be used to control fat crystal network microstructure and rheology. 


\section{Abstract}

Confined gap shear-cooling was used to structure a model solid fat/liquid oil system consisting of fully-hydrogenated canola oil (FHCO) and canola oil (CO). Samples were cooled at various cooling rates $\left(0.2\right.$ to $\left.5.0^{\circ} \mathrm{C} / \mathrm{min}\right)$ and shear rates $\left(0,500,1000\right.$, and $\left.2000 \mathrm{~s}^{-1}\right)$ and characterized via polarized light microscopy and rheology. In the absence of shear $\left(0 \mathrm{~s}^{-1}\right)$, FHCO crystallized into an aggregated network of spherulites. Upon shearing, HCO crystallized into distinct spheroids whose size and structure were dependent on the cooling and shear rates used as well as crystallization duration and temperature. Lower shear rates resulted in larger, more irregularlyshaped spheroids whereas with higher shear rates these were more numerous and smaller. The simultaneous action of cooling and shear were required for spheroid formation, with shear having a greater impact. A mechanism was proposed to identify the multiple steps involved in the formation of the spheroids. Overall, these results demonstrated that it is possible to tailor the aggregation behaviour of individual fat crystals towards novel morphologies that may be used to control fat crystal network microstructure and rheology. 


\section{Introduction}

The mechanical properties (e.g., firmness and spreadability), eating properties (e.g., mouthfeel or meltability), physical stability (e.g., phase separation) and visual appearance of processed foods such as margarine and confectionery fillings are highly dependent on the underlying microstructure of their three-dimensional (3D) fat crystal network. Fat composition [i.e., triacylglycerol (TAG) make-up, presence of surfactants and non-fat ingredients, etc.] and processing conditions (cooling rate, crystallization temperature, time and shear) affect how TAG molecules crystallize and aggregate. In order of increasing stability, the three common crystal polymorphs in which TAGs exist are the $\alpha, \beta^{\prime}$, and $\beta$. The former has the lowest packing density and melting point whereas the latter have higher packing densities, stability, and melting points (Sato, 2001; Walstra, 2003).

The formation of a fat crystal network is a multi-step process. At temperatures below their crystallization temperature $\left(T_{c}\right)$, TAGs nucleate to form nanoplatelets that grow in size (Mazzanti et al., 2003). Under quiescent conditions, these nascent crystals aggregate via van der Waals attractions, Brownian motion, and sintering (formation of solid bridges between crystals) to form a 3D network via diffusion-limited cluster-cluster aggregation (De Graef et al., 2006; Kloek et al., 2005; Maleky et al., 2011).

Thermal history plays a significant role on the microstructure of crystallized fats (Pérez-Martínez et al., 2007). Faster cooling rates and greater supercooling often favour the formation of lessordered crystals (typically $\alpha$ crystals) whereas at slower rates, the formation of $\beta^{\prime}$ and $\beta$ crystals is typically promoted, given the lower driving force for crystallization (R. Campos et al., 2002; Maleky et al., 2012). Faster cooling rates may also result in a higher initial solid fat content (SFC) 
and the formation of more numerous, smaller crystallites with slower cooling rates yielding the opposite (Acevedo \& Marangoni, 2010b; Campos et al., 2002).

Mixing forces can greatly affect fat crystallization and network formation. Mixing as applied with an impeller-type arrangement may alter the aggregation behaviour as well as the structural and rheological properties of the resulting fat crystal network. The laminar flow generated within a narrow, confined gap [e.g., Couette shear cells (Maleky et al., 2011), shear-processing cells (MacMillan \& Roberts, 2002), or within the parallel plate geometry of a rheometer (Ghosh \& Rousseau, 2012)] may result in alteration of crystallization at smaller length scales, resulting in accelerated polymorphic phase transitions to more stable polymorphs (MacMillan \& Roberts, 2002). Mazzanti et al. found that sufficiently high shear forces provided enough viscous heat to melt lower-melting crystals, leaving behind nuclei that could act as templates for growth into higher-order polymorphs (Mazzanti et al., 2003). Such heating also reduced the overall size of crystal clusters, either through the fracturing of larger crystals or by enhancing nucleation while inhibiting cluster growth and aggregation (Acevedo et al., 2012; Kloek et al., 2005; Maleky et al., 2011). Mazzanti et al. also found that shear induced orientation of crystallites in a variety of fats (cocoa butter, milk fat, and palm oil), with shear at $90 \mathrm{~s}^{-1}$ resulting in little or no orientation whereas shear at $1400 \mathrm{~s}^{-1}$ caused an appreciable degree of orientation (Mazzanti et al., 2003).

Phenomenologically, the orientation of suspended particles in a flowing system depends on numerous factors, including the presence of shear, inter-particle interactions and Brownian motion. At low volume fractions, such as during nucleation onset, inter-particle forces are negligible given the low number-concentration of nuclei. Therefore, particle orientation depends on the interplay of order-inducing (shear) and disorder-inducing (Brownian) forces. Shear causes particles to rotate slowly when they are oriented nearly parallel to the direction of flow and faster when perpendicular 
to the flow, resulting in preferential orientation parallel to the flow field with time. In the context of fat crystallization, larger crystals would be more readily oriented than smaller ones, given they are more affected by the flow field and experience greater shear effects (Mazzanti et al., 2011). When combined, the processing conditions discussed above clearly impact crystal morphology and microstructure.

There have been incidental reports of sphere-like TAG crystalline assemblies formed as a result of cooling under stirring. Herrera and Hartel (2000) studied the bulk crystallization of milkfat blends under agitation, and revealed the presence of fat crystal spheroidal aggregates, though they only referred to them as crystals (Herrera \& Hartel, 2000). Lupi et al. (2011) studied the rheology of water-in-olive oil emulsions structured via crystallization of partial acylglycerols and cocoa butter and showed that smoothed spherulitic aggregates were generated at a shear rate of $100 \mathrm{~s}^{-1}$ (Lupi et al., 2011). De Graef et al. (2008) found that shear enhanced the primary nucleation and crystallization of palm oil, with shear rates of $10^{-1}$ and $100 \mathrm{~s}^{-1}$ leading to the formation of globular 'crystal structures' (De Graef et al., 2009). Tarabukina et al. (2009) investigated the impact of shear on palm oil crystallization during cooling (Tarabukina et al., 2009). Shear significantly influenced spherulite size and growth rate of the palm oil with steady-state structures consisting of aggregated spherulites, demonstrating the importance of thermo-mechanical history on fat crystal aggregation. More recently, Acevedo et al. found that shear-cooling fully hydrogenated soybean oil in the narrow gap $(2.5 \mathrm{~mm})$ of a Couette cell led to the formation of spheroid crystal structures whose size was a function of shear rate $\left(30 v s .240 \mathrm{~s}^{-1}\right)$ (Acevedo et al., 2012). Temperature had a more pronounced effect at $30 \mathrm{~s}^{-1}$ than at $240 \mathrm{~s}^{-1}$ with larger structures formed at higher temperatures. They suggested that below a critical shear rate, crystal aggregation was promoted while shear rates above this critical value inhibited aggregation and potentially broke down crystal 
assemblies. Finally, Ghosh and Rousseau found that shear-crystallizing fat-stabilized water-in-oil emulsions in the narrow gap $(<1 \mathrm{~mm})$ between the parallel plates of a rheometer led to the formation of spheroidal crystal structures that encapsulated the dispersed aqueous droplets (Ghosh \& Rousseau, 2012).

The literature indicates that the factors and mechanisms required to form spheroidal structures are not yet fully understood. In this study, we explored the juxtaposition between cooling and shear rate on spheroid formation and elaborated on existing mechanisms possibly responsible for their formation. To do so, confined gap shear-cooling was used to structure mixtures of solid fat and liquid oil at various cooling and shear rates. We show that the simultaneous action of cooling and shear were required to form fat crystal spheroids, with shear having a greater impact. 


\section{Materials and Methods}

\subsection{Materials}

Canola oil (acid value $\sim 0.2 \%$ ) was purchased from a local grocery store and stored at room temperature. Fully-hydrogenated canola oil (FHCO) was purchased from Bunge (Oakville, ON, Canada). It had a capillary melting point of $69.5^{\circ} \mathrm{C}$ (AOCS official method Cc 1-25) and a free fatty acid content of $0.018 \%$ (AOCS Society, 1998). The predominant TAG species in FHCO were $\mathrm{C}_{52}(13.3 \%), \mathrm{C}_{54}(73.6 \%)$, and $\mathrm{C}_{56}(5.0 \%)$.

\subsection{Shear-Crystallization}

A $10 \mathrm{wt} \%$ FHCO solution in canola oil was heated to $70{ }^{\circ} \mathrm{C}$ with continuous stirring to ensure complete melting of the FHCO. Aliquots were deposited between the parallel plates (PP25, 25 mm diameter, $0.5 \mathrm{~mm}$ gap) of a rheometer (Physica MCR301, Anton Paar, Ville St-Laurent, QC,

Canada). Samples were then maintained at $70{ }^{\circ} \mathrm{C}$ for $20 \mathrm{~min}$ and then cooled at

${ }^{\circ} \mathrm{C} /$ min while being sheared at a constant rate ranging from $0-2000 \mathrm{~s}^{-1}$. The apparent viscosity of the samples was measured using the rheometer and measurements were recorded as samples were sheared and cooled. All experiments were performed at least in triplicate.

\subsection{Microscopy}

The microstructure of samples was observed via polarized light microscopy (PLM). Samples were placed on viewing slides at room temperature $\left(\sim 25^{\circ} \mathrm{C}\right)$ (Fisher Scientific, Ottawa, ON, Canada), covered with a cover slip (Fisher Scientific, Nepean, ON, Canada) and analyzed with an inverted light microscope (Axiovert 200M, Zeiss Inc., Toronto, ON, Canada) equipped with a CCD camera 
(Q Imaging, RETIGA 32-0012B-105, Surrey, BC, Canada) using Northern Eclipse imaging software (Version 7.0, Empix Imaging Inc., Mississauga, ON, Canada).

2.4 Data Analysis: All results reported are the arithmetic mean \pm standard deviation of triplicate experiments. Statistical analysis was performed using either a two-tailed student's $t$-test (for single pair comparisons) or one-way ANOVA with single-step Tukey's multiple comparison post-hoc test for multiple groups. Differences were considered statistically significant at $\mathrm{P} \leq 0.05$. 


\section{Results and Discussion}

\subsection{Cooling Rate}

Fig. 1 reports the apparent viscosity of the $\mathrm{FHCO} / \mathrm{CO}$ blends sheared at $2000 \mathrm{~s}^{-1}$ as a function of temperature $\left(70\right.$ to $20{ }^{\circ} \mathrm{C}$ ) at 5 cooling rates ranging from $0.2-5^{\circ} \mathrm{C} / \mathrm{min}$. A three-phase viscosity profile (I-III) was apparent at all cooling rates. From a molecular perspective, the increase in TAG melt shear viscosity (phase I) was attributed to the increased interaction between neighbouring TAGs as the temperature was reduced. Dibildox-Alvarado et al. found evidence of van der Waalsmediated interactions responsible for pre-structuring and microviscosity changes in liquid-state TAG blends (Dibildox-Alvarado, Laredo, Toro-Vazquez, \& Marangoni, 2010). Higher cooling rates resulted in faster increases in apparent viscosity. The temperatures at the end of phase I decreased with increasing cooling rate $(\mathrm{P} \leq 0.05)$, suggesting an effect on pre-nucleation liquidstate TAG-TAG interactions. At slower cooling rates (e.g., $0.2^{\circ} \mathrm{C} / \mathrm{min}$ ), with more time for TAGs to organize in the melt, there would be a lower driving force for nucleation and a higher drive for crystal growth likely resulting in the formation of higher-order polymorphs $\left(\beta / \beta^{\prime}\right)$ and eventually spherulites. At faster cooling rates, the TAGs presumably formed less-dense $\alpha$ crystals that formed clusters more susceptible to shear-induced tumbling, resulting in the formation of crystal spheroids. 


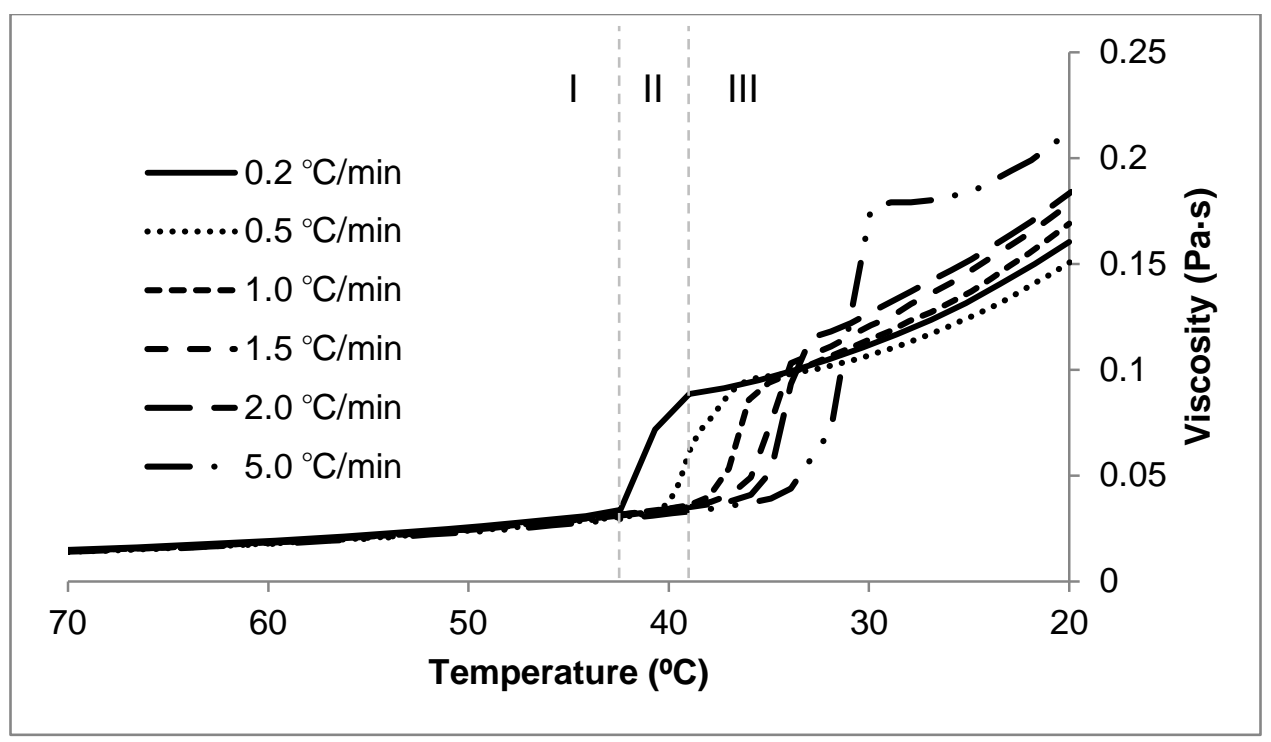

Figure 1. Viscosity profiles of $10 \mathrm{wt} \% \mathrm{FHCO}$ and $90 \mathrm{wt} \% \mathrm{CO}$ blends sheared at $2000 \mathrm{~s}^{-1}$ and crystallized at different cooling rates. Error bars removed for clarity $(n=3)$.

The second phase of crystallization (II), shown as the sharp increase in apparent viscosity within a narrow temperature range, resulted from the initial crystallization of FHCO. The rate of increase in viscosity for this second phase significantly differed with cooling rate (Table 1$)(\mathrm{P} \leq 0.05)$. There were also significant differences in crystallization temperature $\left(\mathrm{T}_{\mathrm{c}}\right)$ (the temperature at the end of phase I) between the different cooling rates (Table 1) $(\mathrm{P} \leq 0.05)$, which was also attributed to increased supercooling effects. The apparent viscosity at the end of phase II increased with faster cooling rates, presumably due to decreased induction times which resulted in a higher fraction of the FHCO crystallizing. 
Table 1. Rates of increase in viscosity and changes in crystallization temperature $\left(T_{c}\right)$ at different cooling rates (units of $\mathrm{Pa} \cdot \mathrm{s} /{ }^{\circ} \mathrm{C}$ ). Rates have been multiplied by a factor of 100 for formatting and are reported as means \pm standard deviations $(\mathrm{n}=3)$.

\begin{tabular}{ccccc}
$\begin{array}{c}\text { Cooling rate } \\
\left({ }^{\circ} \mathrm{C} / \mathrm{min}\right)\end{array}$ & Phase I & Phase II & Phase III & $\begin{array}{c}\mathrm{T}_{\mathrm{c}} \\
\left({ }^{\circ} \mathrm{C}\right)\end{array}$ \\
\hline 0.2 & $0.06 \pm 0.00$ & $2.02 \pm 0.60$ & $0.37 \pm 0.05$ & $42.4 \pm 0.0$ \\
0.5 & $0.06 \pm 0.01$ & $1.37 \pm 0.19$ & $0.49 \pm 0.03$ & $41.2 \pm 1.1$ \\
1.0 & $0.06 \pm 0.00$ & $1.45 \pm 0.05$ & $0.49 \pm 0.02$ & $38.9 \pm 0.0$ \\
1.5 & $0.06 \pm 0.00$ & $1.90 \pm 0.10$ & $0.45 \pm 0.14$ & $37.2 \pm 0.0$ \\
2.0 & $0.06 \pm 0.01$ & $2.47 \pm 0.26$ & $0.42 \pm 0.04$ & $35.4 \pm 0.0$ \\
5.0 & $0.06 \pm 0.01$ & $2.42 \pm 0.83$ & $0.38 \pm 0.03$ & $36.2 \pm 1.3$ \\
\hline
\end{tabular}

The phase III viscosity profile was attributed to the combination of continued crystal growth and the $\alpha \rightarrow \beta^{\prime}$ or $\beta$ polymorphic transition of the FHCO (Mazzanti, Marangoni, \& Idziak, 2005). De Graef et al. reported similar increases in apparent viscosity for the crystallization of palm oil under shear and attributed it to polymorphic transitions, crystal growth as well as direct melt-to- $\beta$ ' crystal formation (De Graef et al., 2009). The duration of phase III decreased with faster cooling rates given the higher proportions of FHCO crystallizing during phase II and the consequent reduction in FHCO remaining in the melt. The clear break between phases II and III implied that after first crystallizing into lower polymorphs, there was structural reorganization of TAG molecules into higher polymorphs.

Although all cooling rates showed similar three-phase crystallization patterns, the resulting microstructure drastically differed (Fig. 2). An optimal cooling rate of $1.0^{\circ} \mathrm{C} / \mathrm{min}$ resulted in the highest number of spheroids formed whereas at $1.5{ }^{\circ} \mathrm{C} / \mathrm{min}$, there was also evidence of nonspheroid crystals. The spheroids generated at these two cooling rates bore the smoothest surfaces. At higher cooling rates, no spheroidal assemblies were observed, i.e., at $5{ }^{\circ} \mathrm{C} / \mathrm{min}$, the $\mathrm{FHCO}$ crystallized and aggregated to form a $3 \mathrm{D}$ network whereas at $2{ }^{\circ} \mathrm{C} / \mathrm{min}$, crystal-crystal clustering 
was observed but did not lead to spheroid formation. Samples cooled at $0.5^{\circ} \mathrm{C} / \mathrm{min}$ also showed evidence of spheroidal shapes; however there was concurrent crystallization in the continuous phase, indicating simultaneous spheroid/non-spheroid formation. At $0.2^{\circ} \mathrm{C} / \mathrm{min}$, FHCO strictly crystallized as spherulites.

The possibility that these morphological differences were due to unequal processing conditions resulting from different cooling rate-dependent shearing times was rejected as experiments with matched shearing durations did not yield such disparities. This suggested that once the FHCO had crystallized as spherulites, it was not possible to transform them into spheroids by imposing further shear. 

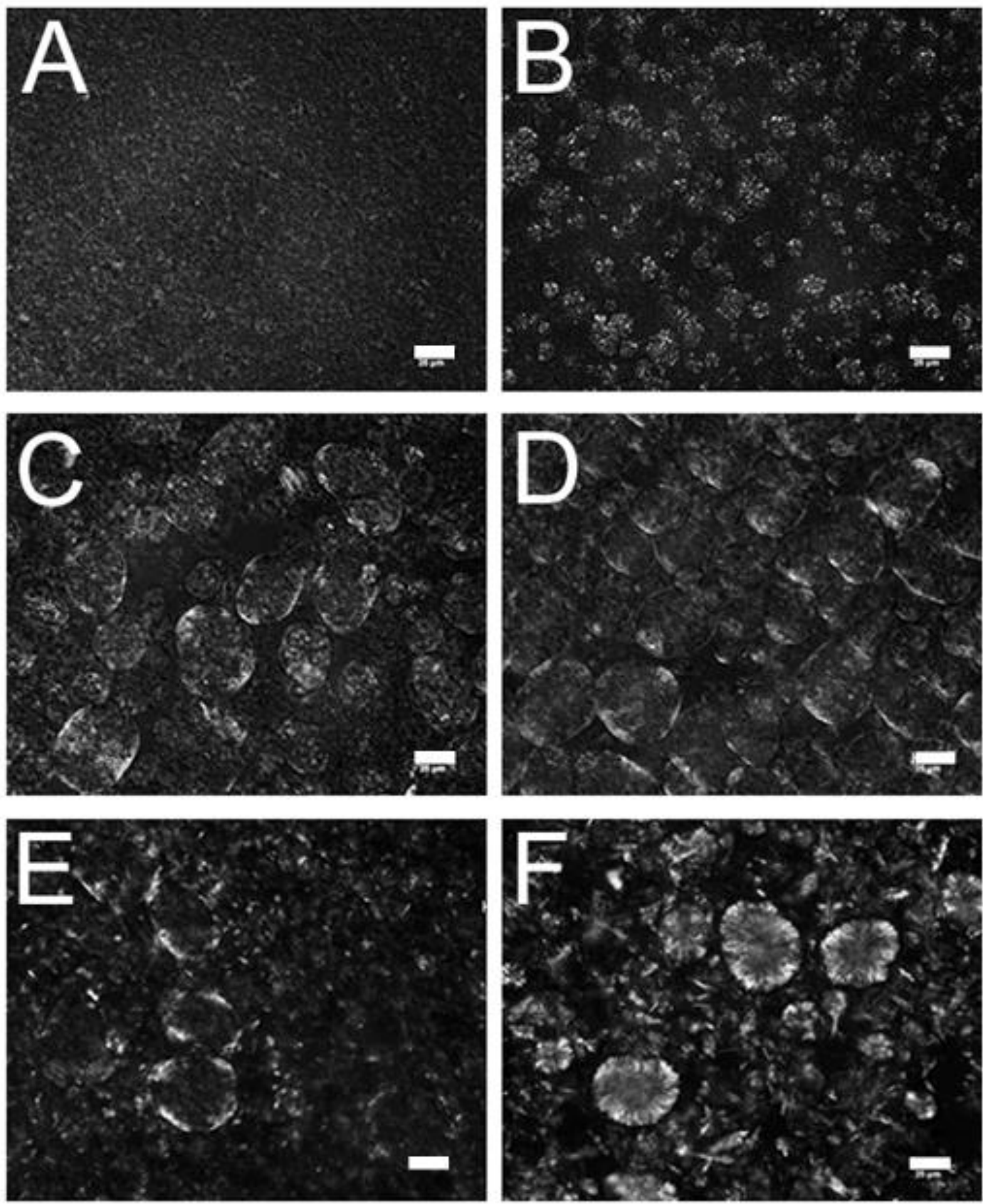

Figure 2. Microstructure of FHCO crystallized (shear rate $=2000 \mathrm{~s}^{-1}$ and gap size $=0.5 \mathrm{~mm}$ ) at different cooling rates: A) $\left.5.0{ }^{\circ} \mathrm{C} / \mathrm{min}, \mathrm{B}\right) 2.0{ }^{\circ} \mathrm{C} / \mathrm{min}$, C) $\left.\left.1.5{ }^{\circ} \mathrm{C} / \mathrm{min}, \mathrm{D}\right) 1.0{ }^{\circ} \mathrm{C} / \mathrm{min}, \mathrm{E}\right) 0.5^{\circ} \mathrm{C} / \mathrm{min}$, F) $0.2{ }^{\circ} \mathrm{C} / \mathrm{min}$. Size bar represents $25 \mu \mathrm{m}$. 


\subsection{Shear Rate}

Fig. 3 shows that for samples cooled at $1.0^{\circ} \mathrm{C} / \mathrm{min}$ using three shear rates $(500,1000$, and 2000

$\mathrm{s}^{-1}$ ), there were no significant effects on the viscosity profile rate constants for phases I and II $(\mathrm{P}>0.05)$. However, the different shear rates had a significant effect on the phase III rate constants and final apparent viscosities $(\mathrm{P} \leq 0.05)$. Hence, shear likely did not affect pre-nucleation intermolecular TAG interactions and $\alpha$-form crystallization, but did affect the polymorphic transitions and continued growth thought to occur during phase III. Similar shear rate-dependent results were found for samples cooled at 0.5 and $1.5^{\circ} \mathrm{C} / \mathrm{min}$. Notably, at lower shear rates $\left(0.5^{\circ} \mathrm{C} / \mathrm{min}\right.$ and 500 $\mathrm{s}^{-1}$ ), the spheroids were larger and more irregularly-shaped (Fig. 4A) whereas at higher shear rates, they were smaller and more spherical (Figs. 4B and C).

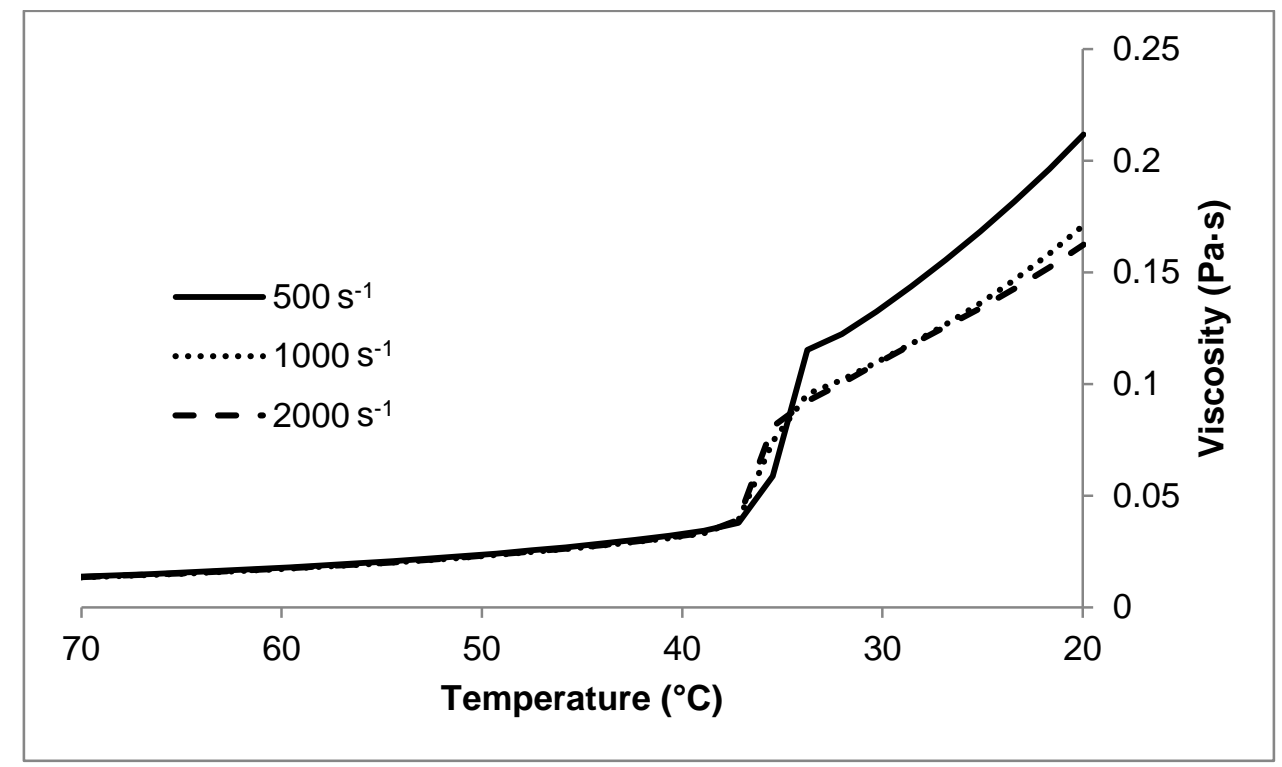

Figure 3. Viscosity profiles of $10 \mathrm{wt} \% \mathrm{FHCO}$ and $90 \mathrm{wt} \% \mathrm{CO}$ blends at a cooling rate of 1.0 ${ }^{\circ} \mathrm{C} / \mathrm{min}$ as a function of shear rate. Error bars removed for clarity $(\mathrm{n}=3)$. 

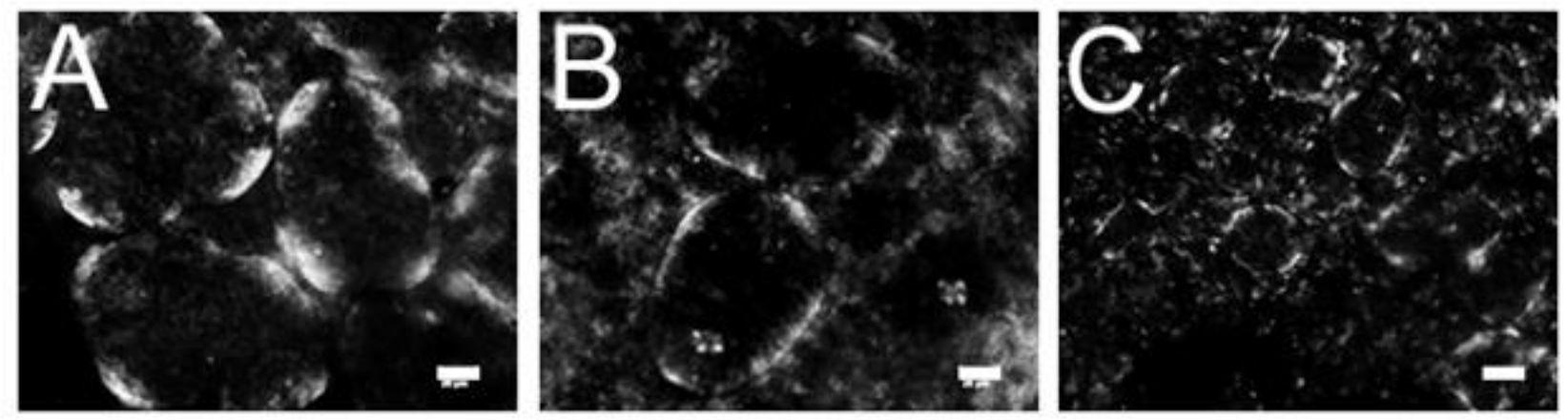

Figure 4. Microstructure of crystallized FHCO as a function of shear rate: A) $500 \mathrm{~s}^{-1}$; B) $1000 \mathrm{~s}^{-1}$; C) $2000 \mathrm{~s}^{-1}$ (cooling rate $=1{ }^{\circ} \mathrm{C} / \mathrm{min}$ and gap size $=0.5 \mathrm{~mm}$ ). Size bar represents $\mu \mathrm{m}$.

The rates of increase in viscosity differed significantly depending on the cooling and shear rates used (Table 2) $(\mathrm{P} \leq 0.05)$. Those cooled at $0.5^{\circ} \mathrm{C} / \mathrm{min}$ showed decreasing rates of viscosity buildup with higher shear rates whereas samples cooled at 1.0 and $1.5{ }^{\circ} \mathrm{C} / \mathrm{min}$ showed significantly higher rates of viscosity increase only for samples sheared at $500 \mathrm{~s}^{-1}$ with no differences between samples sheared at 1000 and $2000 \mathrm{~s}^{-1}(\mathrm{P}>0.05)$. 
Table 2. Rates of increase in viscosity (units of $\mathrm{Pa} \cdot \mathrm{s} /{ }^{\circ} \mathrm{C}$ ) at different cooling and shear rates (results have been multiplied by a factor of $10^{-6}$ for formatting).

\begin{tabular}{ccccc} 
Cooling rate $\left({ }^{\circ} \mathbf{C} / \mathbf{m i n}\right)$ & Shear rate $\left(\mathbf{s}^{-1}\right)$ & Phase I & Phase II & Phase III \\
\hline \hline 0.5 & 500 & 0.06 & 1.50 & 0.46 \\
& 1000 & 0.06 & 1.39 & 0.40 \\
& 2000 & 0.06 & 1.59 & 0.34 \\
\hline 1.0 & 500 & 0.07 & 2.91 & 0.74 \\
& 1000 & 0.07 & 1.72 & 0.50 \\
& 2000 & 0.07 & 2.06 & 0.48 \\
\hline 1.5 & 500 & 0.07 & 2.46 & 0.69 \\
& 1000 & 0.07 & 2.89 & 0.59 \\
& 2000 & 0.07 & 2.69 & 0.55 \\
\hline
\end{tabular}

Size measurements revealed no significant differences in spheroid aspect ratios between samples sheared at 500, 1000, and $2000 \mathrm{~s}^{-1}$ (Table 3) $(\mathrm{P}>0.05)$. However, smaller standard deviations in average long and short axis values with increments in shear rate suggested a narrowing in spheroid size distribution. Overall, it is clear that spheroid size and shape were responsible for the differences in final apparent viscosity, i.e., larger, irregularly-shaped spheroids resulted in higher viscosities than smaller, more-spherical spheroids, as has been previously noted (Acevedo et al., 2012; Kloek et al., 2005; Maleky et al., 2011; Mazzanti et al., 2003).

Table 3. Average length of the long and short axes of the crystal spheroids (cooling rate $=1.0$ $\left.{ }^{\circ} \mathrm{C} / \mathrm{min}\right)$.

\begin{tabular}{cccc} 
Shear rate $\left(\mathbf{s}^{-1}\right)$ & Average long axis $(\boldsymbol{\mu m})$ & Average short axis $(\boldsymbol{\mu m})$ & Aspect ratio \\
\hline \hline 500 & $42.86 \pm 21.2$ & $32.93 \pm 16.6$ & $1.32 \pm 0.2$ \\
1000 & $29.08 \pm 13.1$ & $21.60 \pm 8.0$ & $1.34 \pm 0.2$ \\
2000 & $31.04 \pm 10.2$ & $24.89 \pm 7.9$ & $1.26 \pm 0.2$ \\
\hline
\end{tabular}




\subsection{Effect of a Shear Step}

Whether TAG blends exhibit structuring in the liquid state is debatable, with some studies arguing against (Cebula, McClements, Povey, \& Smith, 1992; Cebula, McClements, \& Povey, 1990; Kellens, Meeussen, Hammersley, \& Reynaers, 1991) and others finding otherwise (Callaghan \& Jolley, 1977; Dibildox-Alvarado et al., 2010; Ueno, Minato, Seto, Amemiya, \& Sato, 1997). To examine whether there existed any liquid-state ordering, samples were subjected to a shear step at $70{ }^{\circ} \mathrm{C}$ before they were cooled and crystallized. The rationale was that if shear could induce measurable ordering or orientation of TAG molecules in the liquid phase, these could act as nucleation sites. The present results showed that pre-shearing did not have any effect on crystal spheroid formation, morphology, or viscosity profile. These results were in contrast to those of De Graef et al. (2009), who found that a shear step had significant effects on palm oil crystallization (De Graef et al., 2009). This was likely related to the extent of pre-shear applied which was lengthier in the work of De Graef et al. (2009). Alternatively, it is likely that pre-shear temperature may be an important contributor worthy of further consideration. At $70{ }^{\circ} \mathrm{C}$, the thermal energy of the individual TAGs may have been too high to permit any sufficiently long shear-modified selfassembly.

\subsection{Mechanism of Formation}

Apparent viscosity and microstructure were characterized as a function of time to elucidate the mechanism of spheroid formation (Figs. 5 and 6). To do so, samples were shear-crystallized for set periods of time until a pre-determined temperature was reached after which viscosity and microstructure were measured. 


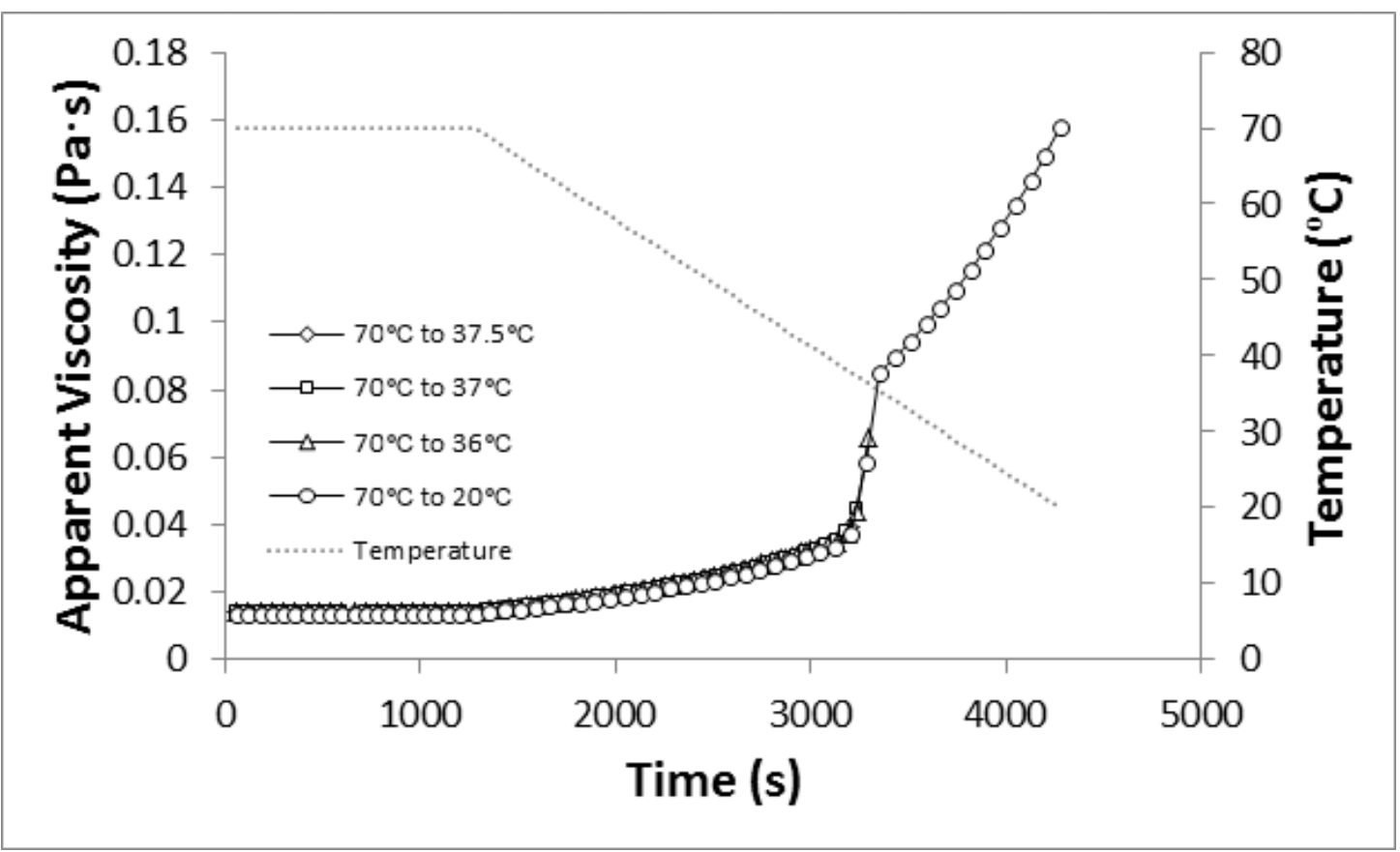

Figure 5. Viscosity profile of FHCO crystallized at $2000 \mathrm{~s}^{-1}$ and $1.0{ }^{\circ} \mathrm{C} / \mathrm{min}(0.5 \mathrm{~mm}$ gap size $)$ as a function of time and temperature $(n=3)$.

Fig. 5 shows the apparent viscosity vs. time curve for FHCO crystallized at $2000 \mathrm{~s}^{-1}$ and

${ }^{\circ} \mathrm{C} / \mathrm{min}$ as a function of time. Crystallization was halted at the various time/temperatures within phase II. There were no significant differences in the apparent viscosity profiles since the only parameter varied was crystallization time. However, there were significant differences in microstructure (Fig. 6). 

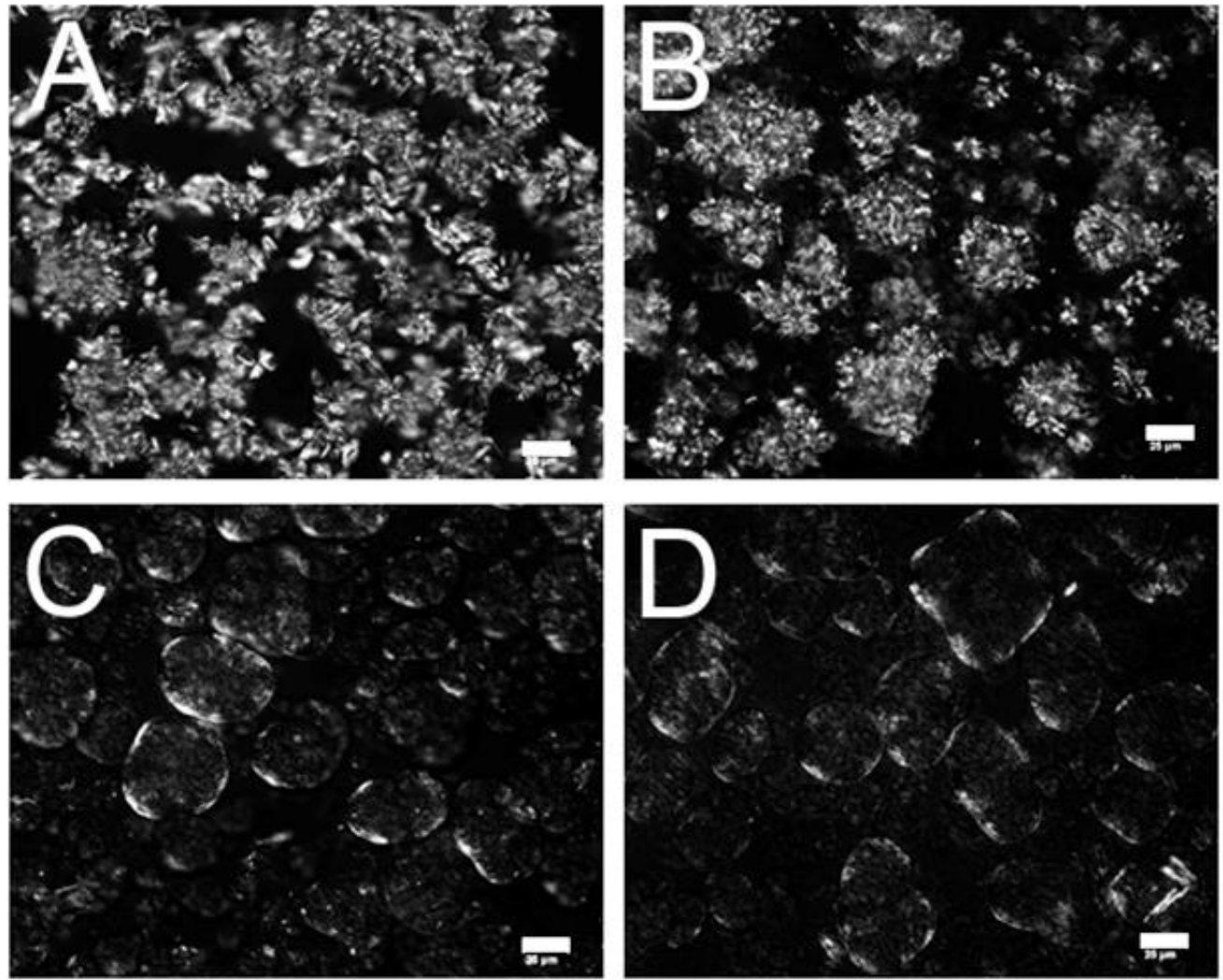

Figure 6. Microstructure of crystallized FHCO under $2000 \mathrm{~s}^{-1}$ shear rate $(0.5 \mathrm{~mm}$ gap size $)$ as a function of crystallization temperature. A) $37.5^{\circ} \mathrm{C}$; B) $\left.37^{\circ} \mathrm{C}\right) 36{ }^{\circ} \mathrm{C}$; D) $20^{\circ} \mathrm{C}$; Size bar represents $25 \mu \mathrm{m}$.

When shear was halted at $37.5{ }^{\circ} \mathrm{C}(\mathrm{t} \approx 3230 \mathrm{~s}$, Fig. 6A), no crystal spheroids were observed. Instead, the crystals had a spherulite-like morphology and were randomly-oriented. Although it appeared that the crystals formed a 3D network, this was not the case as there were no significant differences in sample fluidity or viscosity between these samples and those cooled to lower temperatures. Samples cooled to $37^{\circ} \mathrm{C}(\mathrm{t} \approx 3280 \mathrm{~s})$ showed a higher degree of structuring as evidenced by the spheroidal morphology (Fig. 6B). These rough aggregates were potentially the core for ensuing smooth-surfaced spheroids or a different type of spheroid altogether where crystallization at a higher temperature (i.e., less under-cooling) resulted in larger constituent crystals within the spheroids and therefore a rougher surface. Examining the microstructure of 
samples cooled to $36{ }^{\circ} \mathrm{C}(\mathrm{t} \approx 3300$ s) revealed fully-formed crystal spheroids (Fig. 6C). Interestingly, this time/temperature point was associated with the sharp increase in apparent viscosity in Fig. 5. These images suggested that crystal spheroids may form directly from the melt, with no evidence of precursor structures. Perhaps the action of shear promoted the clustering of $\alpha$ crystallites/nuclei that, under a tumbling action in the shear field, crystallized together as a globular mass. At this temperature, the spheroids had smooth surfaces which may have resulted from either shear-induced heating or viscous heat generation (Eisenmann, Kim, Mattsson, \& Weitz, 2010). In this context, the imposed shear produced enough heat at the spheroid surface to cause melting of less-dense or loosely-associated crystals leaving behind nuclei as templates for the growth of $\beta^{\prime}$ and more likely $\beta$-crystals with their higher density and degree of organization.

Upon visual inspection, there were no distinguishable differences in crystal morphology between samples cooled to $36^{\circ} \mathrm{C}$ or $20^{\circ} \mathrm{C}$ (Figure $6 \mathrm{D}, \mathrm{t} \approx 4250 \mathrm{~s}$ ), implying that once the spheroids have formed, increased shearing time did not impact their morphology. Based on these results, $36-$ $37^{\circ} \mathrm{C}$ was the optimal temperature range for FHCO spheroid formation. To probe this critical zone further, samples were subjected to extra shearing time while being held isothermally at $37^{\circ} \mathrm{C}$ (Fig. 7). 


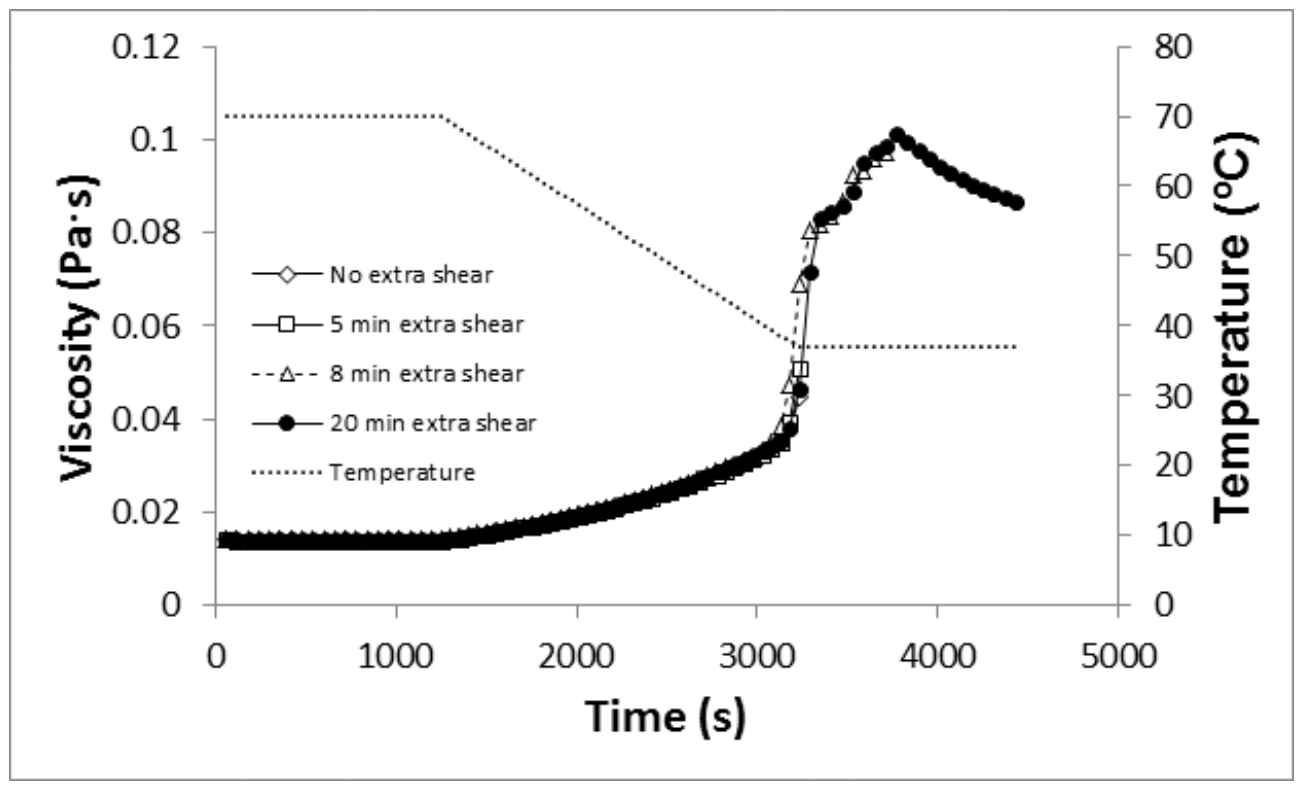

Figure 7. Viscosity profiles of FHCO crystallized at $2000 \mathrm{~s}^{-1}$ at $1.0{ }^{\circ} \mathrm{C} / \mathrm{min}(0.5 \mathrm{~mm}$ gap size $)$ as a function of shearing time.
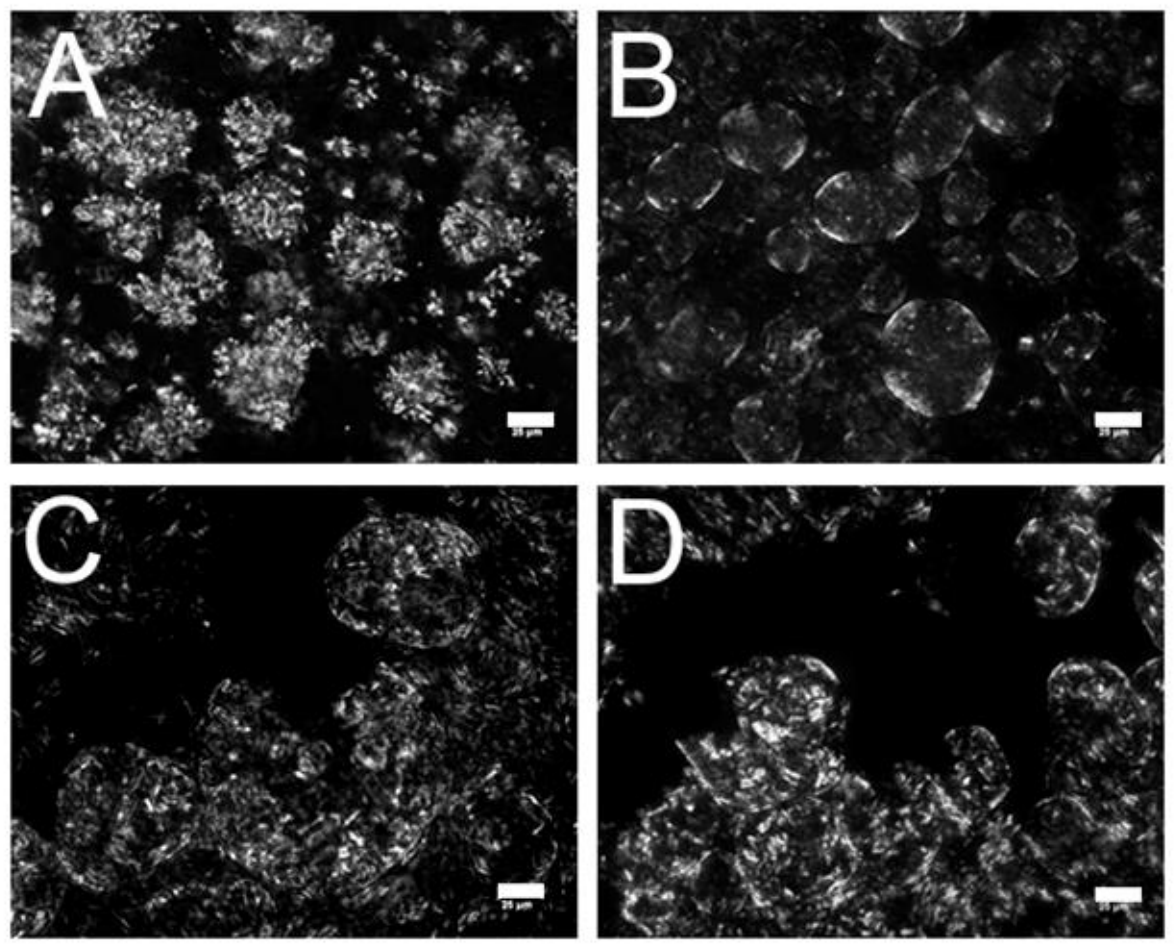

Figure 8. Microstructure of FHCO crystallized at $2000 \mathrm{~s}^{-1}(0.5 \mathrm{~mm}$ gap size $)$ and at $37{ }^{\circ} \mathrm{C}$ as a function of extra shearing time: A) no extra shear; B) $5 \mathrm{~min}$; C) $8 \mathrm{~min}$; D) $20 \mathrm{~min}$. Size bar represents $25 \mu \mathrm{m}$. 
With no extra shear (Fig. 8A), samples showed crystal clusters with a rough globular structure. Samples sheared for an extra 5 min (Fig. 8B) showed fully-formed crystal spheroids with smooth surfaces, which implied that spheroid formation not only depended on shear rate, cooling rate, and crystallization temperature, but also on shearing time. There was a critical value for shearing time as shown by the decrease in viscosity after 8 min of extra shear (Fig. 7) and corresponding breakdown of the spheroids (Fig. 8C). Similar results were visible upon shearing for an extra 20 min, where fractured spheroids surrounded by fragmented crystals dominated (Fig. 8D). Samples crystallized to lower temperatures (e.g., $20^{\circ} \mathrm{C}$, Fig. 6D) did not exhibit this behaviour even though the samples were sheared for longer times, implying that spheroid breakage resulted from the different crystallization temperatures.

With the application of 8 or $20 \mathrm{~min}$ of extra shear vs. $5 \mathrm{~min}$ at $37^{\circ} \mathrm{C}$, the dismembered spheroids demonstrated a higher degree of crystallinity, supporting the transition from $\alpha$ to $\beta^{\prime} / \beta$ crystals. Generally speaking, crystal size increases in the order of $\alpha<\beta^{\prime}<\beta$, with $\alpha$ being characterized as 'wispy', $\beta$ ' more fine-grained, and $\beta$ as larger (AOCS, 1991). Speculatively, evolution from smaller $\alpha$ crystals to larger $\beta^{\prime}$ or $\beta$ crystals in a short time span may be the reason for the structural breakdown observed for the crystal spheroids, i.e., as the spheroids' internal $\alpha$ crystals underwent transformation and growth, their volume increased beyond the initial volume of the spheroids which could no longer contain them leading to breakage.

Significant insights regarding fat crystal spheroid formation may be gleaned from efforts in polymer and colloid research. In polymer systems, the presence of shear may have significant effects on crystal microstructure. Below a critical shear rate, polymers often crystallize into spherulites. Above a critical shear rate, polymer chains may become disentangled and oriented, resulting in crystallization into unique-looking structures such as "shish-kebabs" (Zhang et al., 
2008). These structures then act as a cylindrical core and undergo epitaxial growth, resulting in fibrillar/tubular structures. We postulate that a similar mechanism may have led to spheroid formation. It has been shown that shear forces can affect TAG nucleation, causing nanoplatelets to elongate and orient in the flow direction (Acevedo \& Marangoni, 2014b; Mazzanti et al., 2003). Maleky and Marangoni found that shear caused preferential orientation of TAG crystals in the direction of shear flow but not in the normal plane (Maleky \& Marangoni, 2008). Recent research based on computer simulations to model TAG crystal nanoplatelet aggregation showed that under quiescent conditions, the nanoplatelets aggregated into elongated stacks with "sandwich"-like structures (Marangoni et al., 2012; Peyronel, Ilavsky, Mazzanti, Marangoni, \& Pink, 2013; Pink et al., 2013). These stacks then went on to form random clusters that eventually resulted in a 3D network. We believe that shear would have a significant effect on the aggregation behaviour of these nanoplatelet stacks (Fig. 9A), i.e., below a critical shear rate, the stacks would become oriented in the direction of shear, resulting in greater inter-stack interactions leading to bundle/cylindrical structures (Fig. 9B). These cylindrical structures would then grow lengthwise until they would break along weak points resulting in free-floating cylinders that tumble under shear (Fig. 9C and D). These cylinders could then act as seeds for further epitaxial crystal growth (Fig. 9E). Above a critical shear rate, TAG stacks would tumble faster and have less time to interact, leading to more globular structures and less cylinder formation (Fig. 9F). These crystallite aggregates may then act as seeds for further epitaxial growth into spheroids (Fig. 9G). 


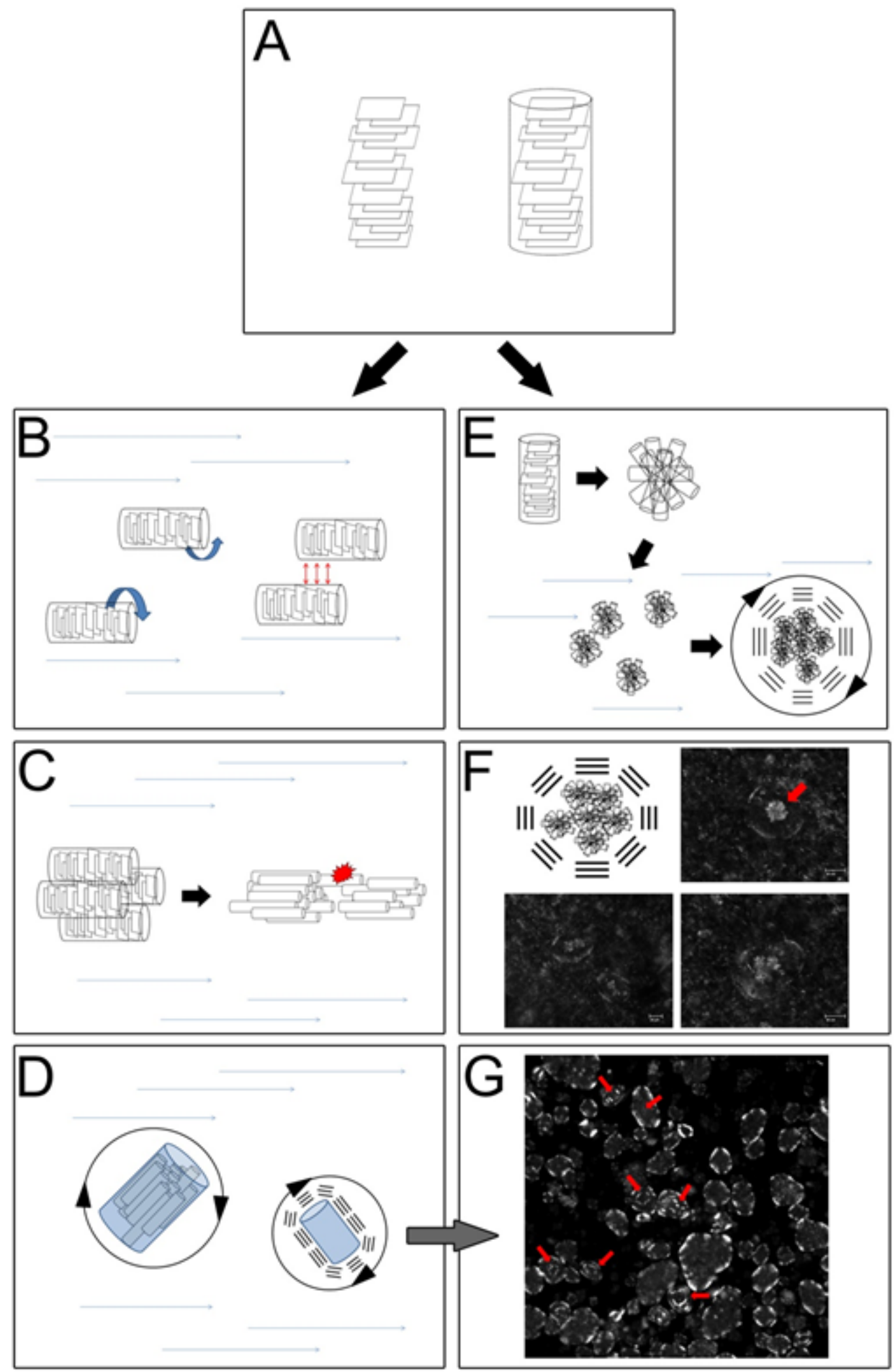

Figure 9. Proposed mechanism for the formation of crystal spheroids. 
The proposed mechanism of crystal spheroid formation is thus multi-step: i) potential shearinduced pre-structuring of TAGs in the melt; ii) nucleation and formation of nanoplatelets (relatively large distances between nanoplatelets and thus low degree of inter-particle interactions); iii) rotation and tumbling of platelets due to shear in the flow field; iv) nanoplatelet aggregation into extended stack-like structures; v) clustering of the nanoplatelet stacks into globular aggregates or bundles, depending on the shear rate; vi) crystallization into clusters of $\alpha$ crystals while tumbling, forming crystal spheroids; vi) shear acceleration of the $\alpha \rightarrow \beta^{\prime}$ (or $\beta$ ) polymorphic transition and finally vii) viscous heat generation causing melting of loosely-associated $\alpha$ crystals at the spheroid surface, leaving behind nuclei that act as templates for the formation of moreorganized $\beta^{\prime}$ (or $\beta$ ) crystals, resulting in smoother surfaces. Tarabukina et al., observing the crystallization of palm oil under shear via optical microscopy and small-angle light scattering, found that palm oil crystallized in a similar stepwise fashion: i) nanoscopic crystallites formed needle-like crystals; ii) needle clustering to form spherulites; and iii) aggregation of the spherulites to form large masses with globular domains or networks (Tarabukina et al., 2009). Chen and Doi modeled the interaction of hard spheres in the presence of shear and found that over time, the spheres would aggregate into globular clusters (Doi \& Chen, 1989). It was also found that the viscosity profiles of the sheared hard spheres were sigmoidal in shape (first increasing slowly, then steeply, and finally leveling off) - much like the viscosity profiles observed for the formation of the present crystal spheroids (Fig. 1). They also observed that viscosity decreased with increasing shear rate, correlating well with our data (Fig. 3).

Classical nucleation theory (CNT) models the free energy requirements for nucleation and crystal growth. While the crystalline phase is usually more stable and favoured energetically, there is usually an energy barrier that suppresses formation of smaller nuclei below a critical nucleus size 
(Lander, Seifert, \& Speck, 2013). Subjecting a colloidal system to shear flow drives the system out of thermal equilibrium which changes the concept of free energy and renders the CNT inapplicable. Sheared colloidal suspensions may still crystallize, but with nucleation and growth kinetics that differ significantly from those under static conditions. In a numerical study on crystallization in a supersaturated suspension of repulsive colloidal particles, Lander et al. found that shear suppressed nuclei formation via disruption of pre-nucleation-structuring in the melt but enhanced growth once a critical nucleus had formed (Lander et al., 2013). This suggested the possibility of competing mechanisms in regards to the nucleation and growth under shear. In this light, the application of shear likely altered TAG nucleation and growth kinetics of systems in an incipient state of crystallization.

Cerda et al. studied the effects of shear on crystal nucleation in colloidal suspensions via twodimensional Langevin dynamics simulations (Cerdà, Sintes, Holm, Sorensen, \& Chakrabarti, 2008). Though colloid self-assembly clearly differs from that of TAGs, this study showed that shear may exert mechanical stress that either pulls particles apart, weakening effective attractive interactions between them or enhances the rate of collisions between particles, increasing the probability of forming stable nuclei. Shear promoted compaction of crystal clusters, whereby cluster size increased until a critical value above which it caused their breakdown. As well, at high shear rates, surface erosion of smaller particles limited cluster size. Finally, shear in combination with friction forces mediated cluster rotation, with the kinetic energy gained via shear-flow tumbling responsible for eventual breaking of the cluster into smaller fragments.

In summary, the crystallization of TAGs is still not fully understood let alone within a shear field. Using other model systems may help to further elucidate mechanisms where it has been shown 
that shear modulates particle-particle interactions and ensuing morphology via selection of different processing conditions.

\subsection{Crystal Spheroid Stability}

The initial structure of the crystal spheroids (Fig. 10A) became more crystalline internally with time, which was accompanied by crystal growth in the continuous phase (Fig. 10B). As the spheroids aged, they broke down (Fig. 10C) and resembled samples sheared isothermally (e.g., Figs. 8C and D), corroborating that shear accelerated polymorphic transitions and crystal growth.
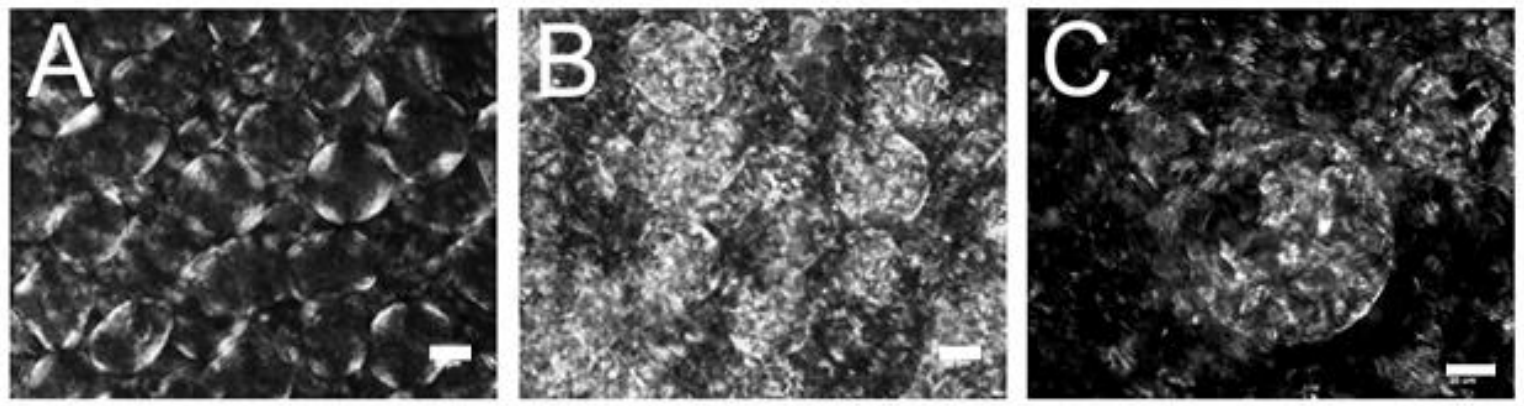

Figure 10. Evolution of crystal ovoid microstructure over time $\left(2000 \mathrm{~s}^{-1}, 1.0^{\circ} \mathrm{C} / \mathrm{min}, 0.5 \mathrm{~mm}\right.$ gap $)$ : A) Day 0; B) Day 7; C) Day 7 at 630 X magnification. Bar in A and B represents $100 \mu \mathrm{m}$. Bar in $\mathrm{C}$ represents $20 \mu \mathrm{m}$.

Fig. 11 shows the microstructure of samples taken immediately after formation (day 0 ) and after 7 days of storage. On day 0 , those sheared at $500 \mathrm{~s}^{-1}$ showed the lowest crystallinity in the continuous phase whereas those sheared at $2000 \mathrm{~s}^{-1}$ showed the highest proportion. This indicated that a higher proportion of FHCO was incorporated into the spheroidal structures at lower shear rates. Higher shear rates may have inhibited the interaction between TAG nanocrystal clusters, resulting in an overall reduction in the breadth of the spheroid size distribution but an increase in the number of crystals in the continuous phase. Overall, the spheroids became more crystalline 
over time and the number of continuous phase crystals increased but these did not increase in size. Hence, the proportion of FHCO that crystallized into spheroids $v s$. bulk crystals affected sample viscosity and rheology over time.
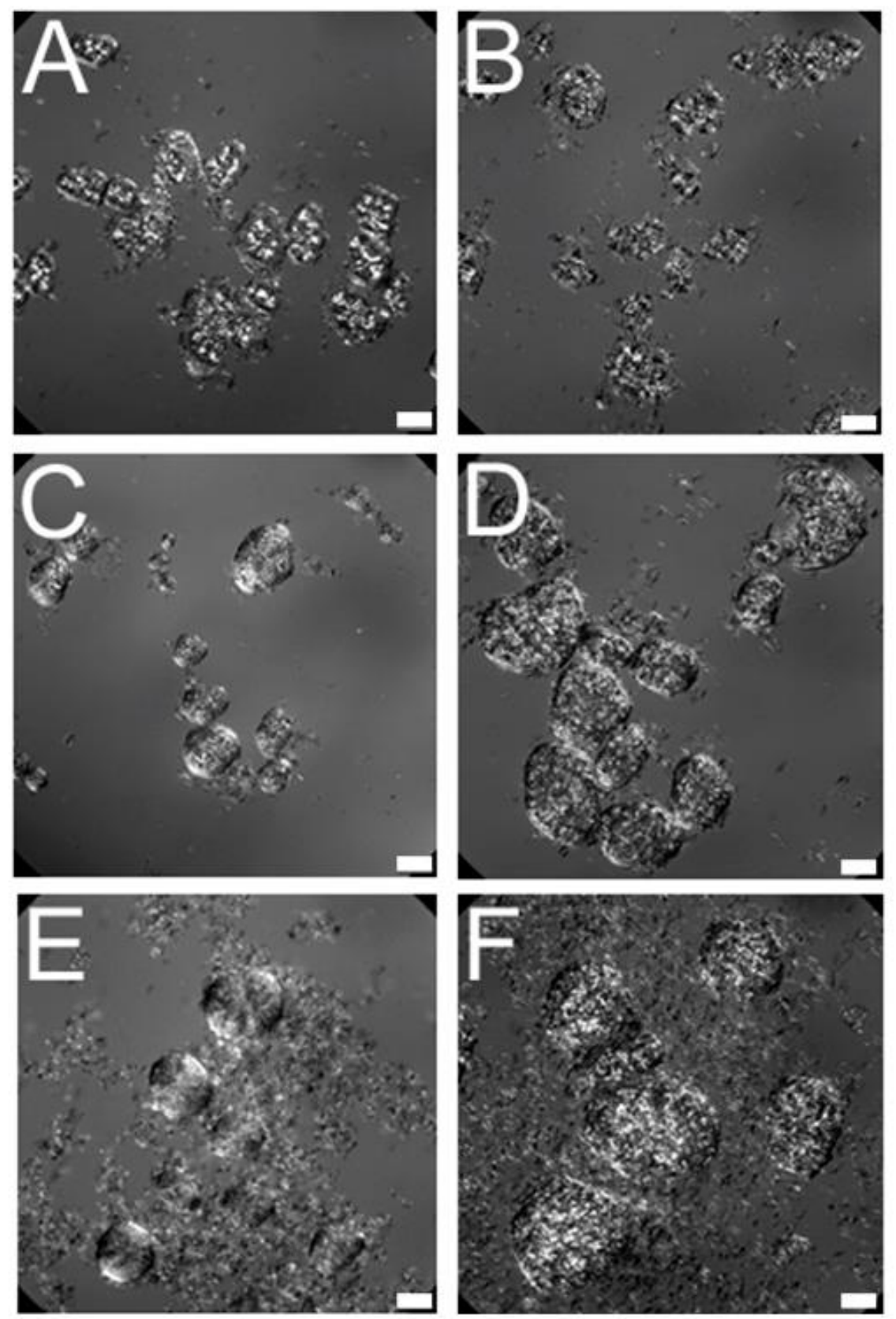

Figure 11. Partially-polarized images of crystal growth from day 0 [A) $500 \mathrm{~s}^{-1}$; C) $1000 \mathrm{~s}^{-1}$; E) $2000 \mathrm{~s}^{-1}$ ] to day 7 [B) $500 \mathrm{~s}^{-1}$; D) $1000 \mathrm{~s}^{-1}$; F) $2000 \mathrm{~s}^{-1}$. Size bar represents $30 \mu \mathrm{m}$. 


\section{Conclusion}

We explored the formation of spheroidal fat crystal assemblies and elaborated on existing mechanisms to develop a new model possibly responsible for their formation. This work demonstrated that processing conditions can have a profound impact on the crystallization of a model fat blend. Both cooling and shearing were required for the formation of crystal spheroids with the latter having a greater impact. Optimized processing conditions resulted in spheroids with a smoothened exterior and minimal internal crystallization. With age, the spheroids eventually broke down due to the continued growth of internal crystals. Future investigations are underway to further characterize spheroid stability and the fat crystal polymorphs present upon spheroid formation during ageing. Overall, the present study has shown that it is possible to tailor fat crystal aggregation and possibly rheological behaviour. 


\section{Chapter 3 - Spheroidal Fat Crystals: Structure Modification via use of Emulsifiers}
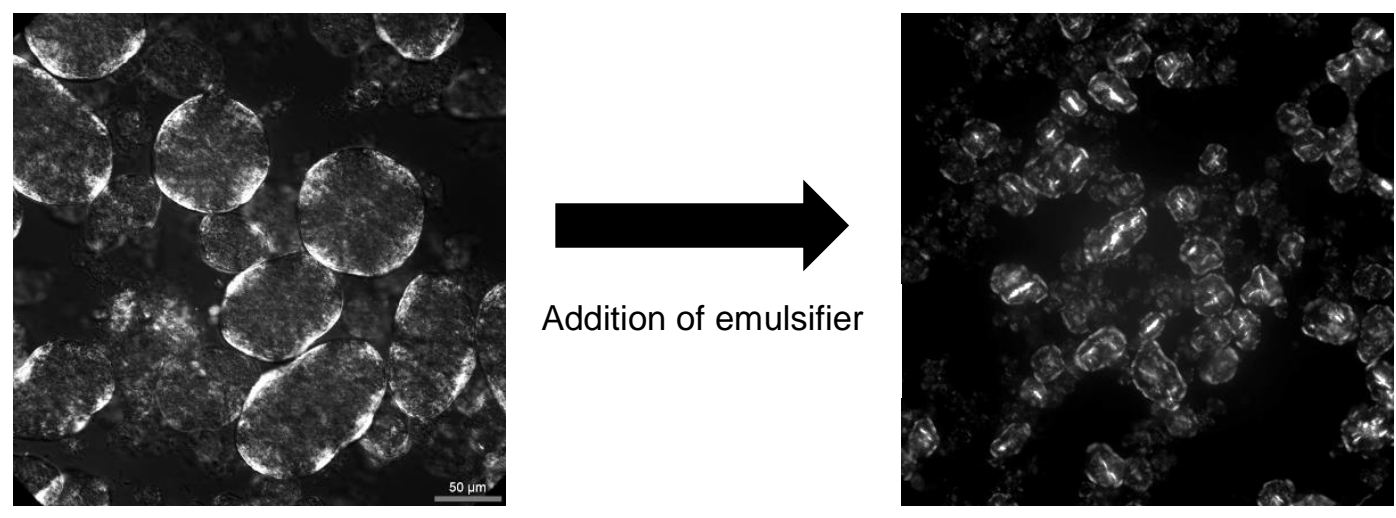

- We examine the effect of emulsifiers on the shear-crystallization of model fat systems

- The type and concentration of emulsifier significantly affects fat crystal morphology

- Shear affects fat polymorphism

- Shear affects emulsifier incorporation into fat crystals 


\section{Abstract}

Emulsifiers were used to modify the kinetics of formation and morphology of spheroidal fat crystal assemblies generated via the confined gap shear-cooling of model fat systems consisting of fullyhydrogenated canola oil (FHCO), canola oil (CO), and glycerol monostearate (GMS), glycerol monopalmitate (GMP), sorbitan monostearate (SMS), or sorbitan tristearate (STS). The presence and morphology of spheroidal fat crystals were dependent on emulsifier type and concentration, with GMS, GMP and SMS significantly reducing spheroid size at lower concentrations and causing deformation of the crystals into irregular and elongated shapes at higher concentrations. STS significantly hindered the formation of spheroidal crystals and instead promoted globular crystalline assemblies. Thermal analysis and X-ray diffraction revealed that samples crystallized primarily into the $\beta^{\prime}$ polymorph along with a smaller amount of $\alpha$ polymorph. The ratio of $\alpha$ to $\beta^{\prime}$ polymorphs generally increased with emulsifier concentration and imposition of higher laminar shear rates, suggesting the importance of two phenomena: i) shear may influence the interactions between triacylglycerols at a molecular level, affecting the type of nanoplatelets formed and their interactions to form crystallites and subsequent crystals, and ii) the extent of shear (rate/duration) may affect the rate and/or amount of emulsifier incorporation into triacylglycerol crystal lattices. Overall, these results demonstrated that fat crystal structures may be tailored with the combined use of emulsifiers and laminar shear. 


\section{Introduction}

Many characteristics of fat-containing processed food products, including their mechanical properties (e.g. firmness and spreadability), eating properties (e.g. mouthfeel or meltability), physical stability (e.g. phase separation) and visual appearance depend on the composition of the fat phase [i.e. triacylglycerol (TAG) make up, presence of emulsifiers and minor components, etc.] and processing conditions (e.g., cooling rate, crystallization temperature, time and shear). Together, these parameters affect the rate and extent of TAG crystallization and polymorphic behaviour as well as aggregation into 3D fat crystal networks ultimately responsible for product functionality (Peyronel, Pink, \& Marangoni, 2014; Sato, 2001; Walstra, 2003).

The use of emulsifiers and shear-cooling are two means to modify fat crystal structure and kinetic stability. Emulsifiers are surface-active compounds that consist of hydrophilic and hydrophobic moieties, with the relative size, volume fraction, and composition of each dictating whether the molecule will be oil or water-tending. The focus of this research was on oil-tending emulsifiers that, as previously shown, may prevent or promote TAG nucleation, crystal growth, and/or polymorphic transitions, depending on their type, concentration, molecular similarity with the crystallizing TAG species and mode of action (Acevedo \& Marangoni, 2014a; Aronhime, Sarig, \& Garti, 1990; Shimamura, Ueno, Miyamoto, \& Sato, 2013; Smith, Bhaggan, Talbot, \& van Malssen, 2011). By manipulating processing conditions such as mixing, fat crystal morphology and aggregation behaviour can be extensively tailored, resulting in products with very different textures. Additionally, shear-cooling plays a significant role on the fat crystal morphology and polymorphism obtained during a prescribed cooling regime (Campos et al., 2002; Herrera \& Hartel, 2000; Rousseau et al., 2005). Faster cooling typically leads to less stable structures (e.g., the $\alpha$ or $\beta^{\prime}$ form vs. the $\beta$ with slower cooling) whereas imposition of shear usually promotes fat 
crystal aggregation into larger microstructures and accelerates polymorphic transitions towards higher stability form(s) (e.g., the $\beta^{\prime}$ or $\beta$-forms vs. the $\alpha$ form) (MacMillan $\&$ Roberts, 2002; Maleky \& Marangoni, 2008; Mazzanti et al., 2003).

The last few years have seen the exploration of laminar shear as a tool to induce TAG crystal orientation and alignment at the nanoscale (Maleky \& Marangoni, 2011; Maleky et al., 2011) whereas at the microscale, such confined gap shear may produce novel ovoid-shaped crystals due to a tumbling action (Acevedo et al., 2012; Tran et al., 2014). In this study, we expand on previous work studying the formation of spheroidal fat crystals and examine the effects of different emulsifiers on the formation and microstructure of fat crystal spheroids. Four emulsifiers were used to alter spheroid formation: glycerol monostearate (GMS) and monopalmitate (GMP) to evaluate the effect of monoacylglycerol (MAG) chain length and sorbitan monostearate (SMS) and tristearate (STS) to compare the impact of polar group size (i.e., sorbitan vs. glycerol). Though strong evidence has shown the impact of such emulsifiers on bulk fat crystallization (Verstringe, Danthine, Blecker, Depypere, \& Dewettinck, 2013; Verstringe, Danthine, Blecker, \& Dewettinck, 2014; Verstringe, Dewettinck, Ueno, \& Sato, 2014), their impact on spheroid formation has never been reported. 


\section{Materials and Methods}

\subsection{Materials}

Canola oil $(\mathrm{CO})$ (acid value $\sim 0.2 \%$ ) was purchased from a local grocery store and stored at room temperature. Fully-hydrogenated canola oil (FHCO) was purchased from Bunge (Oakville, ON, Canada) and had a capillary melting point of $69.5^{\circ} \mathrm{C}$ (AOCS official method Cc 1-25) and a free fatty acid content of $0.018 \%$ (AOCS Society, 1998). The predominant TAG species in FHCO were $\mathrm{C}_{52}(13.3 \%), \mathrm{C}_{54}(73.6 \%)$, and $\mathrm{C}_{56}(5.0 \%)$. Distilled glycerol monostearate (Alphadim $90 \mathrm{SBK}$ ) and glycerol monopalmitate (DIMODAN® HP K-A) were obtained from DANISCO USA INC. (Madison, WI, USA). Distilled sorbitan monostearate (Span 60) and sorbitan tristearate (Span 65) were purchased from Sigma-Aldrich (Saint Louis, MO, USA). All components were used as received from suppliers without further purification.

\subsection{Shear-Crystallization}

FHCO and emulsifiers were mixed to give a $10 \mathrm{wt} \%$ solution in $\mathrm{CO}$ and heated to $70{ }^{\circ} \mathrm{C}$ with stirring to melt the FHCO and emulsifiers. Aliquots were withdrawn and deposited between the parallel plates (PP25, $25 \mathrm{~mm}$ diameter, $0.5 \mathrm{~mm}$ gap) of a rheometer (Physica MCR301, Anton Paar, Ville St-Laurent, QC, Canada). Samples were kept at $70{ }^{\circ} \mathrm{C}$ for $20 \mathrm{~min}$ and then cooled at $1.0^{\circ} \mathrm{C} / \mathrm{min}$ while being sheared at 500,1000 or $2000 \mathrm{~s}^{-1}$. Apparent viscosity was measured as the samples were sheared and cooled. All experiments were performed at least in triplicate.

\subsection{Microscopy}

Microstructure was observed via polarized light microscopy (PLM). Immediately upon completion of shear-crystallization, samples were placed on viewing slides at room temperature $\left(\sim 25^{\circ} \mathrm{C}\right)$ (Fisher Scientific, Ottawa, ON, Canada), covered with a cover slip (Fisher Scientific, Nepean, ON, Canada) and analyzed with an inverted light microscope (Axiovert 200M, Zeiss Inc., Toronto, ON, Canada) equipped with a CCD camera (QImaging, Inc., RETIGA 4000R, Surrey, BC, Canada) 
using QImaging Q-Capture Pro 7 software (Version 7.0.5 Build 4325, QImaging, Inc., Surrey, BC, Canada).

\subsection{Differential Scanning Calorimetry}

Upon completion of shear-crystallization, approximately 7-10 $\mathrm{mg}$ of sample was sealed hermetically in aluminum pans (PerkinElmer, Woodbridge, ON, Canada) and analyzed using a differential scanning calorimeter (DSC) (Pyris Diamond DSC, PerkinElmer, Woodbridge, ON, Canada). An empty aluminum pan was used as reference and the DSC was calibrated using an Indium standard. Samples were held isothermally at $20{ }^{\circ} \mathrm{C}$ for $5 \mathrm{~min}$ and then heated to $90{ }^{\circ} \mathrm{C}$ at $10{ }^{\circ} \mathrm{C} / \mathrm{min}$. Thermograms were analyzed using Pyris Diamond DSC software (v.7, PerkinElmer, Woodbridge, ON, Canada).

\subsection{X-ray Diffraction}

Small (SAXD) and wide-angle (WAXD) X-ray diffraction of the fats was performed on a Hecus

S3-MICROcaliX (Hecus X-ray Systems GmbH, Graz, Austria). The unit uses a low-power $\quad$ (50 W), high brilliance GeniX microfocus source and customized FOX-3D multilayer point focusing optics (Xenocs SA, Grenoble, France). The X-ray beam was generated by a $50 \mathrm{kV}, \quad 1 \mathrm{~mA} \mathrm{Cu}$ $\mathrm{K} \alpha$ anode. The spectra were captured using Hecus 1-D position-sensitive detectors (model PSD50M). An integrated calorimeter (Setaram Instrumentation, Caluire, France) allowed for in situ crystallization and melting of static samples. Approximately $20 \mu \mathrm{L}$ of melted sample were placed within 1.0 mm O.D. quartz capillary tubes (Charles Supper Company, Inc., Natick, MA, USA) using a $90 \mathrm{~mm}$ needle and syringe and characterized at room temperature. Sheared samples were analyzed at $25^{\circ} \mathrm{C}$ immediately following crystallization. Statically-cooled samples were subjected to a similar cooling protocol as sheared samples: held at $90^{\circ} \mathrm{C}$ for $15 \mathrm{~min}$, then cooled to $10^{\circ} \mathrm{C}$ at 
$1{ }^{\circ} \mathrm{C} / \mathrm{min}$. Scans were collected each minute to capture the polymorphic form of the evolving system.

\subsection{Statistical Analyses}

All numerical data are reported as mean \pm standard deviation (SD) and were analyzed by one-way ANOVA. $\mathrm{P}<0.05$ was considered significant. 


\section{Results and Discussion}

\subsection{Viscosity Profiles}

The presence of emulsifiers had a significant impact on viscosity profiles (Fig. 1). At 0.5 and 1.5 wt $\%$ emulsifier concentrations, the viscosity profiles generally followed the three-phase sigmoidal pattern seen in control FHCO-CO blends (Tran et al., 2014). At low emulsifier concentrations, the first phase saw slight increases in viscosity due to increased interaction between TAG molecules as the temperature was reduced (Dibildox-Alvarado et al., 2010; Tarabukina et al., 2009). The second phase, marked by a sharp increase in viscosity, was attributed to the initial crystallization of the fat (FHCO) into $\alpha$ crystals based on work by De Graef et al., who studied the crystallization of palm oil under constant shear via time-resolved x-ray analysis (De Graef et al., 2009) and work by Mazzanti et al. (Mazzanti et al., 2005). The third phase was attributed to continued crystallization of FHCO directly from the melt as well as $\alpha \rightarrow \beta^{\prime}$ or $\beta$ polymorphic transitions (De Graef et al., 2009; Mazzanti et al., 2005). 


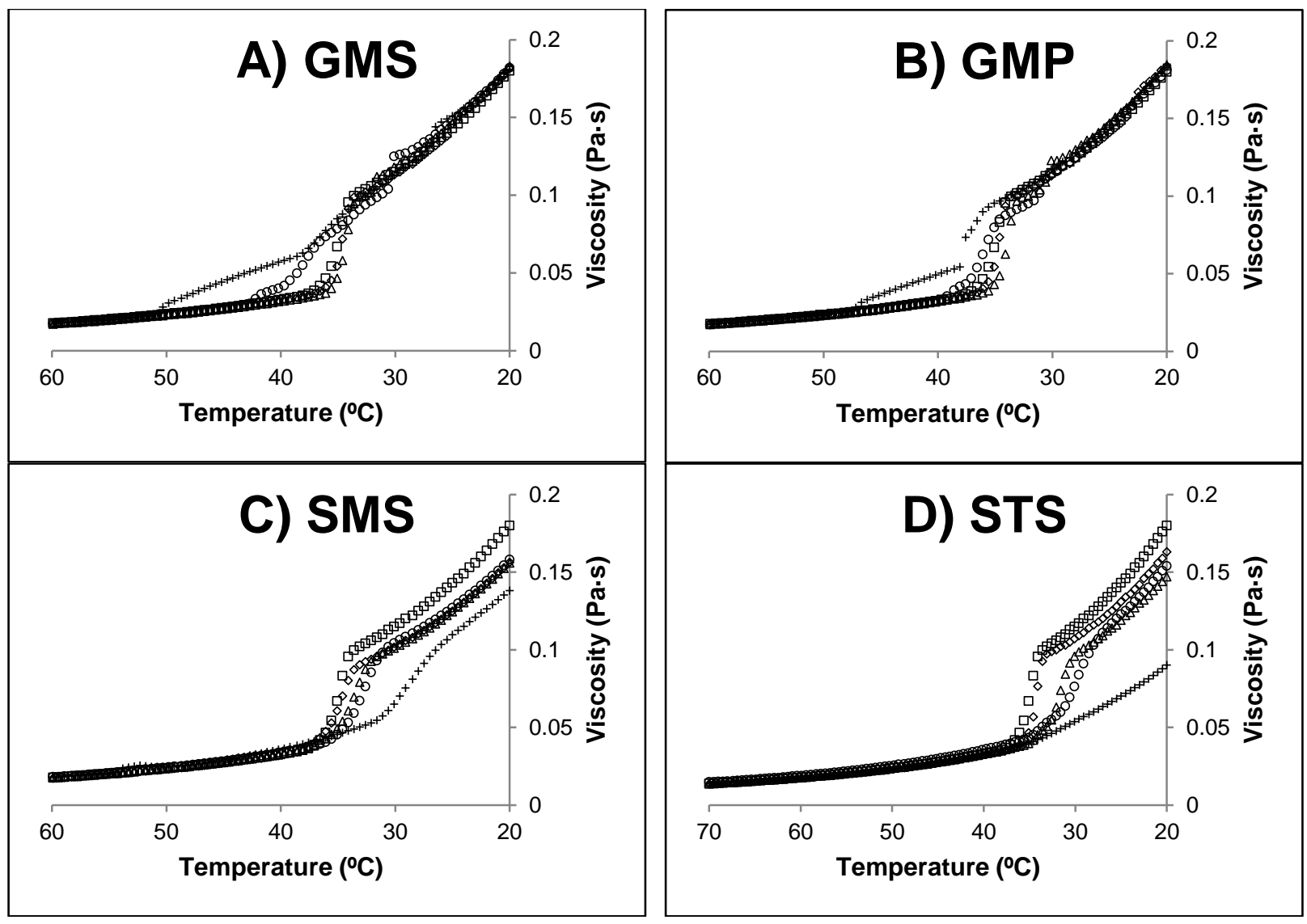

Figure 1. Viscosity profiles of samples sheared at $1000 \mathrm{~s}^{-1}$ : A) GMS; B) GMP; C) SMS; D) STS at varying concentrations: $\square)$ Control $(0 \mathrm{wt} \%) ; \diamond) 0.5 \mathrm{wt} \% ; \triangle) 1.5 \mathrm{wt} \%$; ○) $2.5 \mathrm{wt} \%$; +) $5.0 \mathrm{wt} \%$. Error bars removed for clarity $(n=3)$.

At $0.5 \mathrm{wt} \%$, GMS and GMP showed similar rates of viscosity increase compared to the control although they delayed crystallization to slightly later times and lower temperatures. In comparison, SMS did not delay crystallization but did decrease the rate of viscosity increase while STS showed a delay in crystallization but similar rates of viscosity increase compared to the control. At 1.5 wt $\%$, GMS and GMP showed a continued trend of slightly delaying crystallization while not affecting the rates of viscosity increase whereas SMS and STS showed decreased crystallization temperatures as well as rates of viscosity increase. At $2.5 \mathrm{wt} \%$ emulsifier, GMS and GMP showed higher crystallization temperatures compared to the control. Viscosity profiles began to deviate 
from the three-phase regime described previously with crystallization now appearing to occur in an increased number of steps. This resulted from the MAG concentration crossing a critical threshold above which the crystal lattice of FHCO became saturated with emulsifier and the MAG independently crystallized. This trend continued at 5.0 wt\% MAG, with GMS crystallizing at higher temperatures while showing reduced rates of crystallization (Fig. 1A crosses) and even less distinction between the different phases in the crystallization regime. This was attributed to GMS having a higher degree of molecular similarity to the tristearin in FHCO compared to GMP with its 16 carbon chain. Aronhime et al. showed that for selected emulsifiers, $10 \mathrm{wt} \%$ could be incorporated into the crystal lattice of tristearin without affecting its polymorphic structure (Aronhime, Sarig, \& Garti, 1988). The present results showed effects at 2.5 and $5.0 \mathrm{wt} \%$ (25 and $50 \mathrm{wt} \%$ relative to $\mathrm{FHCO}$ ), suggesting that up to $15 \mathrm{wt} \%$ GMS or GMP could be incorporated into the crystal lattice of FHCO without significantly affecting its structure or viscosity profile. At $\leq$ $1.5 \mathrm{wt} \%$, the presence of GMS and GMP initially decreased the primary crystallization temperatures $(\mathrm{P}<0.05)$ (Table 1$)$. This suggested incorporation of the emulsifiers within the FHCO crystal lattice and slight suppression of crystal seed formation or lattice organization. However, at $\geq 2.5 \mathrm{wt} \%$, both emulsifiers significantly increased the crystallization onset temperature, with the GMS increasing this temperature more so than the GMP $(\mathrm{P}<0.05)$. Beyond the mere presence of more material available for crystallization, this further suggested that the FHCO and MAG were independently crystallizing. Studying the effects of different polyglycerine fatty acid ester (PGFE) emulsifiers on the crystallization of palm stearin (PS), Shimamura et al. showed that below a critical concentration, the PGFEs inhibited crystallization of PS while above it, crystallization was enhanced via templating effects (Shimamura et al., 2013). 
Table 1. Primary crystallization temperatures $\left({ }^{\circ} \mathrm{C}\right)$ of various emulsifier-FHCO-CO blends cooled at $1{ }^{\circ} \mathrm{C} / \mathrm{min}$ while being sheared at $1000 \mathrm{~s}^{-1}(\mathrm{n}=3)$. All standard deviations $=0$.

\begin{tabular}{ccccc}
\hline Concentration & $\mathbf{0 . 5} \mathbf{w t} \%$ & $\mathbf{1 . 5} \mathbf{w t} \%$ & $\mathbf{2 . 5} \mathbf{w t} \%$ & $\mathbf{5 . 0} \mathbf{w t} \%$ \\
\hline GMS & 37.6 & 36.6 & 43.2 & 51.8 \\
GMP & 36.6 & 36.1 & 40.2 & 47.2 \\
SMS & 38.6 & 38.1 & 36.6 & 54.8 \\
STS & 36.6 & 35.6 & 35.6 & - \\
Control & 38.1 & & & \\
\hline
\end{tabular}

Different crystallization and viscosity trends were apparent with SMS and STS vs. the MAGs in large part due to the different polar group (i.e., sorbitan vs. glycerol, respectively). Crystallization onset temperatures and the rates at which viscosity increased were reduced with $\leq 2.5 \mathrm{wt} \% \mathrm{SMS}$ and STS $(\mathrm{P}<0.05)$ (Table 1). As per the MAGs, this suggested incorporation of the emulsifiers within the FHCO crystal lattice and slight suppression of crystal seed formation or lattice organization, which we attributed to the sorbitan moiety's ability to prevent molecular rearrangements via hydrogen bonding between its hydroxyl groups and the glycerol groups on neighbouring tristearin molecules (Aronhime et al., 1988). This was most apparent with STS, where the rates of viscosity increase were reduced with emulsifier concentration and the threephase crystallization regime disappeared completely at $5.0 \mathrm{wt} \%$, suggesting inhibition of the $\quad \alpha$ $\rightarrow \beta^{\prime} / \beta$ transition and the inability of the $\beta^{\prime} / \beta$ crystals to form directly from the melt. However, presence of $5 \mathrm{wt} \%$ SMS resulted in a crystallization event at $\sim 55^{\circ} \mathrm{C}$, suggesting independent crystallization of SMS first followed by FHCO at $\sim 37^{\circ} \mathrm{C}$. 
Generally speaking, final viscosity values were not significantly affected by emulsifier concentration for GMS and GMP, which had similar values to those of the control (Fig. 1 squares), suggesting similar degrees of crystallization and solid fat contents (SFC). Thus, the 2-carbon difference in fatty acid chain length had no significant effects. This is in agreement with work by Wright et al., who found that minor components did not affect the equilibrium SFC of crystallized milk fat (Wright, Hartel, Narine, \& Marangoni, 2000). By contrast, samples with SMS and STS had lower final viscosity values than the control, perhaps due to their ability to retard the $\alpha \rightarrow \beta^{\prime} / \beta$ transition of tristearin (Elisabettini, Desmedt, \& Durant, 1996).

\subsection{Microscopy}

The control consisting of FHCO and CO displayed now-familiar spheroidal crystalline assemblies (Fig. 2) (Tran et al., 2014). The microstructure of the sheared crystals was significantly affected by emulsifier type and concentration (Fig. 3). Compared to the control, samples with $0.5 \mathrm{wt} \%$ glycerol-based emulsifiers (GMS and GMP) consisted of similar, smaller spheroidal structures with larger irregularly-shaped crystals also visible (Fig. 3A and E). The emulsifier-containing spheroids had visible globular crystal cores surrounded by near-transparent shells whereas the

control spheroids were more uniform in structure, lacked distinct crystalline cores and usually displayed Maltese cross patterns. At $1.5 \mathrm{wt} \%$, the average crystal spheroid size decreased abruptly and became more uniform in size (Fig. 3B and F), where the spheroids displayed distinct crystalline cores surrounded by less-dense shells. This, along with corroborating DSC data, indicated that the emulsifiers nucleated before FHCO and acted as seeds for crystal growth and spheroid formation (Basso et al., 2010). With $2.5 \mathrm{wt} \%$ GMS or GMP, there was less distinction between the crystal cores and outer shells and the size distribution broadened with some crystals elongating into sausage-like shapes while others were larger in size, globular, and had jagged 
surfaces (Fig. 3C and G). Samples with GMS contained a greater number of elongated structures compared to GMP-based structures that were more ovoid-shaped. This result indicated that the shape of the core (e.g., needle-like with GMS vs. ovoidal with GMP) played a significant role on the shape of the surrounding spheroid, which adopted the form of the central structure. At $5.0 \mathrm{wt} \%$ GMS, there was increased presence of elongated crystals and spheroids with rough surfaces, likely due to the concurrent crystallization and association of the MAGs and FHCO, resulting in different crystal types that annealed under the action of shear-cooling (Fig. 3D and $\mathrm{H}$ ). The elongated crystals displayed a high degree of crystallinity while the globular roughened spheroids were lesscrystalline compared to the control and were almost transparent.

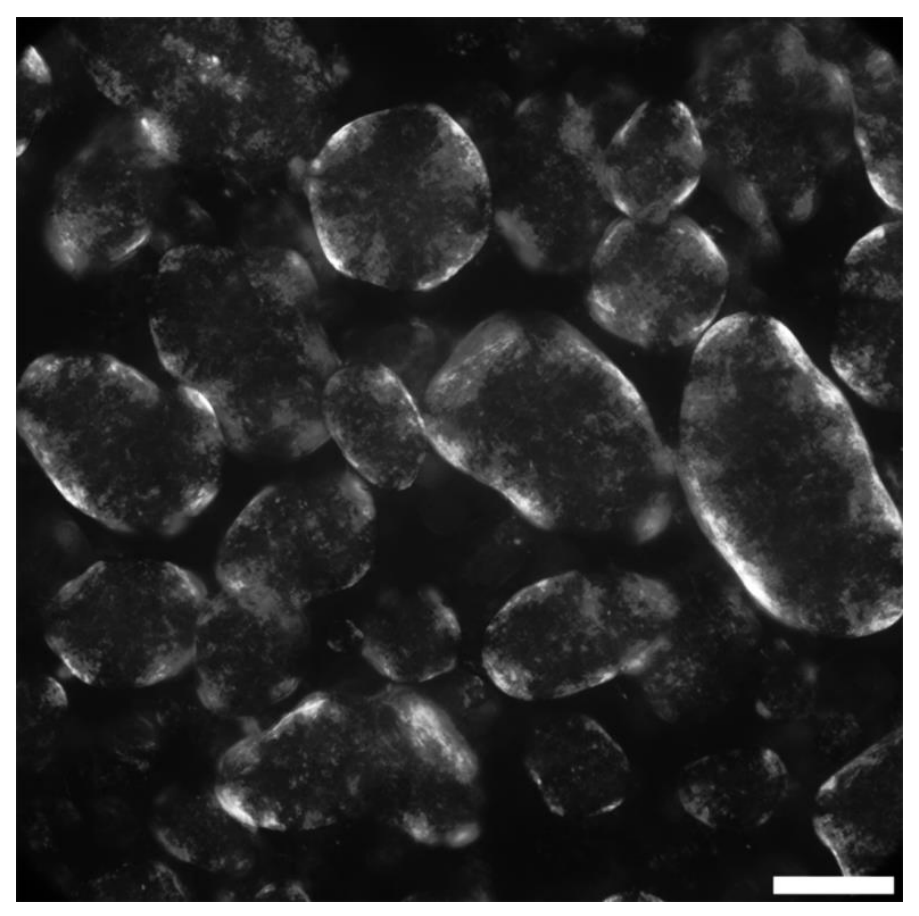

Figure 2. Crystal spheroids formed via shear-crystallization of a $10 \mathrm{wt} \% \mathrm{FHCO}$ and $90 \mathrm{wt} \% \mathrm{CO}$ blend at a cooling rate of $1.0^{\circ} \mathrm{C} / \mathrm{min}$ and a shear rate of $1000 \mathrm{~s}^{-1}$. Bar represents $50 \mu \mathrm{m}$. 
A) $0.5 \%$ GMS

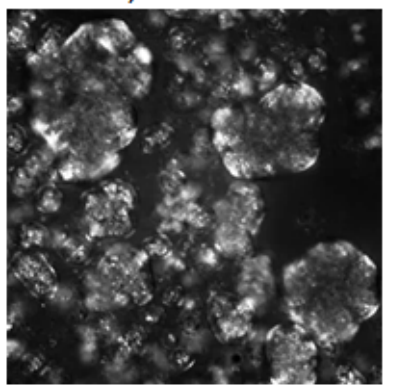

E) $0.5 \%$ GMP

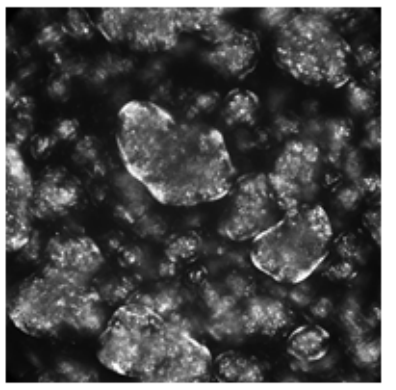

I) $0.5 \% \mathrm{SMS}$

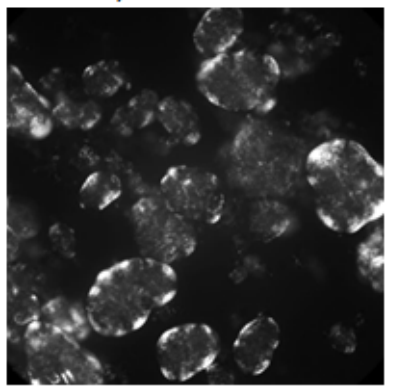

M) $0.5 \%$ STS

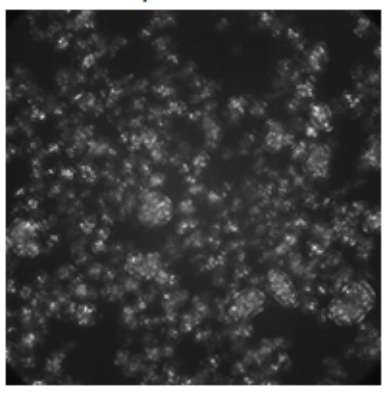

B) $1.5 \%$ GMS

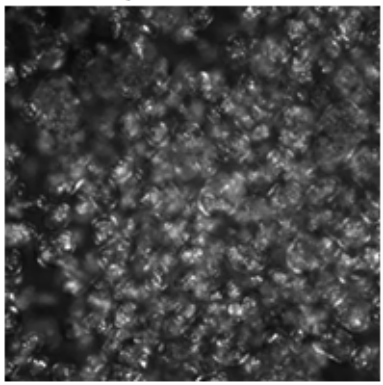

F) $1.5 \% \mathrm{GMP}$

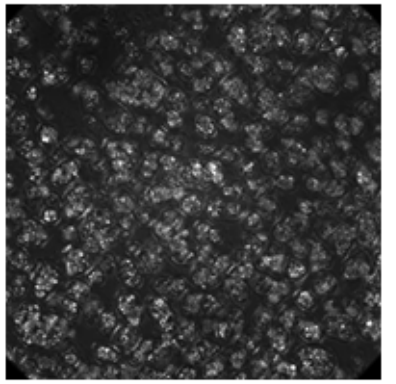

J) $1.5 \% \mathrm{SMS}$

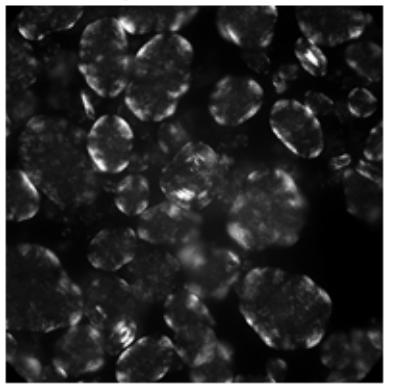

N) $1.5 \%$ STS

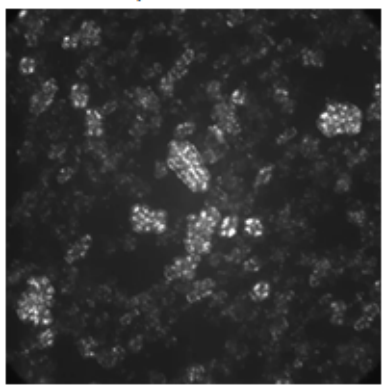

C) $2.5 \% \mathrm{GMS}$

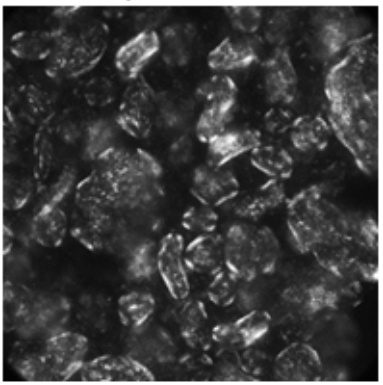

G) $2.5 \% \mathrm{GMP}$

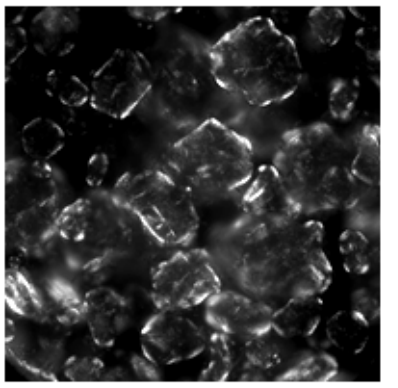

K) $2.5 \% \mathrm{SMS}$

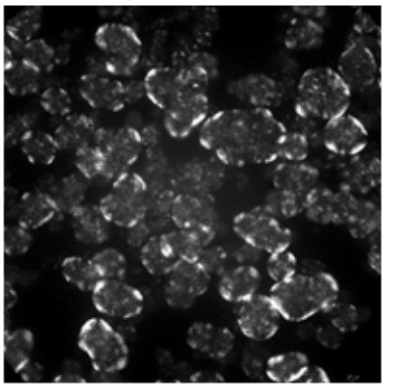

O) $2.5 \%$ STS

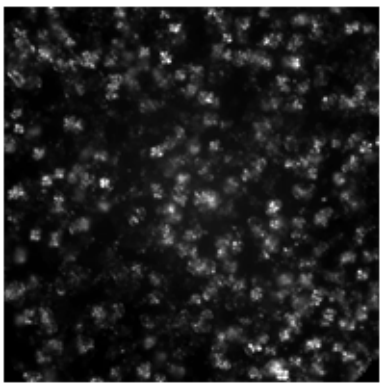

D) $5.0 \%$ GMS

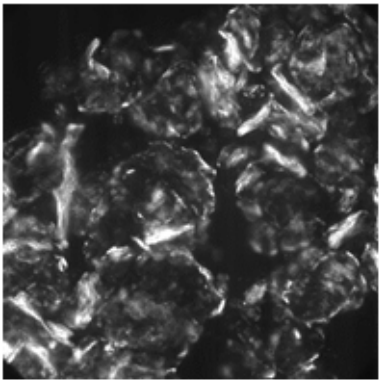

H) $5.0 \% \mathrm{GMP}$

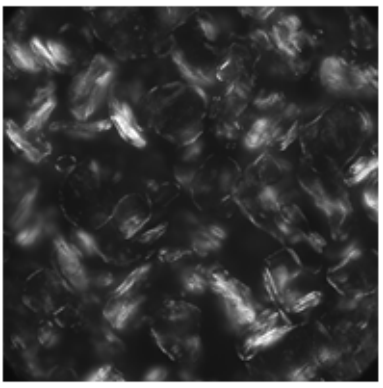

L) $5.0 \% \mathrm{SMS}$

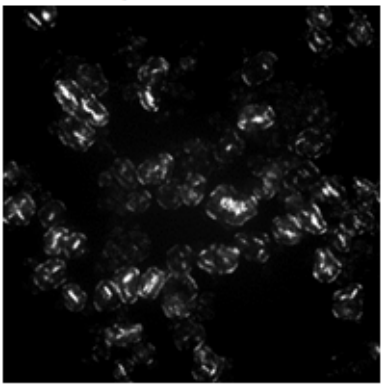

P) $5.0 \% \mathrm{STS}$

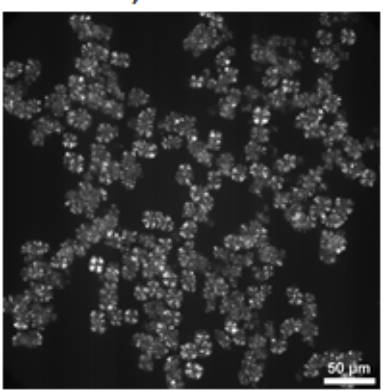

Figure 3. Crystal microstructure of emulsifier-FHCO-CO blends cooled at $1.0^{\circ} \mathrm{C} / \mathrm{min}$ and sheared at $1000 \mathrm{~s}^{-1}$. Bar represents $50 \mu \mathrm{m}$. 
The sorbitan-based emulsifiers (SMS and STS) significantly curtailed crystal formation, with their effects scaling as a function of emulsifier concentration. At $0.5 \mathrm{wt} \%$ SMS (Fig. 3I), the crystal spheroids formed were similar to the control whereas at 1.5 and $2.5 \mathrm{wt} \%$, smaller, clustered spheroids formed (Figs. 3J and K). The microstructure at $5.0 \mathrm{wt} \%$ SMS was completely different, with most crystal spheroids having needle-like crystal cores (and some with globular crystalline cores) surrounded by semi-transparent crystal shells (Fig. 3L). Such behaviour resulted from the increased SMS:FHCO ratio causing the SMS to crystallize first, suggesting nucleation and epitaxial growth at the SMS crystal surface through interaction of FHCO fatty acid chains with the SMS stearic acid residues and/or sorbitan-hydroxyl groups.

In contrast to the other emulsifiers, STS significantly affected FHCO fat crystal spheroid morphology at all concentrations. Even at $0.5 \mathrm{wt} \%$, small, globular assemblies with minimal outer crystalline shells were visible (Fig. 3M). Similar crystal morphologies were observed at $1.5 \mathrm{wt} \%$ and $2.5 \mathrm{wt} \%$ STS and there was no further decrease in average crystal size (Fig. $3 \mathrm{~N}$ and O). At 5.0 wt\% STS, the crystals completely lacked a smoothed outer shell and appeared as globular clusters with Maltese cross patterns (Fig. 3P). This was probably due to the molecular similarity between STS and tristearin, which allowed them to co-crystallize into uniform crystals as evidenced by the lack of distinct crystallization events in the corresponding viscosity profile (Fig. 1D) and DSC crystallization thermogram where only a single crystallization peak was observed (data not shown). Although the action of shear promoted the formation of globular crystals, they were unable to form spheroids. These results suggested that not all fat mixtures may readily form welldefined spheroids under the imposition of shear. 


\subsection{Thermal Analysis}

Table 2 shows the crystallization temperatures of the FHCO, the $10 \mathrm{wt} \%$ FHCO-CO blend as well as the emulsifiers. Shear crystallization had a significant effect on the melting behaviour of the FHCO spheroids (Fig. 4). When crystallized at $1.0^{\circ} \mathrm{C} / \mathrm{min}$ statically $\left(0 \mathrm{~s}^{-1}\right)$, melting peaks occurred at $\sim 58^{\circ} \mathrm{C}$ and a slight peak at $\sim 69^{\circ} \mathrm{C}$, indicating the presence of $\beta^{\prime}$ and some $\quad \beta$ polymorph (Table 3). All sheared samples showed two major endothermic peaks at $\sim 49^{\circ} \mathrm{C}$ and $\sim 60{ }^{\circ} \mathrm{C}$ which were attributed to the $\alpha$ and $\beta^{\prime}$ polymorphs, respectively, with the lesser peak at $\sim 71.5^{\circ} \mathrm{C}$ indicating the presence of a minimal amount of $\beta$ crystals (Table 3).

Table 2. Bulk crystallization temperatures $\left({ }^{\circ} \mathrm{C}\right)$ of $\mathrm{FHCO}$, the $10 \mathrm{wt} \%$ FHCO-CO blend and emulsifiers measured via DSC $(n=3)$.

\begin{tabular}{cc}
\hline Component(s) & Crystallization Temperature $\left({ }^{\mathbf{o}} \mathbf{C}\right)$ \\
\hline FHCO $(100 \mathrm{wt} \%)$ & $74.6 \pm 0.1$ \\
FHCO $(10 \mathrm{wt} \%)$ in CO $(90 \mathrm{wt} \%)$ & $59.8 \pm 0.3$ \\
GMS $(100 \mathrm{wt} \%)$ & $75.2 \pm 0.1,36.9 \pm 0.1$ \\
GMP $(100 \mathrm{wt} \%)$ & $78.3 \pm 0.3$ \\
SMS $(100 \mathrm{wt} \%)$ & $60.9 \pm 0.1$ \\
STS $(100 \mathrm{wt} \%)$ & $61.5 \pm 0.4$ \\
\hline
\end{tabular}




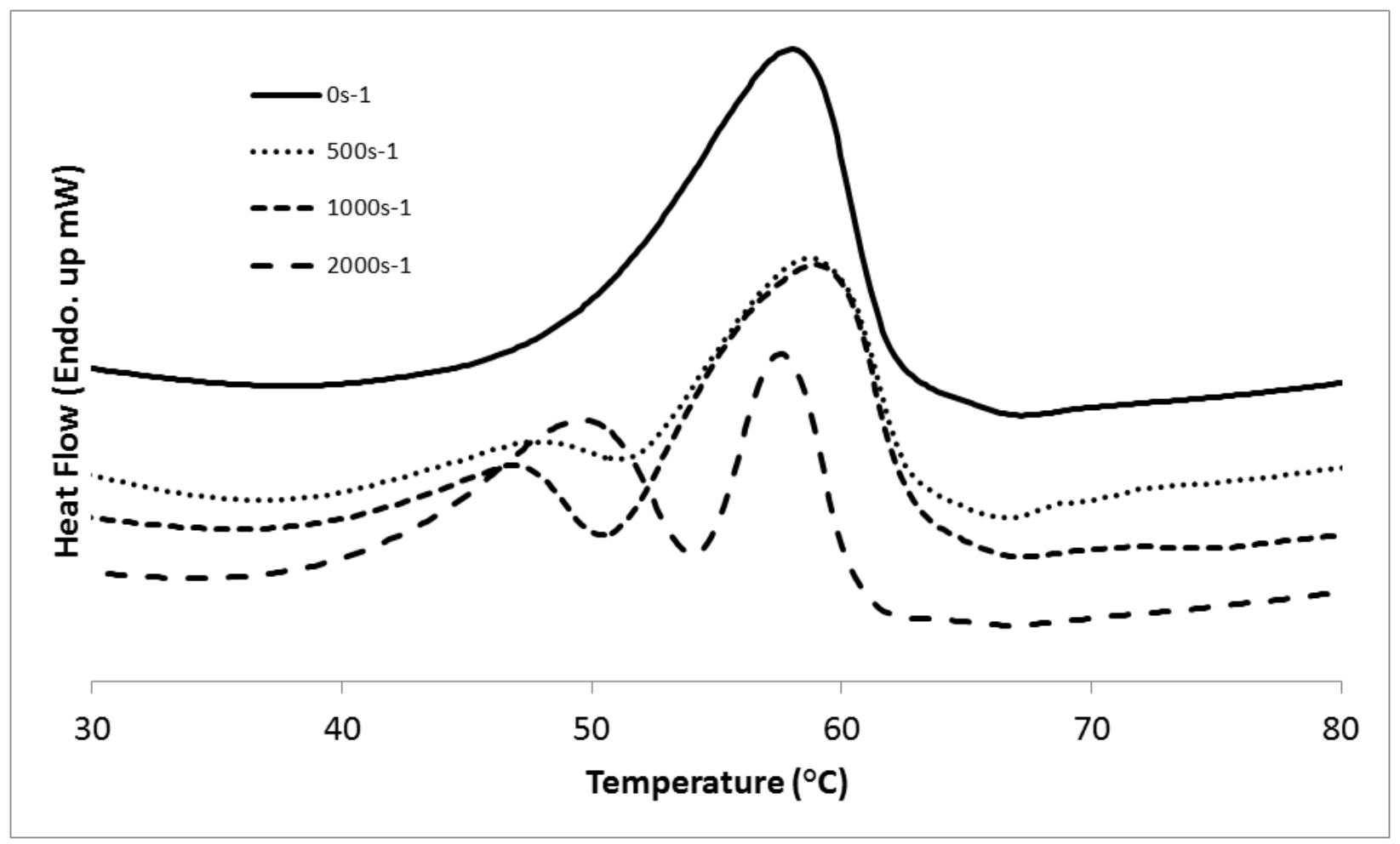

Figure 4. Melting thermograms of $10 \mathrm{wt} \%$ FHCO in $\mathrm{CO}$ blends crystallized at different shear rates.

Table 3. Average melting temperatures of $10 \mathrm{wt} \%$ FHCO in CO blends crystallized at different shear rates $(n=3)$.

\begin{tabular}{ccc}
\hline Shear rate $\left(\mathbf{s}^{-\mathbf{1}}\right)$ & Number of peaks & Peak temperatures $\left({ }^{\mathbf{0}} \mathbf{C}\right)$ \\
\hline 0 & 2 & $57.9 \pm 0.1,69.0 \pm 0.2$ \\
500 & 3 & $48.9 \pm 0.3,61.0 \pm 0.2,69.5 \pm 0.3$ \\
1000 & 3 & $48.2 \pm 1.7,59.8 \pm 1.3,70.9 \pm 0.7$ \\
2000 & 3 & $48.6 \pm 1.1,57.9 \pm 0.4,64.4 \pm 0.1$ \\
\hline
\end{tabular}

Wide-angle x-ray diffraction studies of shear-crystallized control blends showed short spacing peaks at 4.24 and $3.80 \AA$ at room temperature, indicating the $\beta^{\prime}$ polymorph. Literature values list 
the $\alpha, \beta^{\prime}$, and $\beta$-form melting points of pure tristearin at 54,64 , and $73{ }^{\circ} \mathrm{C}$, respectively (Lutton, 1945), which differ significantly from the melting points determined in this work. This was due to the use of FHCO instead of tristearin, which contained other TAGs species (notably $\sim 13 \% \mathrm{C}_{52}$ species with lower melting points) and/or the volume fraction used (10 wt $\%$ FHCO in $90 \mathrm{wt} \% \mathrm{CO}$ vs. $100 \mathrm{wt} \%$ tristearin), where solubilization of high-melting TAGs in CO occurred (Zhou \& Hartel, 2006). Still, shear caused the formation of at least two different melting fractions compared to the single peak in the bulk blend, clearly demonstrating that shear induced the formation of lower-stability polymorphs, most likely as a consequence of increased nucleation resulting in a larger number of smaller $\alpha$ crystals (Hartel, 2001). Shear rate, however, did not affect melting temperatures but did change the ratio of the lower-melting $\alpha$ fraction to the higher-melting $\beta^{\prime}$ fraction with higher shear rates resulting in an increased amount of $\alpha$ polymorph. This suggested that shear may influence the interactions between TAGs at a molecular level, affecting the type of nanoplatelets formed and their interactions to form crystallites and subsequent crystals (Acevedo \& Marangoni, 2014b; MacMillan \& Roberts, 2002; Maleky \& Marangoni, 2008). 

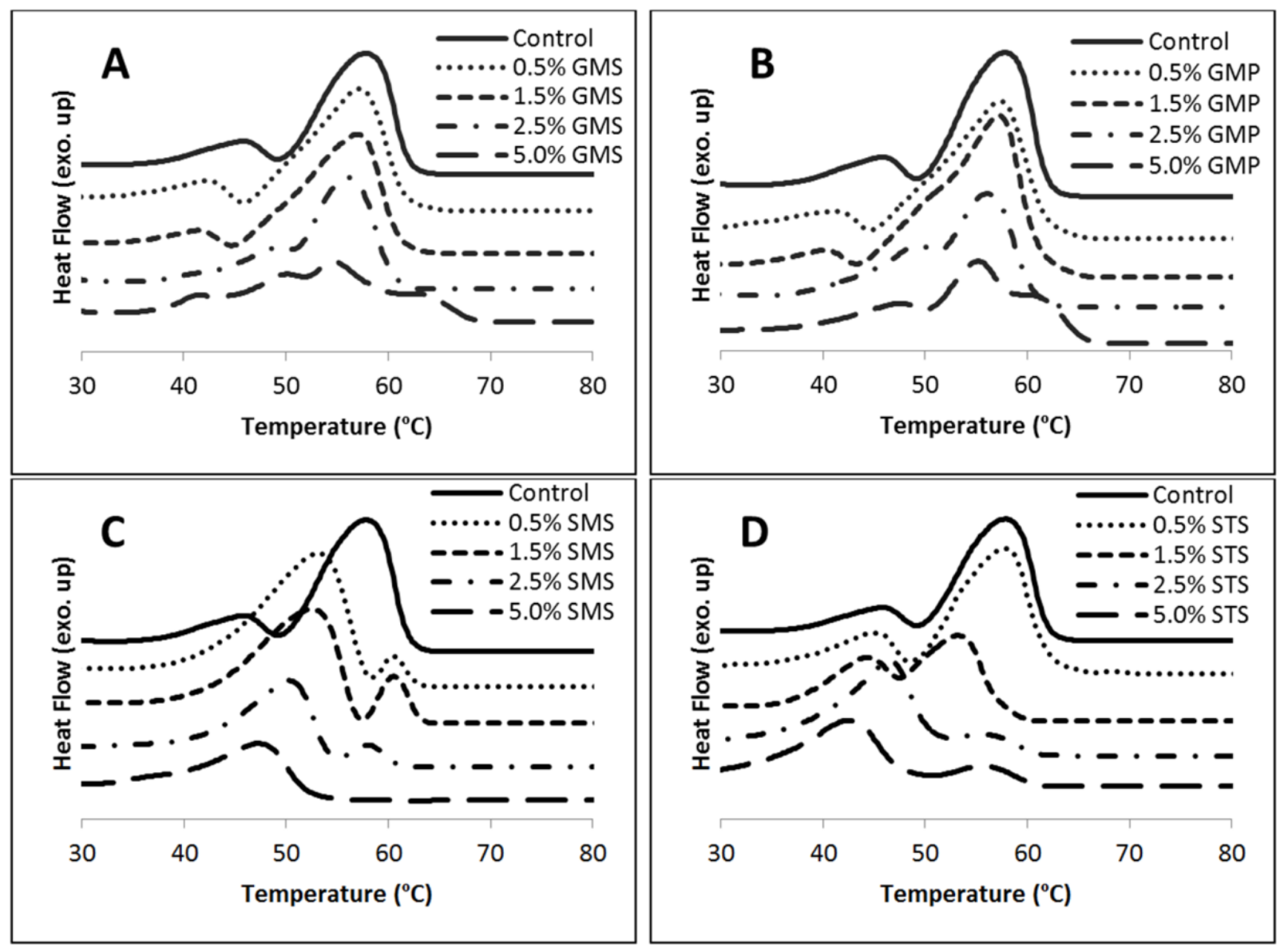

Figure 5. DSC melting profiles of emulsifier-FHCO-CO blends at different concentrations: A) GMS; B) GMP; C) SMS; D) STS. Samples were sheared at $1000 \mathrm{~s}^{-1}$.

Presence and concentration of the emulsifiers greatly affected sheared FHCO melting profiles (Fig. 5). Besides the lower melting temperatures of the two fractions, there were no notable differences in melting behaviour with 0.5 and $1.5 \mathrm{wt} \%$ GMS or GMP. At $2.5 \mathrm{wt} \%$, melting behaviour changed significantly, with the endotherm of both fractions decreasing and the lower-melting fraction becoming less distinct with both MAGs. We attributed this to emulsifier concentration effects where co-crystallization at lower weight fractions evolved towards separate emulsifier and TAG crystallization events resulting from emulsifier saturation of the FHCO crystal lattice, as noted with the viscosity and microscopy results. The lower-melting fraction was attributed to crystallized 
MAG based on DSC melting profiles of $5 \mathrm{wt} \%$ MAG in $95 \% \mathrm{CO}$ blends (data not shown) and the higher melting portion was to co-crystallized MAG and FHCO. At the highest emulsifier concentration (5.0 wt \%), the GMS-containing fat blend exhibited four distinct melting peaks at: i) $\sim 41^{\circ} \mathrm{C}$ (GMS crystals), ii) $\sim 49.5^{\circ} \mathrm{C}$ (mixed GMS-FHCO $\alpha$-crystals), iii) $\sim 55^{\circ} \mathrm{C}$ (GMS-FHCO $\beta^{\prime}-$ mixed crystals) and iv) $\sim 64{ }^{\circ} \mathrm{C}$ (GMS-FHCO $\beta$-mixed crystals). These suppositions were based on microscopy and DSC data, which showed at least two distinct types of crystals at $5.0 \mathrm{wt} \%$ GMS (Fig. 3D). The behaviour of $5.0 \mathrm{wt} \%$ GMP differed from GMS, with three melting fractions at $\sim 47.2, \sim 55$, and $\sim 61{ }^{\circ} \mathrm{C}$ attributed to GMP crystals, mixed GMP-FHCO $\alpha$-crystals and mixed GMP-FHCO $\beta^{\prime}$ crystals, respectively.

The difference in the number of melting fractions observed may be due to differences in chain length between GMS and GMP. As GMS has the same chain length as tristearin (the main component of FHCO) it was better-incorporated into the FHCO crystal lattice due to increased van der Waals interactions between alkyl chains. GMP, having a shorter chain length, caused vacancies in the FHCO crystal lattice when incorporated, which allowed for a higher degree of freedom of movement of the methyl end groups in FHCO's fatty acid chains. This favoured orthorhombic packing resulting in the stabilization of lower-melting crystal polymorphs (Aronhime et al., 1988; Garti, Aronhime, \& Sarig, 1989).

With SMS, two melting fractions were present at all concentrations, with the ratio of the lowermelting to higher-melting fractions dramatically shifting towards the former even at $0.5 \mathrm{wt} \%$ emulsifier (Fig. 5C). This trend scaled with increased SMS concentration with the higher-melting fraction disappearing completely at $5.0 \mathrm{wt} \%$. Such behaviour was attributed to the sorbitan groups hydrogen-bonding with neighbouring TAG molecules, thus preventing their molecular 
reorganization and polymorphic transitions, resulting in the stabilization of lower-melting mixed SMS-FHCO crystals (Aronhime, Sarig, \& Garti, 1987; Aronhime et al., 1988).

A shift towards the lower-melting fraction also occurred with increased STS concentration, although there were still two distinct melting fractions even at $5.0 \mathrm{wt} \%$ (Fig. 5D). Polarized light microscopy only revealed one type of crystal morphology, so it was not possible to attribute the different melting fractions to different crystal types. The lower-melting fraction was likely due to mixed STS-FHCO $\alpha$-crystals and the higher one consisted of mixed STS-FHCO $\beta^{\prime}$-crystals.

\subsection{Shear-Crystallization of Emulsifier-CO Blends}

To elucidate the identity of these different melting fractions, mixtures of emulsifier-CO (no FHCO) were subjected to the same shear-cooling treatments as the FHCO-containing blends. Shear-crystallization of $5 \mathrm{wt} \%$ emulsifier $+95 \mathrm{wt} \% \mathrm{CO}$ blends revealed that all of the emulsifiers tested were generally susceptible to shear forces (Figs. 6B, E, H and K). Samples subjected to shear showed smaller, globular, distinct crystals compared to statically-cooled emulsifier-oil blends (Figs. 6C, F, I and L). This was in agreement with work from other groups that showed that shear can reduce crystal size either by breaking larger crystals or by enhancing nucleation while inhibiting cluster growth and aggregation (Acevedo et al., 2012; Kloek et al., 2005; Maleky et al., 2011). Shear-crystallization of the MAGs resulted in roughened globular crystals with GMS-based blends appearing more crystalline than GMP-based on PLM. Un-sheared GMS samples consisted of clustered feather-like crystalline masses whereas GMP crystallized mainly as distinct needlelike crystals with a more uniform size distribution. Such a difference in crystallization behaviour may have affected the crystal aggregation behaviour during shearing, with GMS having a greater propensity to aggregate, thus resulting in denser, more crystalline globular crystal masses compared to GMP. Comparing the emulsifier + oil blends to blends also containing FHCO (Fig. 
6A and D), we attributed the roughened crystals discussed earlier to the MAGs. This also supports the theory that the longer tube-like crystals observed in the blends containing FHCO were MAGFHCO crystal composites since they were absent in blends without FHCO.

The sorbitan-containing emulsifiers (SMS and STS) were also susceptible to shear during crystallization, showing globular microstructures compared to un-sheared samples (Fig. 6H and $\mathrm{K}$ vs. Fig. $6 \mathrm{I}$ and L, respectively) but they did not exhibit the roughened microstructure seen in the MAGs. This suggested that the sorbitan group hindered formation of spheroidal crystals, perhaps as a result of differences in molecular packing, with the smaller glycerol group allowing the MAGs to more easily form lamellae/nanoplatelets. Upon comparison of the microstructures of samples with and without FHCO (Fig. G, H, J, K), there were similarities between the needle-like crystal cores of sheared SMS-FHCO-CO blends vs. the sheared SMS-CO blends as well as the sheared STS-FHCO-CO crystals vs sheared STS-CO blends, strongly supporting our hypothesis that the sorbitan emulsifiers nucleated first and acted as templates for epitaxial FHCO crystallization. 
A) $5 \%$ GMS $5 \% \mathrm{HCO} 90 \% \mathrm{CO}$ $\left(1000 \mathrm{~s}^{-1}\right)$

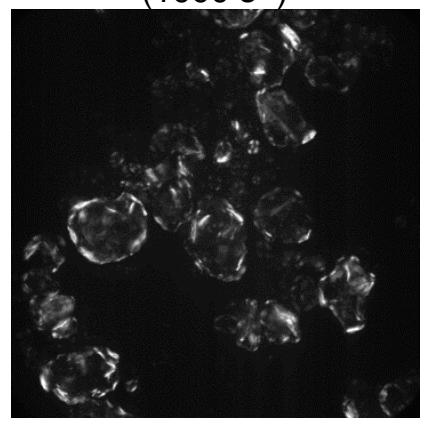

D) $5 \%$ GMP $5 \% \mathrm{HCO} 90 \% \mathrm{CO}$ $\left(1000 \mathrm{~s}^{-1}\right)$

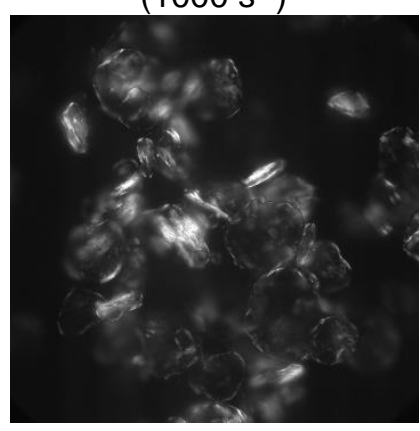

G) $5 \% \mathrm{SMS} 5 \% \mathrm{HCO} 90 \% \mathrm{CO}$

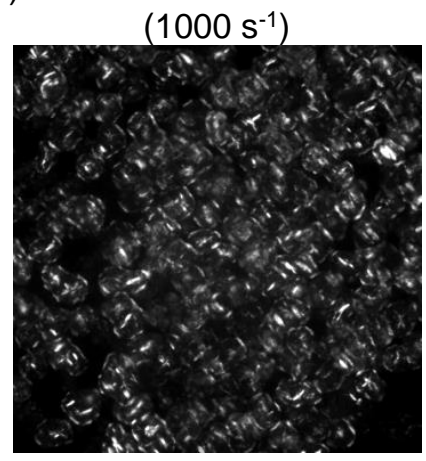

J) $5 \%$ STS $5 \% \mathrm{HCO} 90 \% \mathrm{CO}$ $\left(1000 \mathrm{~s}^{-1}\right)$

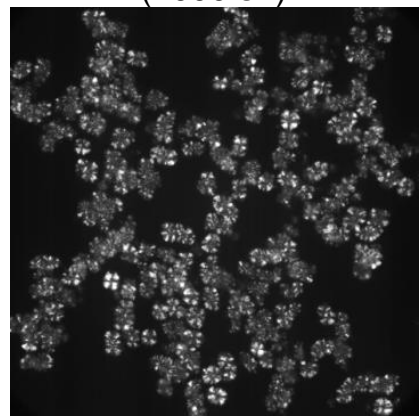

B) $5 \%$ GMS $95 \% \mathrm{CO}$

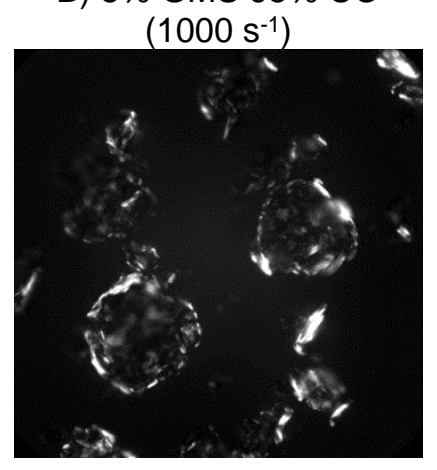

E) $5 \%$ GMP $95 \% \mathrm{CO}$ $\left(1000 \mathrm{~s}^{-1}\right)$

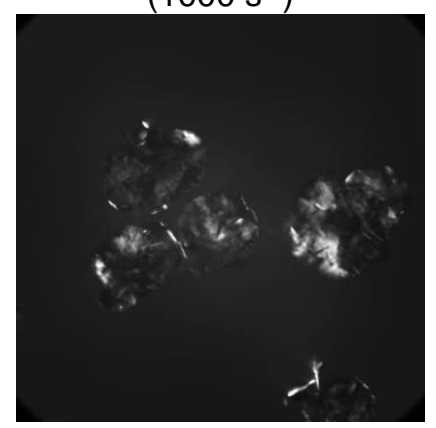

H) $5 \% \mathrm{SMS} 95 \% \mathrm{CO}$ $\left(1000 \mathrm{~s}^{-1}\right)$

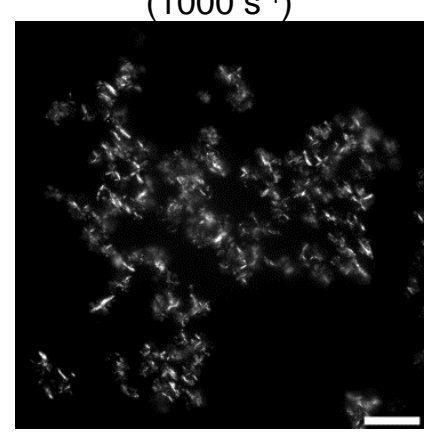

K) $5 \%$ STS $95 \%$ CO $\left(1000 \mathrm{~s}^{-1}\right)$

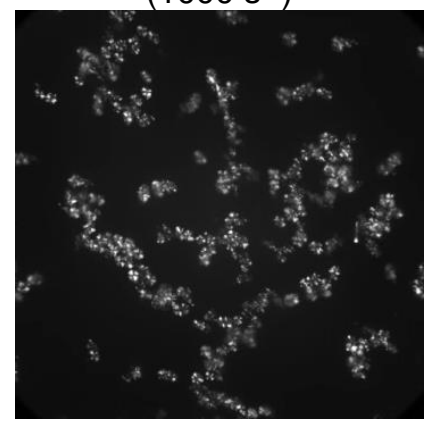

C) $5 \%$ GMS $95 \%$ CO

$\left(0 \mathrm{~s}^{-1}\right)$

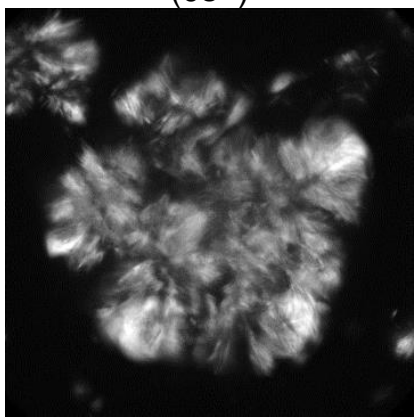

F) $5 \%$ GMP $95 \% \mathrm{CO}$ $\left(0 \mathrm{~s}^{-1}\right)$

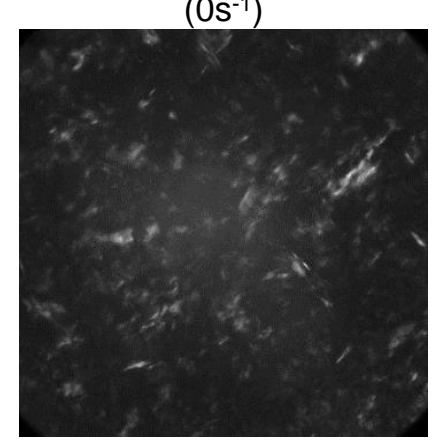

I) $5 \% \mathrm{SMS} 95 \% \mathrm{CO}$ $\left(0 s^{-1}\right)$

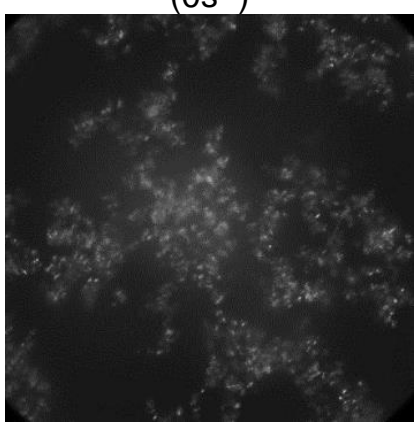

L) $5 \%$ STS $95 \%$ CO $\left(0 \mathrm{~s}^{-1}\right)$

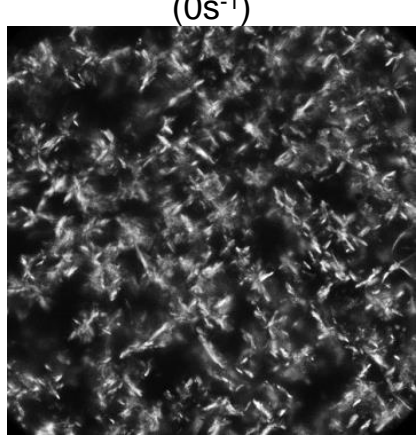

Figure 6. Shear-crystallized blends of emulsifier + oil blends. Bar represents $50 \mu \mathrm{m}$. 


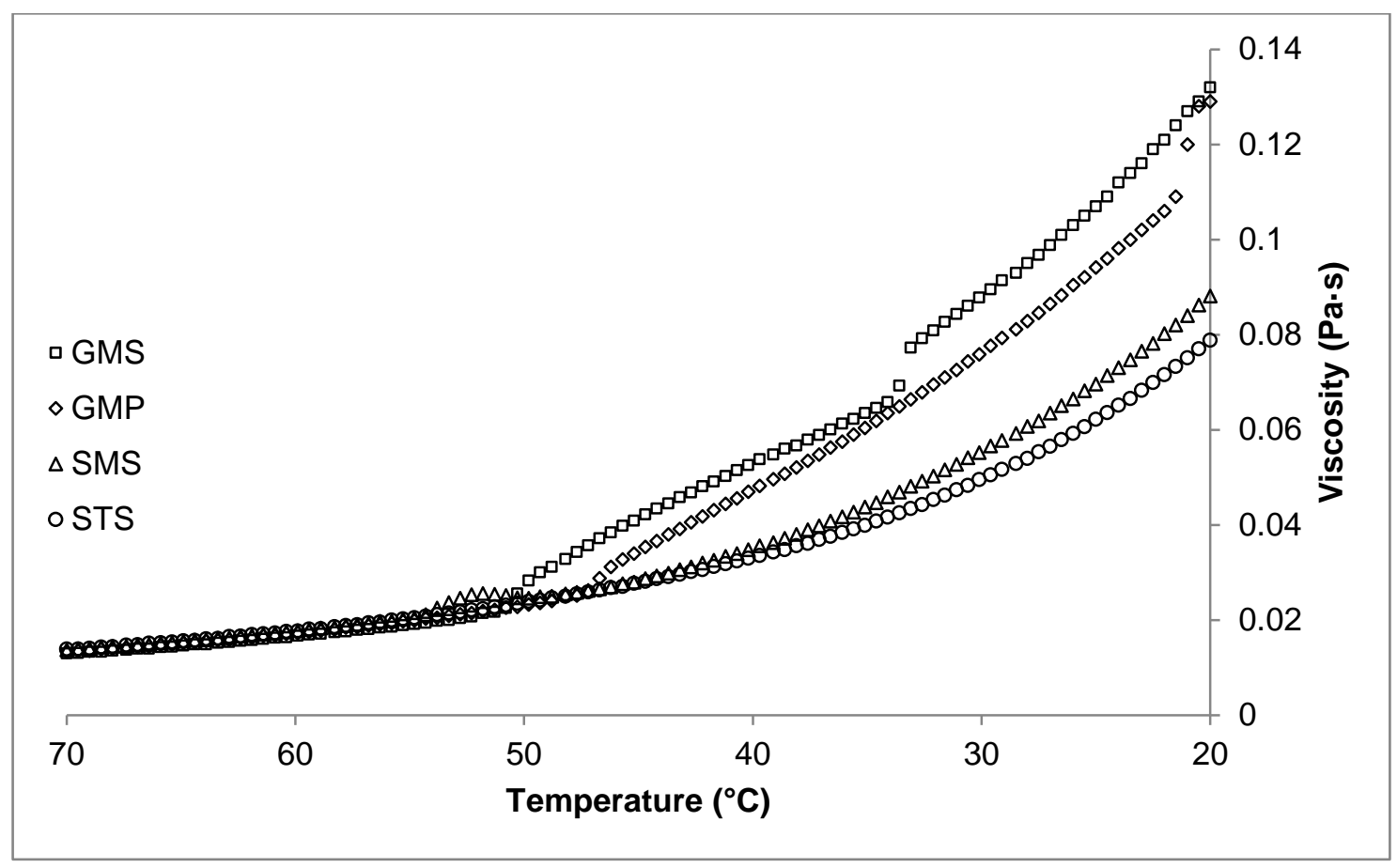

Figure 7. Viscosity profiles of $5 \mathrm{wt} \%$ emulsifier $95 \mathrm{wt} \% \mathrm{CO}$ blends cooled at $1.0{ }^{\circ} \mathrm{C} / \mathrm{min}$ while sheared at $1000 \mathrm{~s}^{-1}$.

The viscosity profiles of sheared emulsifier-CO blends generally showed different trends compared to the emulsifier-FHCO-CO blends (Fig. 1 vs. Fig. 7). Based on viscosity increases, there were no significant differences in initial crystallization temperatures compared to the FHCOcontaining blends (Table 1) $(\mathrm{P}>0.05)$, but there were differences in second-stage crystallization onset $(\mathrm{P}<0.05)$. The GMS-FHCO-CO blends showed a slight viscosity increase at $\sim 38{ }^{\circ} \mathrm{C}$ and a more pronounced increase at $\sim 29^{\circ} \mathrm{C}$ while GMS-CO blends (no FHCO) showed a second viscosity increase at $\sim 34^{\circ} \mathrm{C}$ (Fig. 7 square). Similarly, GMP-FHCO-CO blends showed a second-stage viscosity increase at $\sim 38^{\circ} \mathrm{C}$ while GMP-CO blends showed a secondary increase at $\sim 22^{\circ} \mathrm{C}$ (Fig. 7 circle). SMS-FHCO-CO blends showed a secondary increase in viscosity at $\sim 30^{\circ} \mathrm{C}$ which was absent in SMS-CO blends. STS blends, both with and without FHCO, showed similar 
crystallization trends where there was a gradual increase in viscosity with time/temperature and with no observable jumps in viscosity, demonstrating that the STS greatly hindered FHCO crystallization and did not extensively crystallize alone. Control blends without emulsifier showed primary crystallization at $\sim 37^{\circ} \mathrm{C}$ (Fig. 1). The higher primary crystallization temperatures for blends containing emulsifiers support our hypothesis that the emulsifiers crystallized before FHCO and acted as seeds for further epitaxial crystal growth.

\subsection{Mechanism}

In previous work, we proposed a mechanism for the formation of crystal spheroids (Tran et al., 2014). To recap, crystal spheroid formation was defined as a multi-step process consisting of: i) potential shear-induced pre-structuring of TAGs in the melt; ii) nucleation and formation of nanoplatelets (with relatively large distances between nanoplatelets and thus a low degree of interparticle interactions); iii) rotation and tumbling of platelets due to shear in the flow field; iv) nanoplatelet aggregation into extended stack-like structures; v) clustering of the nanoplatelet stacks into globular aggregates or bundles, depending on the shear rate; vi) crystallization into clusters of $\alpha$-crystals while tumbling, forming crystal spheroids; vii) shear acceleration of the $\alpha \rightarrow \beta^{\prime}$ (or $\beta$ ) polymorphic transition and finally viii) viscous heat generation causing melting of looselyassociated fat crystals at the spheroid surface, resulting in smoother surfaces.

Emulsifiers may promote fat crystallization via templating effects or hinder it by blocking growing sites on crystal surfaces during nucleation and/or crystal growth (Shimamura et al., 2013; Smith et al., 2011; Verstringe, Dewettinck, et al., 2014). The impact of emulsifiers on crystallization may be affected by concentration, cooling rate, and/or the presence of agitation (shear) (Gordon \& Rahman, 1991; D. Rousseau et al., 2005). For the four systems in this study, the viscosity profiles (Fig. 1) showed reductions in crystallization onset temperature thus no independent emulsifier 
crystallization occurring for low concentrations $(\leq 1.5 \mathrm{wt} \%$ for MAGs, $2.5 \mathrm{wt} \%$ for sorbitanbased).

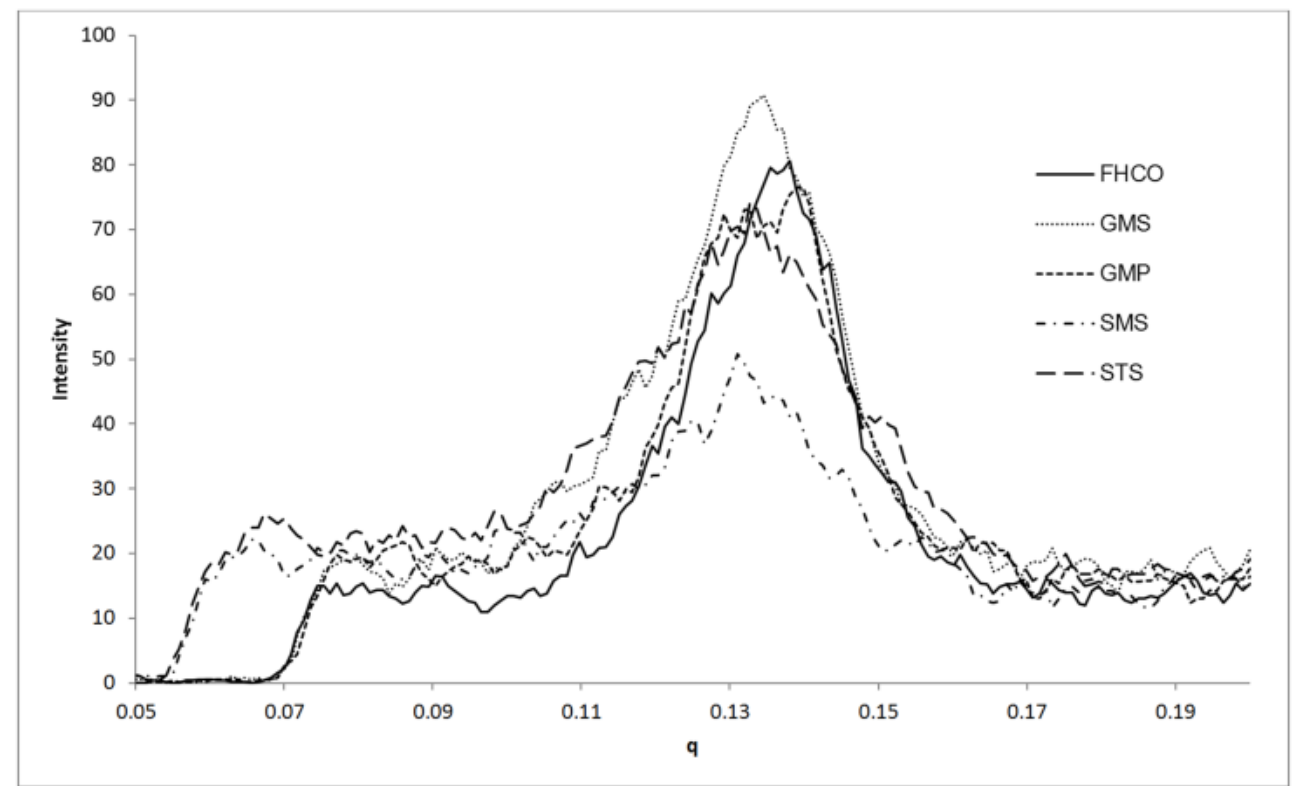

Figure 8. SAXD spectra of the control (10 wt $\%$ FHCO) and $1.5 \mathrm{wt} \%$ emulsifier $+8.5 \mathrm{wt} \% \mathrm{FHCO}$ blends in $\mathrm{CO}$ at $20^{\circ} \mathrm{C}$.

SAXD results (Fig. 8) corroborate this observation; the peak position obtained from the control system is unchanged by the addition of $1.5 \mathrm{wt} \%$ emulsifier. Conversely, for the samples containing $5.0 \mathrm{wt} \%$ emulsifier, the SAXD peak was clearly influenced, where peak locations shifted in the direction of the emulsifier-CO blend peak (Fig. 9). Shifts were more pronounced $(+5 \AA$ ) for the sorbitan-based emulsifiers; corresponding WAXD indicated the resulting crystals were likely the $\alpha$-polymorph (data not shown), further demonstrating the ability of the sorbitan group to stabilize lower polymorphs. The MAGs, on the other hand, showed slightly smaller peak shifts (+ $3.5 \AA$ ), which we ascribed to the smaller glycerol group. Further, we used GMS and GMP to study the effect of chain length (18C vs 16C) on the formation of crystal spheroids. We found that GMS, 
having the same acyl chain as tristearin (the main component of FHCO), had a greater effect on crystal spheroid microstructure than GMP. This was in agreement with reports that the effect of an emulsifier is greatest when its chain length matches that of the fat; with the effect decreasing rapidly for shorter chain lengths (Smith et al., 2011). WAXD further confirmed this, where GMS showed peak spacings for $\beta$ ' whereas GMP did not (data not shown). It has also been reported that sorbitan esters such as SMS and STS optimally co-crystallize with a fat when their acyl chains match (Garbolino, Bartoccini, \& Floter, 2005). While SMS and STS had significant effects on crystal microstructure, no further differences between the two could be ascertained from XRD.
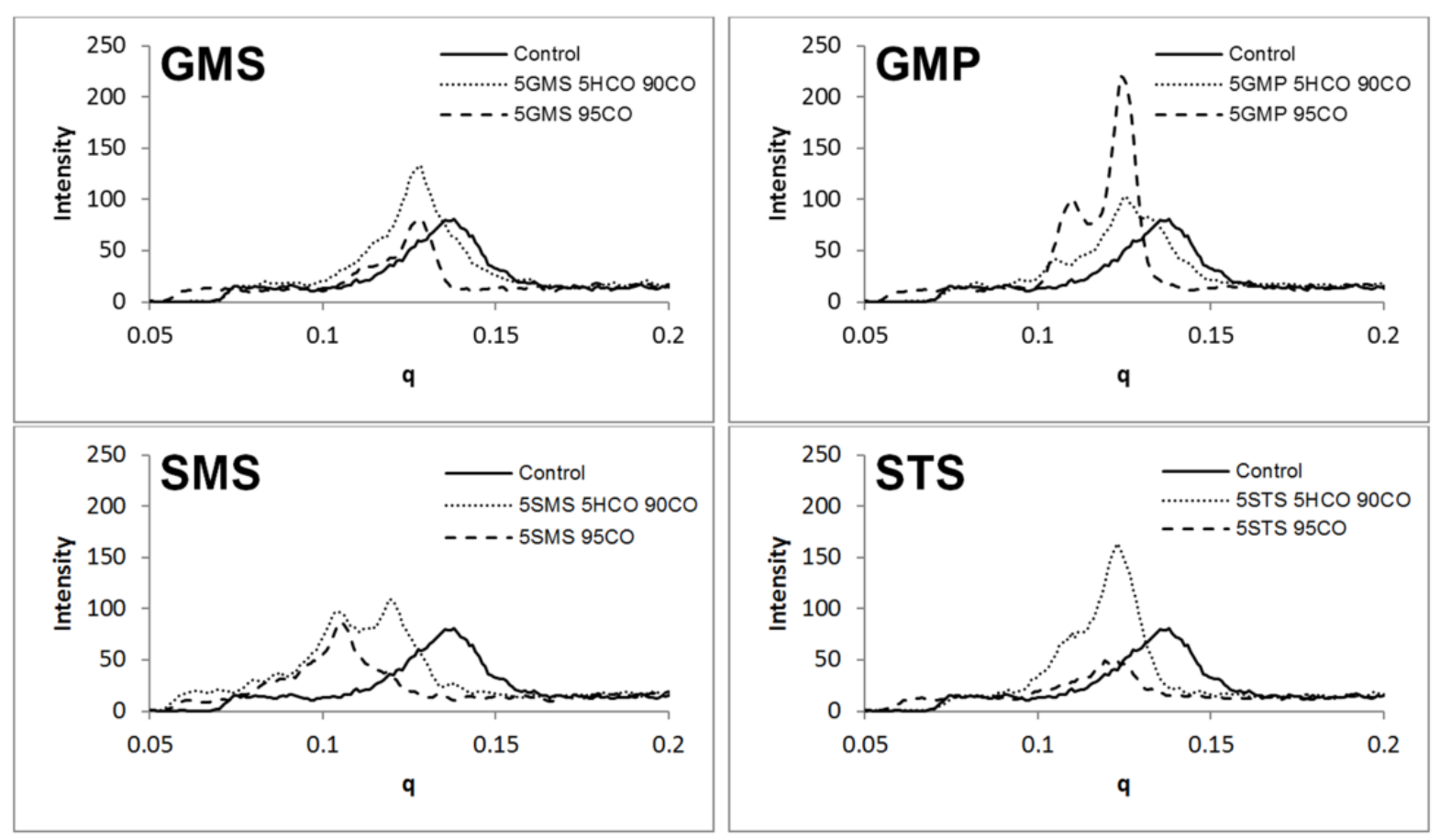

Figure 9. SAXD spectra of the control (10 wt $\%$ FHCO), blends of $5 \mathrm{wt} \%$ emulsifier $+5 \mathrm{wt} \%$ $\mathrm{FHCO}+90 \mathrm{wt} \% \mathrm{CO}$, and blends of $5 \mathrm{wt} \%$ emulsifier $+95 \mathrm{wt} \% \mathrm{CO}$ taken at $20{ }^{\circ} \mathrm{C}$.

Shear has been shown to alter TAG nucleation at a molecular level, causing crystal nanoplatelets to elongate and orient in the flow direction (Acevedo \& Marangoni, 2014b; MacMillan \& Roberts, 
2002; Maleky \& Marangoni, 2008). This has also been supported by computer simulations (Marangoni et al., 2012; Peyronel et al., 2013; Pink et al., 2013). Below a critical value, shear may enhance crystal nucleation and growth and accelerate polymorphic transitions toward more stable phases while above this critical value, shear may hinder crystal growth by impeding fat crystal aggregation and/or breaking them down (Kloek et al., 2005; MacMillan \& Roberts, 2002; Mazzanti et al., 2003, 2011, 2005; Mazzanti, Marangoni, \& Idziak, 2008; Sonwai \& Mackley, 2006).

In an effort to further elucidate the effects of shear in conjunction with emulsifiers, we showed the impact of shear $\left(500,1000\right.$ or $\left.2000 \mathrm{~s}^{-1}\right)$ on different blends $(0.5,1.5$, and $2.5 \mathrm{wt} \%$ emulsifier mixed with $90 \mathrm{wt} \% \mathrm{CO}$, the remainder wt $\%$ being $\mathrm{FHCO}$ ). For all emulsifiers, shear rate did not affect primary crystallization temperatures but did show decreased viscosities with higher shear rates (data not shown). These findings were similar to previously-reported results for FHCO-CO blends without emulsifier (Tran et al., 2014). 

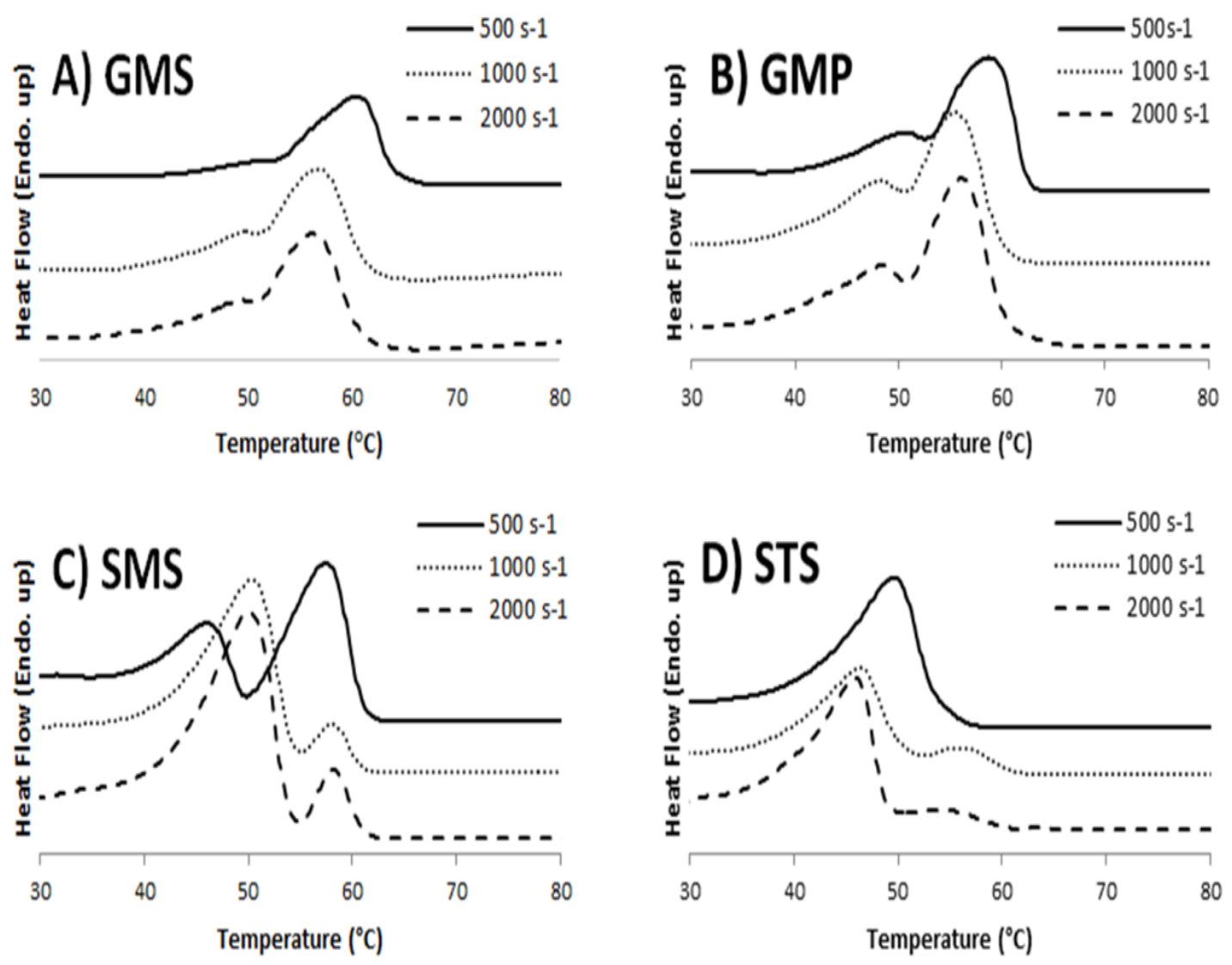

Figure 10. DSC melting thermograms showing the effect of shear rate on blends of wt $\% \mathrm{CO}, 7.5 \mathrm{wt} \% \mathrm{FHCO}$, and $2.5 \mathrm{wt} \%$ emulsifier cooled at $1.0^{\circ} \mathrm{C} / \mathrm{min}$.

DSC analyses revealed that higher shear forces caused a change in the relative ratios of the higher and lower-melting ones fractions (Fig. 10) with these effects being emulsifier-dependent. The two MAGs showed similar susceptibilities to shear: the ratio of the lower-melting to higher-melting fractions increased with shear rate up to $1000 \mathrm{~s}^{-1}$ for both GMS (Fig. 10A) and GMP (Fig. 10B), suggesting a critical shear rate above which higher shear forces do not have significant effects on crystal melting behaviour. The sorbitan-based emulsifiers (SMS and STS) showed similar trends with shifts to lower-melting fractions with higher shear rates (Figs. 10C and D, respectively). Shear rate significantly enhanced the incorporation of SMS at $1000 \mathrm{~s}^{-1}$ and above, while these effects 
were less pronounced for STS. Instead, STS exhibited more pronounced higher-melting fractions with increased shear rate, which could be due to possible saturation of the crystal matrix leading to the separate crystallization of FHCO and/or STS. Alternatively, it may be due to the opposite, where higher shear forces may hinder diffusion of the emulsifier to the crystal surface thus resulting in a shift from favouring crystal aggregation and growth to nucleation instead, where it is possible that partitioning of the FHCO and STS in the liquid state resulted in independent nucleation and crystallization instead of co-crystallization. Overall, the mechanism of crystal spheroid formation was not significantly affected by the incorporation of emulsifiers and followed the regime outlined above, although it was shown that emulsifiers that crystallize before the fat (FHCO) may have templating effects and affect the final morphology of the spheroids.

\section{Conclusion}

We have reported on the formation of laminar shear-induced spheroidal fat crystal assemblies and the impact of four common food emulsifiers on structure development. Notable results indicate that both cooling and shearing are required for the formation of crystal spheroids with the latter having a greater impact. Emulsifier incorporation within the TAG crystal lattice is a highly sheardependent phenomenon, with the role of emulsifier structure, concentration and crystallization temperature key parameters dictating incorporation as a function of laminar shear. Further work is continuing to elucidate the effects of both shear and emulsifiers on the formation of spheroidal fat crystal structures and their applications. 


\section{Chapter 4 - Encapsulation of Water-in-Oil Emulsion Droplets within Crystal Spheroids}

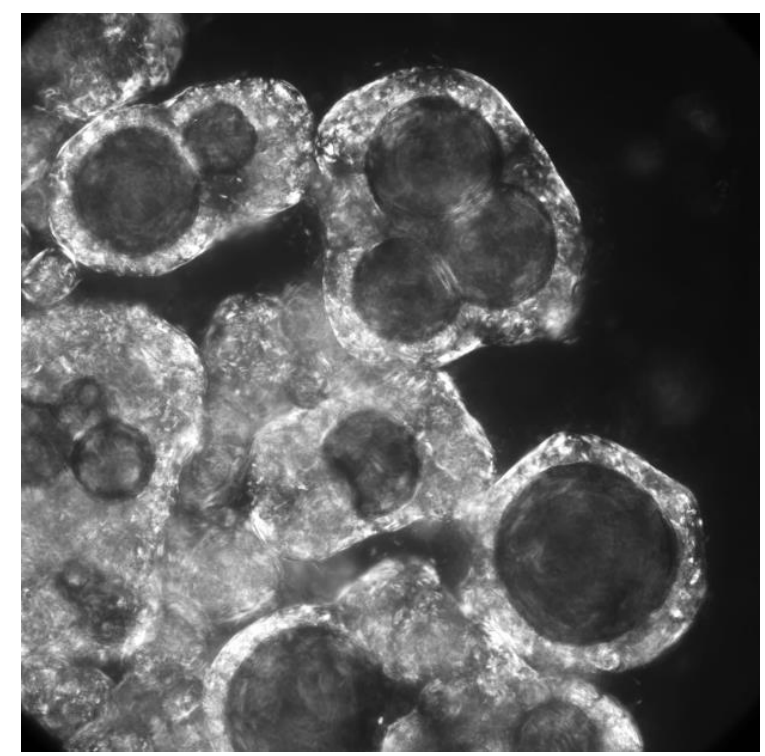

- Shear-crystallization of water-in-oil led to emulsion droplets encapsulated within crystal spheroid Pickering shells

- Crystal spheroid shell formation was dependent on emulsifier molecular similarity and compatibility to the crystallizing fat

- Introduction of a dispersed aqueous phase did not affect crystal polymorph 


\begin{abstract}
Model 10\% (w/w) water-in-oil emulsions were shear-crystallized to produce crystal spheroidencapsulated droplets. The bulk oil phase was composed of canola oil (CO), fully-hydrogenated canola oil (FHCO), and different emulsifiers: glycerol monostearate (GMS), glycerol monopalmitate (GMP), glycerol monooleate (GMO), sorbitan monostearate (SMS), sorbitan tristearate (STS), or polyglycerol polyricinoleate (PGPR). Emulsions were prepared using a rotorstator and then further shear-crystallized using a rheometer with a parallel plate geometry. The efficacy of water droplet encapsulation was dependent on emulsifier type, with the glycerol-based emulsifiers (GMS, GMP, and GMO), due to their molecular similarity with the crystallizing phase (FHCO), producing smooth-surfaced crystal spheroid shells while the sorbitan-based emulsifiers produced irregularly-shaped crystal shells and droplet cores (SMS) or incomplete crystal shell formation (STS). PGPR-based emulsions did not exhibit droplet-encapsulation due to its large molecular difference to the crystallizing phase. Analysis of viscosity profiles, thermal behaviour, and x-ray diffraction revealed that the addition of $\quad 10 \%(\mathrm{w} / \mathrm{w})$ water did not significantly affect crystal polymorphism.
\end{abstract}




\section{Introduction}

Emulsions are important in many industries such as pharmaceuticals (drug delivery systems), cosmetics (beauty products like lipstick, and foods (butter, margarine, and tablespreads) (Borwankar, Frye, Blaurock, \& Sasevich, 1992; Le Révérend, Taylor, \& Norton, 2011; McClements, 2010). Emulsions can be of the oil-in-water (O/W) type, with oil dispersed in a continuous aqueous phase, or vice versa as water-in-oil (W/O) emulsions. The stability and properties of the emulsions depend on their compositions which typically includes water, liquid oil, and emulsifiers. In W/O emulsions, stability may be enhanced via the addition of a crystallizing fat, usually saturated triacylglycerols (TAGs), that may act by network stabilization, where the fat crystals form a sintered 3D network that prevents droplet migration, or by Pickering stabilization, where the fat crystals form solid shells around droplets to prevent coalescence (Ghosh \& Rousseau, 2011; Ghosh et al., 2011; Dérick Rousseau \& Hodge, 2005). Processing conditions, such as heating/cooling rates and agitation (shear) play a major role in determining the physical properties of the fats, such as melting behaviour and polymorphism. The three common polymorphs of TAGS, in order of increasing organization, crystal size, density, and stability are $\alpha, \beta$ ', and $\beta$ (Sato, 2001; Walstra, 2003). Polymorphic characterization can be carried out using a number of techniques, with differential scanning calorimetry (DSC) and x-ray diffraction (XRD) being most common (Himawan, MacNaughtan, Farhat, \& Stapley, 2007; Lavigne, Bourgaux, \& Ollivon, 1993). DSC provides information about the thermal behaviour (melting and/or crystallization) of a sample while XRD characterizes the spatial arrangements of TAG molecules within the crystals which allows for crystal polymorph identification based on characteristic melting or crystallization peak temperatures and spacings between TAG molecules, respectively. Shear forces may have significant effects on fat crystallization. Shear may enhance nucleation, the initial formation of fat 
crystal nanoplatelets, by providing enough energy to overcome activation energy barriers (Hartel, 2001), which typically results in increased crystallization rates and leads to mechanically-stronger crystal networks (Marangoni \& Narine, 2002; Suresh S. Narine \& Marangoni, 1999). Shear, below a critical rate, may promote aggregation of crystallites and enhance crystallization rates resulting in larger crystals whereas above the critical rate, average crystal size may be reduced due to shear breaking down larger crystals, which introduces surfaces for enhanced secondary nucleation, and/or inhibiting growth and aggregation of crystal clusters (Kloek et al., 2005; Maleky \& Marangoni, 2008; Maleky et al., 2011; Tarabukina et al., 2009).

Recently, a new type of Pickering stabilization was characterized by Ghosh et al., where laminar shear was applied to the crystallization of W/O emulsions resulting in water droplets encapsulated within crystal spheroid shells (Ghosh \& Rousseau, 2012; Dérick Rousseau, 2013). Encapsulation efficacy was dependent on emulsifier similarity and compatibility with the crystallizing fat, where glycerol monooleate (GMO), having a higher degree of similarity to tristearin, the main component of their model fat [fully-hydrogenated canola oil (FHCO)], promoted interfacial crystallization of FHCO while polyricinoleate (PGPR), having a much higher molecular weight and highlybranched structure dissimilar to FHCO, did not promote interfacial crystallization. In a recent study, Tran et al. characterized the formation of the crystal spheroids, studied the processing conditions for optimal spheroid crystallization, and proposed a mechanism for their formation (Tran et al., 2014). In a following study, Tran et al. examined the role of the monoacylglycerides (MAGs) glycerol monostearate (GMS) and glycerol monopalmitate (GMP) and the sorbitan-based emulsifiers sorbitan monostearate (SMS) and sorbitan tristearate (STS) on the shear-crystallization of FHCO and found that spheroid formation was significantly impacted by emulsifier type and concentration where GMS, GMP, and SMS significantly reduced spheroid sizes even at low 
concentration $(0.5 \mathrm{wt} \%)$ but caused elongation and deformation of the crystals at higher concentrations (up to $5.0 \mathrm{wt} \%$ ). At $1.5 \mathrm{wt} \%$ and below, the MAGs co-crystallized with FHCO but crystallized separately at higher concentrations (2.5 and 5.0 wt\%) (Tran, Green, \& Rousseau, 2015). In this study, we further investigate the effect of different emulsifier types (monoacylglycerides, sorbitan esters, and PGPR) on the encapsulation of water droplets within crystal spheroid shells and demonstrate that crystal morphology may be tailored via the choice of emulsifier. 


\section{Materials and Methods}

\subsection{Materials}

Canola oil (CO) (acid value $\sim 0.2 \%$ ) was purchased from a local grocery store and stored at room temperature until use. Fully-hydrogenated canola oil (FHCO) was purchased from Bunge (Oakville, ON, Canada) and had a capillary melting point of $69.5^{\circ} \mathrm{C}$ (AOCS official method Cc 1-25) and a free fatty acid content of $0.018 \%$ (AOCS Society, 1998). The predominant TAG species in $\mathrm{FHCO}$ were $\mathrm{C}_{52}(13.3 \%), \mathrm{C}_{54}(73.6 \%)$, and $\mathrm{C}_{56}$ (5.0\%). Distilled GMS (Alphadim 90 SBK) was kindly donated by Corbion Caravan (Mississauga, ON, Canada). Distilled GMP (DIMODAN® HP K-A), GMO (DIMODAN MO 90), and PGPR were obtained from DANISCO USA INC. (Madison, WI, USA). Distilled SMS (Span 60) and STS (Span 65) were purchased from Sigma Aldrich (Saint Louis, MO, USA). All components were used as received from suppliers without further purification.

\subsection{Emulsion Preparation and Shear-Crystallization}

The oil phase of the emulsions was prepared by mixing CO (90 wt $\%$ ), FHCO (8.5 wt $\%$ ), and emulsifier $(1.5 \mathrm{wt} \%)$. The blends were heated to $70{ }^{\circ} \mathrm{C}$ with stirring to melt the FHCO and emulsifiers. Water was heated to $70{ }^{\circ} \mathrm{C}$ and then added to the oil phase resulting in a $10 \mathrm{wt} \%$ aqueous phase and $90 \mathrm{wt} \%$ oil phase mixtures. The mixtures, while hot, were sheared with a rotorstator mixer (PT 10/35, Kinematica Inc., Bohemia, NY, USA) at 27,000 RPM for 1 minute to produce the emulsions. Aliquots were then withdrawn and deposited between the parallel plates (PP25, $25 \mathrm{~mm}$ diameter, $0.5 \mathrm{~mm}$ gap) of a rheometer (Physica MCR301, Anton Paar, Ville StLaurent, QC, Canada). Samples were kept at $70{ }^{\circ} \mathrm{C}$ for $20 \mathrm{~min}$ and then cooled at $1.0^{\circ} \mathrm{C} / \mathrm{min}$ while being simultaneously sheared at $1000 \mathrm{~s}^{-1}$. Apparent viscosity was measured as the samples were sheared and cooled. All experiments were performed at least in triplicate. 


\subsection{Microscopy}

Microstructure was observed via polarized light microscopy (PLM). Immediately upon completion of shear-crystallization, samples were placed on viewing slides at room temperature $\left(\sim 25^{\circ} \mathrm{C}\right)$ (Fisher Scientific, Ottawa, ON, Canada), covered with a cover slip (Fisher Scientific, Nepean, ON, Canada) and analyzed with an inverted light microscope (Axiovert 200M, Zeiss Inc., Toronto, ON, Canada) equipped with a CCD camera (QImaging, Inc., RETIGA 4000R, Surrey, BC, Canada) using QImaging Q-Capture Pro 7 software (Version 7.0.5 Build 4325, QImaging, Inc., Surrey, BC, Canada).

\subsection{Differential Scanning Calorimetry}

Upon completion of shear-crystallization, approximately $7-10 \mathrm{mg}$ of sample was sealed hermetically in aluminum pans (PerkinElmer, Woodbridge, ON, Canada) and analyzed using a differential scanning calorimeter (DSC) (Pyris Diamond DSC, PerkinElmer, Woodbridge, ON, Canada). An empty aluminum pan was used as reference and the DSC was calibrated using an Indium standard. Samples were held isothermally at $20^{\circ} \mathrm{C}$ for $5 \mathrm{~min}$ and then heated to $90{ }^{\circ} \mathrm{C}$ at $10{ }^{\circ} \mathrm{C} / \mathrm{min}$. Thermograms were analyzed using Pyris Diamond DSC software (v.7, PerkinElmer, Woodbridge, ON, Canada).

\subsection{X-ray Diffraction}

Small (SAXD) and wide-angle (WAXD) X-ray diffraction of the fats was performed on a Hecus S3-MICROcaliX (Hecus X-ray Systems GmbH, Graz, Austria). The unit uses a low-power (50 W), high brilliance GeniX microfocus source and customized FOX-3D multilayer point focusing optics (Xenocs SA, Grenoble, France). The X-ray beam was generated by a $50 \mathrm{kV}, 1 \mathrm{~mA} \mathrm{Cu} \mathrm{K \alpha}$ anode. The spectra were captured using Hecus 1-D position-sensitive detectors (model PSD50M). An integrated calorimeter (Setaram Instrumentation, Caluire, France) allowed for in situ crystallization and melting of static samples. Approximately $20 \mu \mathrm{L}$ of melted sample were placed 
within 1.0 mm O.D. quartz capillary tubes (Charles Supper Company, Inc., Natick, MA, USA) using a $90 \mathrm{~mm}$ needle and syringe and characterized at room temperature. Sheared samples were analyzed at $25^{\circ} \mathrm{C}$ immediately following crystallization. Statically-cooled samples were subjected to a similar cooling protocol as sheared samples: held at $90^{\circ} \mathrm{C}$ for $15 \mathrm{~min}$, then cooled to $10{ }^{\circ} \mathrm{C}$ at $1{ }^{\circ} \mathrm{C} / \mathrm{min}$. Scans were collected each minute to capture the polymorphic form of the evolving system.

\subsection{Statistical Analysis}

All numerical data are reported as mean \pm standard deviation SD and were analyzed by one-way ANOVA. $\mathrm{P}<0.05$ was considered significant.

Table 1. Emulsion oil phase compositions and primary crystallization temperatures $\left(\mathrm{T}_{\mathrm{c}}\right)$.

\begin{tabular}{lcccc}
\hline \multicolumn{1}{c}{ Emulsifier } & Emulsifier $(\mathbf{w t} \%)$ & $\begin{array}{c}\text { FHCO } \\
(\mathbf{w t} \%)\end{array}$ & $\begin{array}{c}\mathbf{C O} \\
(\mathbf{w t} \%)\end{array}$ & $\begin{array}{c}\mathbf{T}_{\mathbf{c}} \\
\left({ }^{\circ} \mathbf{C}\right)\end{array}$ \\
\hline Control & 0 & 10.0 & 90.0 & $38.2 \pm 0.1$ \\
Glycerol monostearate (GMS) & 1.5 & 8.5 & 90.0 & $36.2 \pm 0.4$ \\
Glycerol monopalmitate (GMP) & 1.5 & 8.5 & 90.0 & $36.0 \pm 0.1$ \\
Glycerol monooleate (GMO) & 1.5 & 8.5 & 90.0 & $37.8 \pm 0.2$ \\
Sorbitan monostearate (SMS) & 1.5 & 8.5 & 90.0 & $37.4 \pm 0.3$ \\
Sorbitan tristearate (STS) & 1.5 & 8.5 & 90.0 & $35.9 \pm 0.1$ \\
Polyglycerol polyricinoleate (PGPR) & 0.25 & 10.0 & 89.75 & $37.8 \pm 0.7$ \\
\hline
\end{tabular}




\section{Results and Discussion}

\subsection{Viscosity Profiles}

The viscosity profiles of the shear-crystallized emulsions followed a three-phase sigmoidal pattern (Fig. 1) (Tran et al., 2014). Phase I was characterized by a slight increase in viscosity with decreasing temperature and resulted from enhanced interaction between triacylglycerol (TAG) molecules and/or emulsifiers in the molten state due to reduced thermal energy (DibildoxAlvarado et al., 2010; Tarabukina et al., 2009). Phase II, marked by the sharp increase in viscosity, was due to primary crystallization of the TAG and emulsifiers based on time-resolved x-ray diffraction studies of palm oil under shear by De Graef et al. and work by Mazzanti et al. (De Graef et al., 2009; Mazzanti et al., 2005). Phase III was visible as the continued, though reduced, increase in viscosity resulting from FHCO polymorphic transitions $\left(\alpha \rightarrow \beta^{\prime}\right.$ or $\left.\beta\right)$ and direct crystallization of the FHCO and emulsifiers likely into the $\beta^{\prime} / \beta$-forms (De Graef et al., 2009; Mazzanti et al., 2005). In a previous study, it was found that at a concentration of $1.5 \%(\mathrm{w} / \mathrm{w})$ the emulsifiers could co-crystallize with FHCO and be incorporated into its crystal lattice (Tran et al., 2015). This led to disrupted packing efficiency and resulted in shifts to lower crystallization temperatures compared to the control (Table 1). The emulsifiers that remained liquid at $20^{\circ} \mathrm{C}$ (PGPR and GMO) did not significantly affect crystallization temperature. The other two monoacylglycerides (MAGs), GMS and GMP, showed similar viscosity profiles, with an additional crystallization phase (phase IV) at $25.0 \pm 0.7{ }^{\circ} \mathrm{C}$ for GMS and $26.8 \pm 0.1{ }^{\circ} \mathrm{C}$ for GMP. The sorbitan-based emulsifiers, SMS and STS, also decreased primary crystallization temperatures with the STS having a greater effect due to its higher degree of molecular similarity to FHCO (three stearic acid residues) compared to SMS (one stearic acid residue) (Smith et al., 2011). 


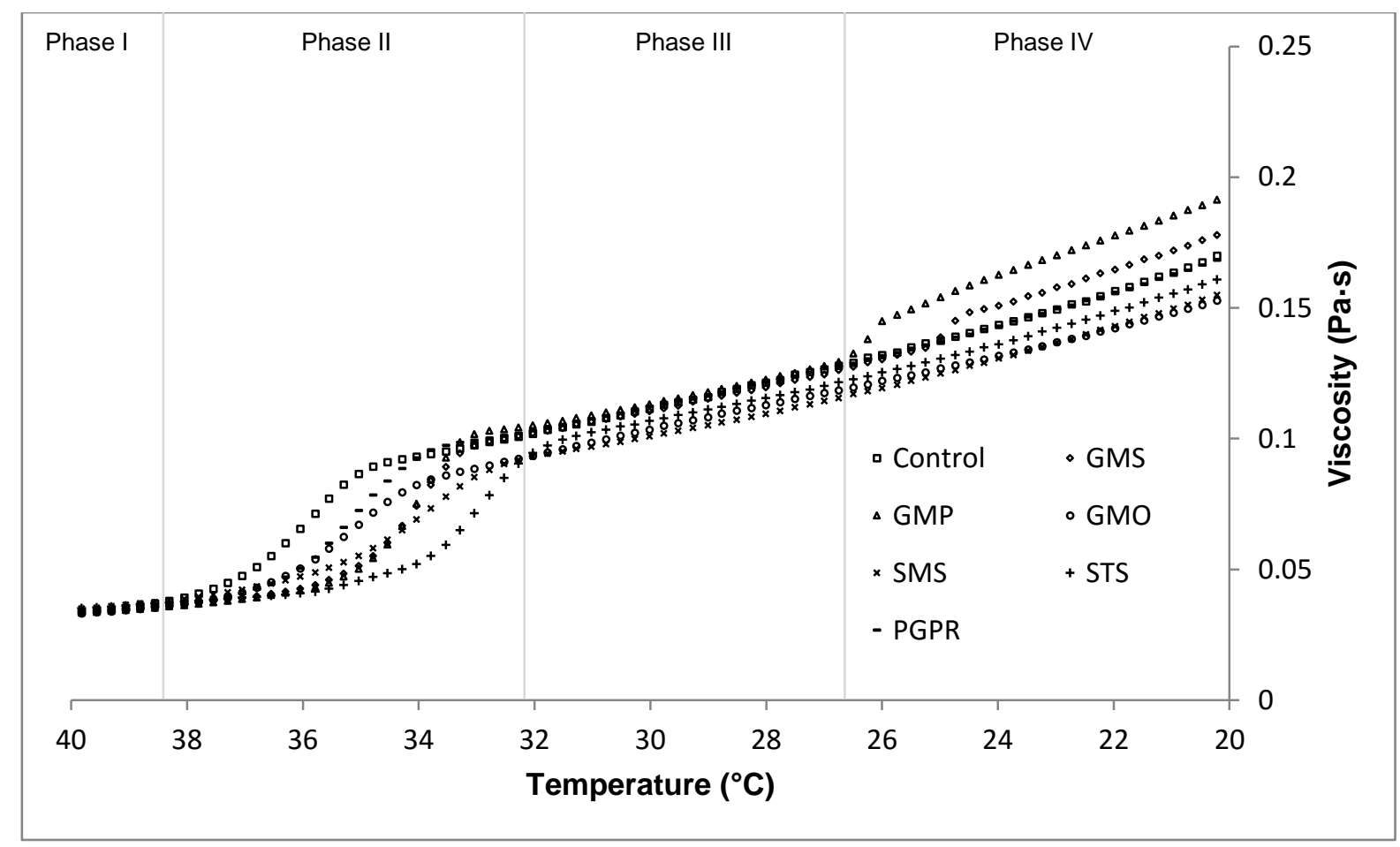

Figure 1. Viscosity profiles of sheared $10 \%(\mathrm{w} / \mathrm{w})$ water-in-oil emulsions using various emulsifiers $(n=3)$.

Comparison of the viscosity profiles of the sheared emulsions with their respective bulk oil phase blends showed that incorporation of a $10 \%(\mathrm{w} / \mathrm{w})$ dispersed aqueous phase did not have significant effects on crystallization temperatures, viscosity profiles, or final viscosity at $20{ }^{\circ} \mathrm{C}$, contrary to reports that the introduction of a dispersed phase tends to increase viscosity (data not shown) (Borwankar et al., 1992; Farah, Oliveira, Caldas, \& Rajagopal, 2005). This was probably because the action of shear caused the fat to crystallize into spheroids and spheroid-encapsulated droplets rather than a typical 3D network of sintered fat crystals with entrapped droplets (Ghosh et al., 2011). 


\subsection{Emulsion Microstructure}

The efficacy of Pickering droplet encapsulation within crystal spheroids was significantly impacted by emulsifier type. The control, not having any added emulsifier, showed mostly free water droplets and only a small number of encapsulated droplets, probably due to the limited emulsification capacity of the minor components in the FHCO (Fig. 2A). The MAGs showed higher encapsulation efficacy where the solid MAGs (GMS and GMP) showed uniform, highlycrystalline shells (Fig. 2B and C) whereas the GMO-based emulsions droplets' crystal shells appeared less-uniform, most likely due to the higher degree of encapsulation of smaller water droplets (Fig. 2D). There was also a difference in average encapsulated droplet size between the solid and liquid-state MAGs where GMS and GMP-based emulsions had an observed narrower size distribution of larger droplets while the GMO-based emulsions had a wider size distribution with many small droplets that were not encapsulated within crystal spheroid shells. Regardless of MAG type, there were many instances of multi-droplet encapsulation within irregularly-shaped crystal shells attributed to the crystallization of aggregates of spheroids during cooling (Fig. 2I). This was most apparent for GMS and GMP-based emulsions, where the solid MAGs have been shown to produce irregular shapes upon incorporation into the crystal matrix of FHCO (Tran et al., 2015). The high degree of crystallinity of GMS and GMP-based spheroids also caused some deformation of the water droplets which resulted in reduced droplet sphericity. It was also observed that GMO promoted the nucleation and crystal growth of FHCO spherulites at the oil-water interface of the droplets (Fig. 2H). This phenomenon was absent for the other emulsifiers and could be due to the kink in GMO's oleic residue delaying and disrupting the crystallization of FHCO, causing it to form spherulitic crystals rather than the highly-birefringent crystal shells as seen for GMS and GMP. 
The sorbitan-based emulsifiers showed poor encapsulation efficacy with incomplete crystal shell formation (Fig. 2E and F). The crystal shells of SMS-encapsulated droplets were large, irregularlyshaped, and took the shape of the aqueous cores, implying ineffective stabilization against coalescence compared to the solid-state MAGs, where droplets retained sphericity even when in encapsulated in close proximity (Fig. 2I). The crystal shells appeared to have a high degree of crystallinity but contained pores which allowed for the loss of aqueous droplets and/or the inward diffusion of CO (Fig. 2E red arrows). Smaller droplets could be seen within the water cores (Fig. 2E blue arrow) and could have been water droplets stabilized solely by SMS rather than SMSFHCO like the outer shells although this was unlikely due SMS' apparently poor ability to prevent coalescence. Irrespective, SMS was a poor emulsifier for the encapsulation of stable water droplets within crystal spheroid shells. Similarly, emulsions with STS also had poor crystal spheroid shell stability where the majority of shells were incompletely-formed (Fig. 2F). The water inside the crystal shells appeared crescent-shaped due to its tendency to minimize contact with the hydrophobic oil phase while maximizing its contact with the hydrophilic inner surfaces of the shells which were presumably lined with the sorbitan moieties of STS. Unlike the SMS emulsions, the crystal shells of STS emulsions were spherical, more uniform, and were thinner relative to the size of the droplets which suggested that although STS was effective at preventing coalescence during crystallization that would result in irregularly-shaped water cores, as seen for SMS, it lacked the mechanical strength to form complete shells due to its relative thinness. Based on its viscosity profile (Fig. 1), STS had the greatest effect on crystallization of FHCO due to its molecular similarity and propensity to stabilize the less-stable $\alpha$ polymorph which led to the formation of thinner and weaker Pickering crystal shells (Aronhime et al., 1987, 1988). PGPR, although highly effective at emulsifying and reducing droplet size, did not exhibit droplet 
encapsulation. We suspect this is due to the molecular structure of PGPR lacking complementarity with FHCO. The crystallizing FHCO was not able to template onto the ricinoleate chains of PGPR and thus was incapable of encapsulating the PGPR-stabilized emulsified droplets.

\section{A) Control}

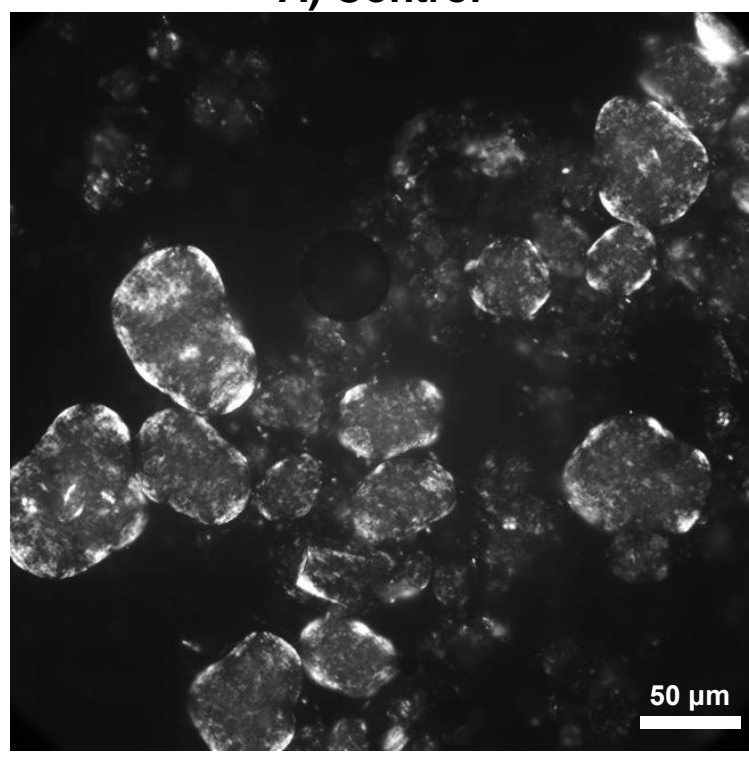

\section{C) GMP}

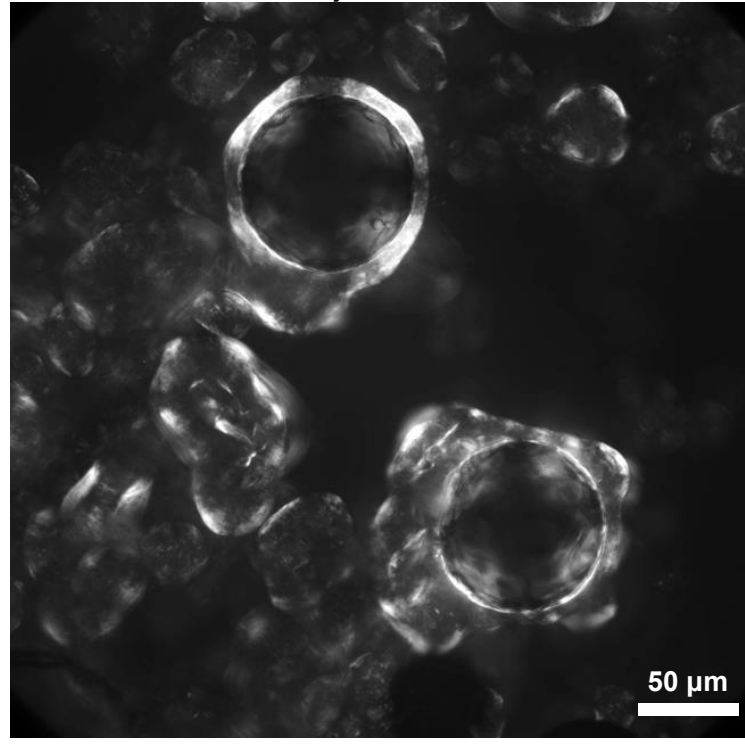

B) GMS

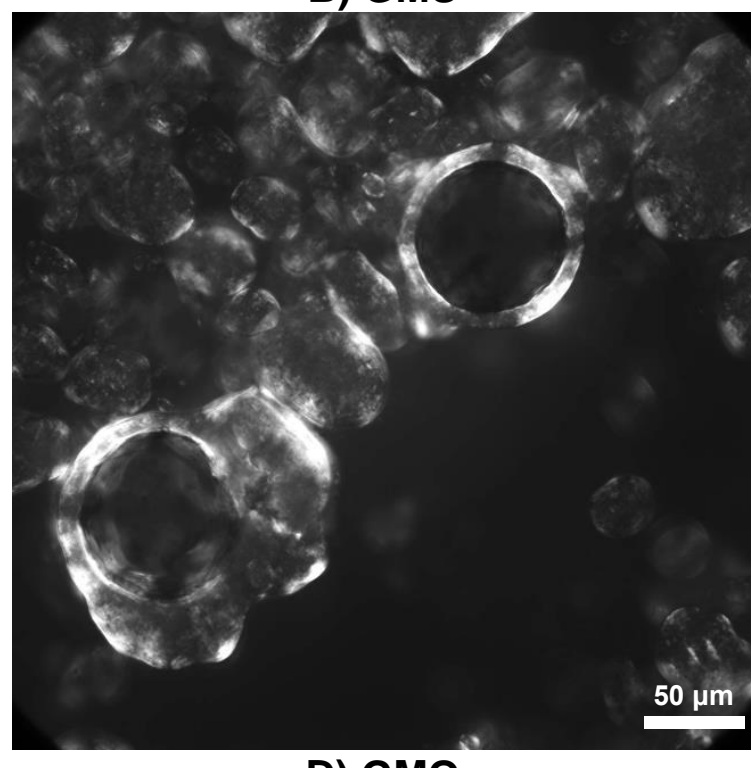

D) GMO

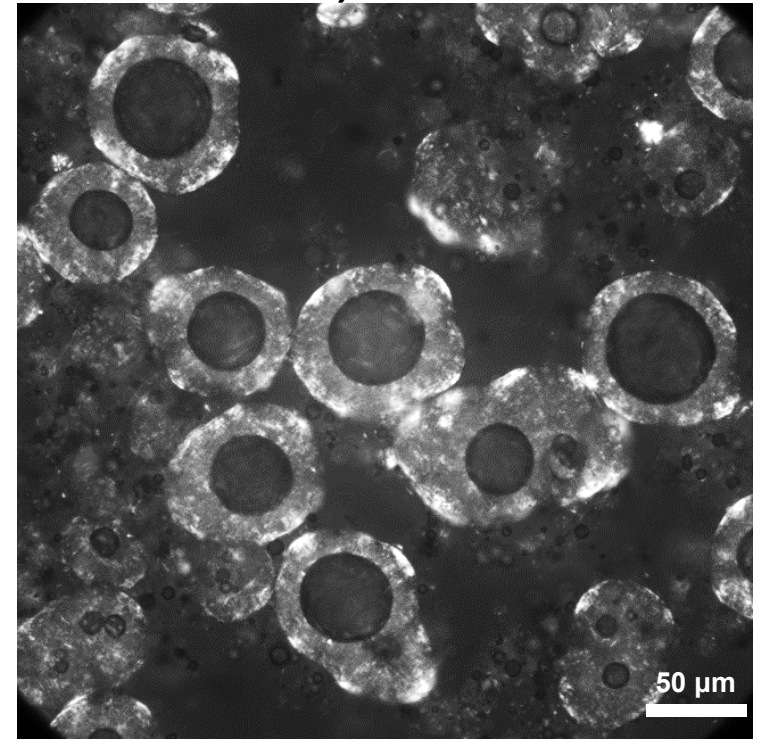


E) SMS

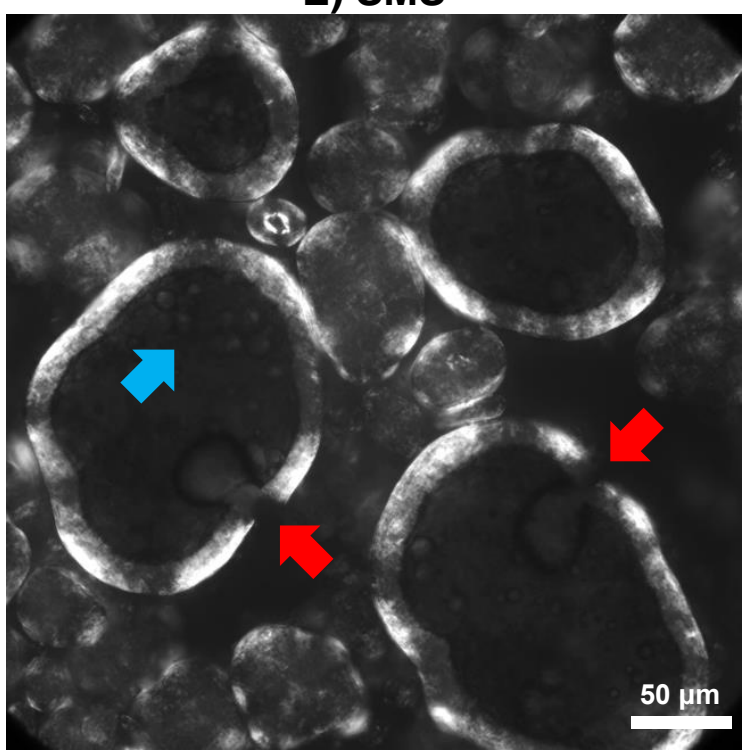

G) PGPR

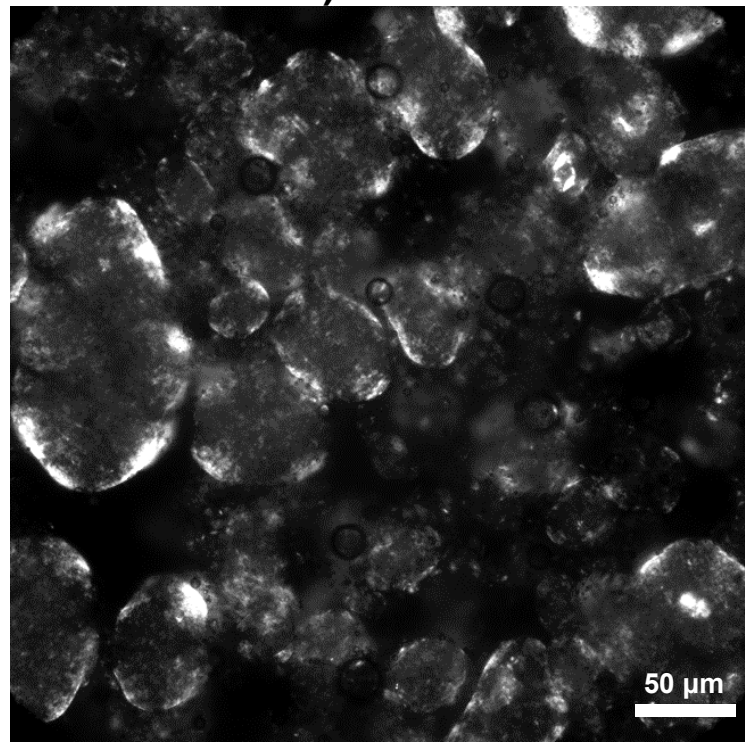

F) STS

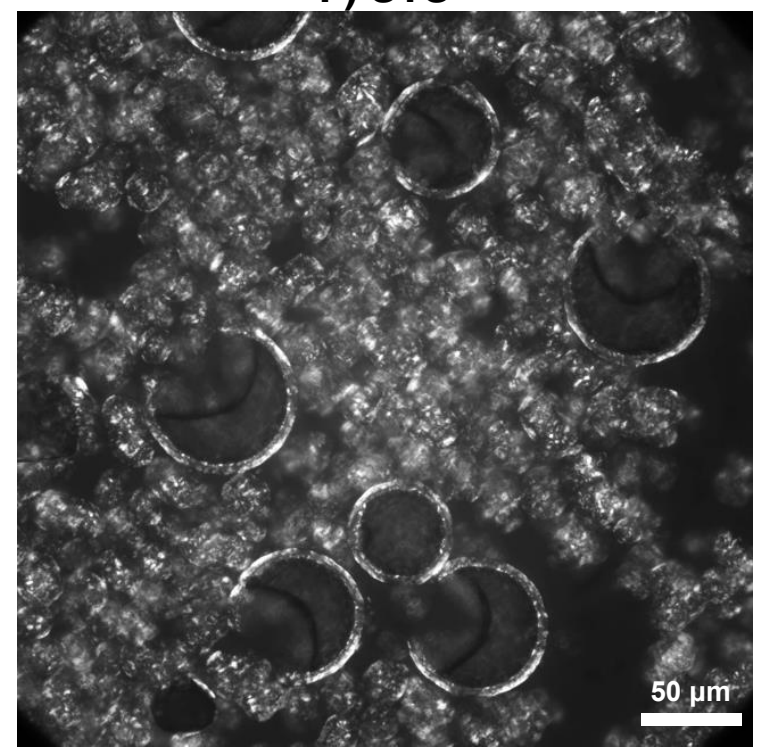

H) GMO-mediated interfacial crystallization of FHCO

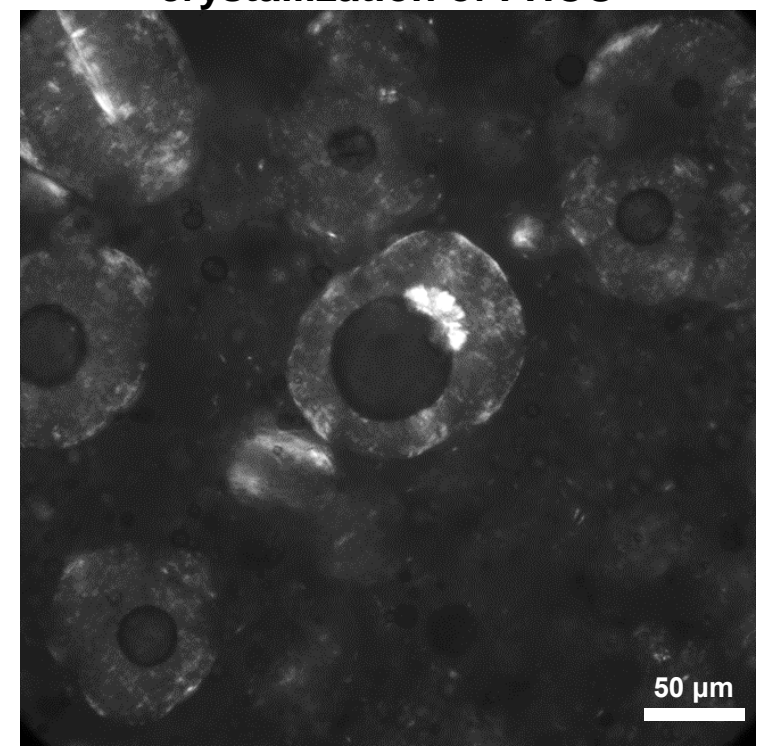




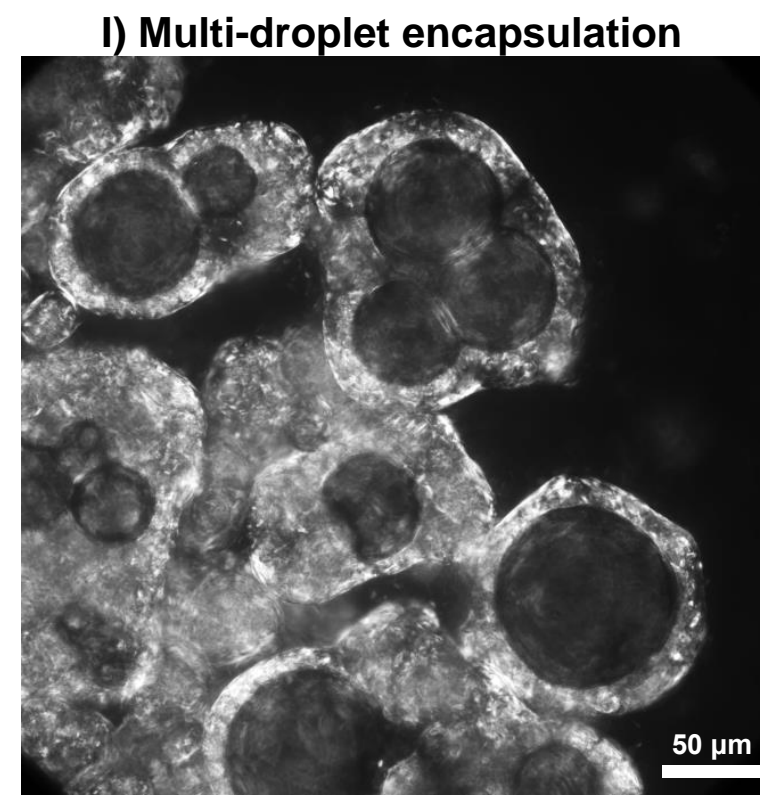

Figure 2. Microstructure of shear-crystallized 10\% (w/w) emulsions. Bar represents $50 \mu \mathrm{m}$.

\subsection{Thermal Analysis}

Thermal analysis of shear-crystallized bulk fat blends and their emulsion counterparts revealed that the introduction of $10 \%(\mathrm{w} / \mathrm{w})$ aqueous phase did not affect melting behaviour for systems prepared with liquid-state emulsifiers (GMO and PGPR) (Fig. 3). This indicated that there was little interaction between the emulsifiers and FHCO at the interface causing FHCO to preferentially crystallize separately in the continuous phase as spheroids due to shear, resulting in purer crystals with higher melting temperatures (Douaire et al., 2014). The solid-state emulsifiers (GMS, GMP, SMS, and STS) showed peak shifts to higher temperatures with the introduction of an aqueous phase which suggested the occurrence of emulsifier-mediated templating effects for FHCO crystallization at the droplets' oil-water interfaces. The greatest shifts in peak temperature were observed for the solid-state MAGs with shifts of $\sim{ }^{\circ} \mathrm{C}$ compared to shifts of $\sim 2{ }^{\circ} \mathrm{C}$ for the sorbitanbased SMS and STS which implied a greater templating effect by the glycerol-based emulsifiers, most likely because their polar moiety was identical to the glycerol-based TAGs in FHCO (Table 2). The larger sorbitan moiety in SMS and STS would more likely disrupt the packing efficiency 
of FHCO molecules thus resulting in reduced density and the stabilization of the $\alpha$ form while inhibiting polymorphic transformations to $\beta^{\prime} / \beta$, shown by the larger peaks of lower-melting fractions compared to the solid-state MAGs (Fig. 3). This would be a possible explanation for their ineffectiveness at crystal spheroid encapsulation observed via PLM, where the sorbitan-based emulsifiers showed incomplete shell formation (Fig. 2). These results are in line with findings by Wassell et al., who studied the effects of PGPR and monobehenoylglycerol (MB), a saturated MAG, on the crystallization behaviour of palm stearin-based W/O emulsions and found that MB promoted interfacial fat crystallization via templating effects while PGPR did not due to molecular dissimilarity (Wassell et al., 2012). Similarly, Shimamura et al. examined the effect of polyglycerine fatty acid esters (PGFEs) on the crystallization of palm stearin and found that PGFEs rich in palmitic acid moieties promoted interfacial crystallization of palm stearin while PGFEs containing oleic acid retarded it (Shimamura et al., 2013). The results of our study demonstrate that the type of emulsifier and the degree of molecular compatibility with the crystallizing phase may have significant effects on the efficacy of crystal spheroid shell formation and encapsulation of emulsion droplets. 


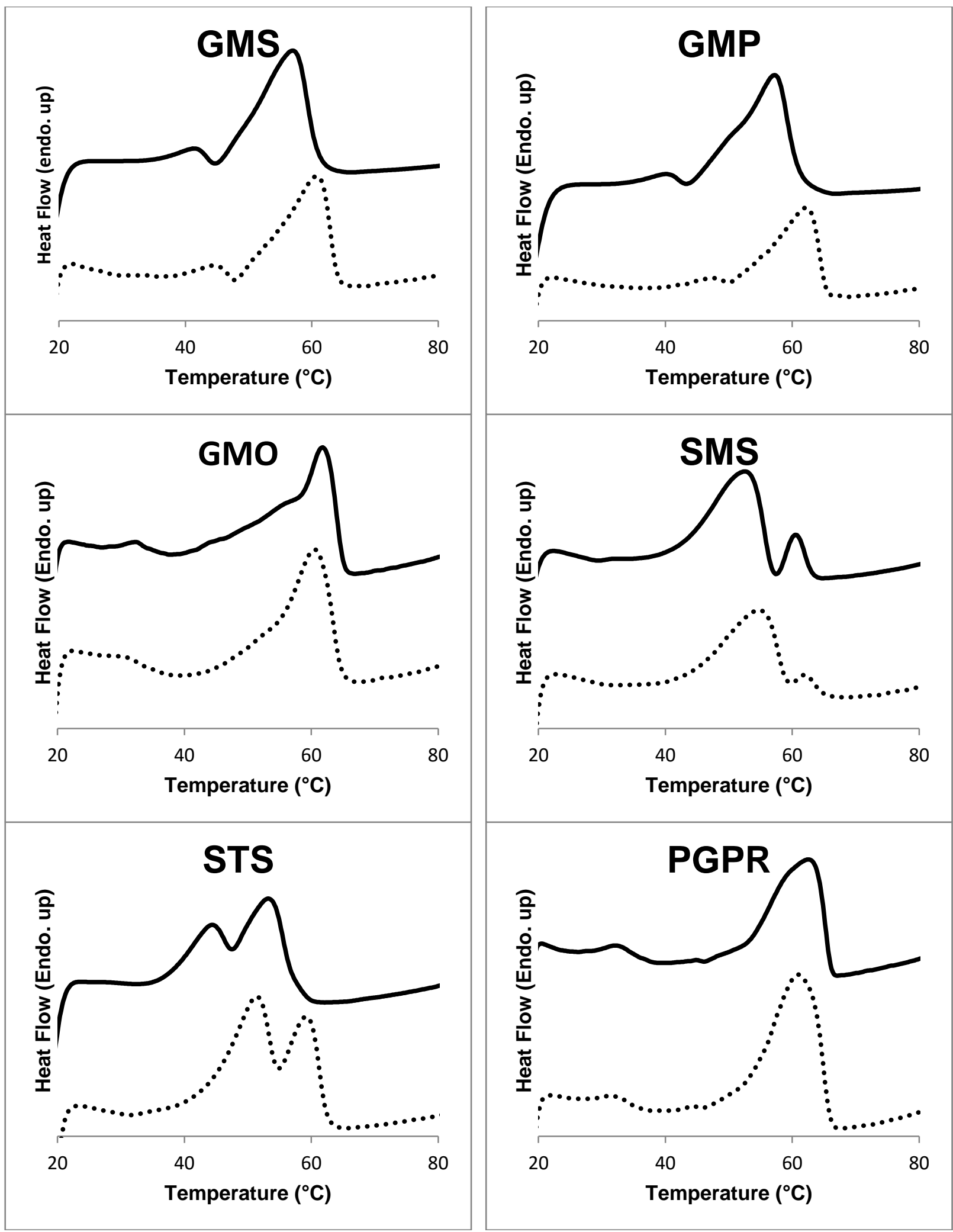

Figure 3. Differential scanning calorimetry melting thermograms of sheared oil phase blends (solid lines) vs. sheared 10\% (w/w) water-in-oil emulsions (dotted lines). 
Table 2. Peak melting temperatures for bulk FHCO-emulsifier blends and $10 \%$ (w/w) emulsions sheared at $1000 \mathrm{~s}^{-1}\left({ }^{\circ} \mathrm{C}\right)(\overline{\mathbf{X}} \pm \mathrm{SD})$.

\begin{tabular}{lll}
\hline & Bulk & Emulsion \\
\hline Control & $59.8 \pm 1.3,48.2 \pm 1.7$ & $62.1 \pm 1.7,51.9 \pm 1.5$ \\
GMS & $56.7 \pm 0.5,40.9 \pm 0.5$ & $61.2 \pm 0.4,43.4 \pm 0.0$ \\
GMP & $57.2 \pm 0.0,39.9 \pm 0.4$ & $61.5 \pm 0.5,46.5 \pm 0.3$ \\
GMO & $62.2 \pm 0.6,31.3 \pm 1.7$ & $60.4 \pm 0.1,29.9 \pm 1.6$ \\
SMS & $60.1 \pm 0.7,52.6 \pm 0.1$ & $62.3 \pm 0.3,52.3 \pm 1.3$ \\
STS & $54.7 \pm 1.1,45.3 \pm 1.4$ & $57.5 \pm 1.4,52.2 \pm 1.2$ \\
PGPR & $62.8 \pm 1.1,50.7 \pm 0.0$ & $61.7 \pm 1.0,30.6 \pm 1.4$ \\
\hline
\end{tabular}

\subsection{X-ray Diffraction}

X-ray diffraction measurements taken at $20^{\circ} \mathrm{C}$ revealed that, regardless of the presence or type of emulsifier, FHCO tended to crystallize mainly into the $\beta^{\prime}$ polymorph along with some $\alpha$-form (Fig. 4). This was similar to previous findings that incorporation of $1.5 \%(\mathrm{w} / \mathrm{w})$ emulsifier did not affect crystal spheroid polymorph (Tran et al., 2015). The (001) SAXD peak was located between $44-$ $48 \AA$ for all samples, indicating that TAGs were arranged in the $2 \mathrm{~L}$ chain length conformation. A general trend observed was a decrease in peak position as temperature increased, indicative of greater subcell ordering. The type of emulsifier had an effect on the resulting peak positions, provided that the emulsifier interacted with the crystallizing FHCO. PGPR consistently yielded similar peak locations and polymorphic forms to the control samples, which showed that it had little to no effect on FHCO crystallization. These results were in agreement with the thermal analysis data (Fig. 3) and microscopy (Fig. 2), where PGPR did not show encapsulation due to molecular dissimilarity, corroborating previous findings by Ghosh et al. (Ghosh \& Rousseau, 2012). The incorporation of bulkier emulsifiers (STS, SMS) caused SAXD peak locations to shift to higher values relative to the control and delayed $\beta^{\prime} \rightarrow \beta$ polymorphic transitions to higher temperatures which suggested reduced packing efficiency and stabilization of the $\alpha$ polymorph. 
The glycerol-based emulsifiers showed different behaviours between the liquid-state MAG (GMO) and solid-state MAGs (GMS and GMP), where GMO showed a similar SAXD peak location to those of PGPR and the control which indicated little emulsifier-FHCO interaction. In contrast, GMS and GMP exhibited relatively smaller shifts in long spacings compared to the sorbitan-based emulsifiers (SMS and STS) due to the smaller polar glycerol core relative to sorbitan.

Wide-angle x-ray diffraction produced weak signals given the low solids content. However, some general trends could be observed: the control emulsion, without any emulsifier, crystallized primarily into the $\beta^{\prime}$ polymorph with peaks at 4.21 and $3.81 \AA$ while the incorporation of emulsifier (except PGPR) resulted in shifts to larger spacings (Table 3). This indicated that the emulsifiers altered the crystal packing of the FHCO lamellae.

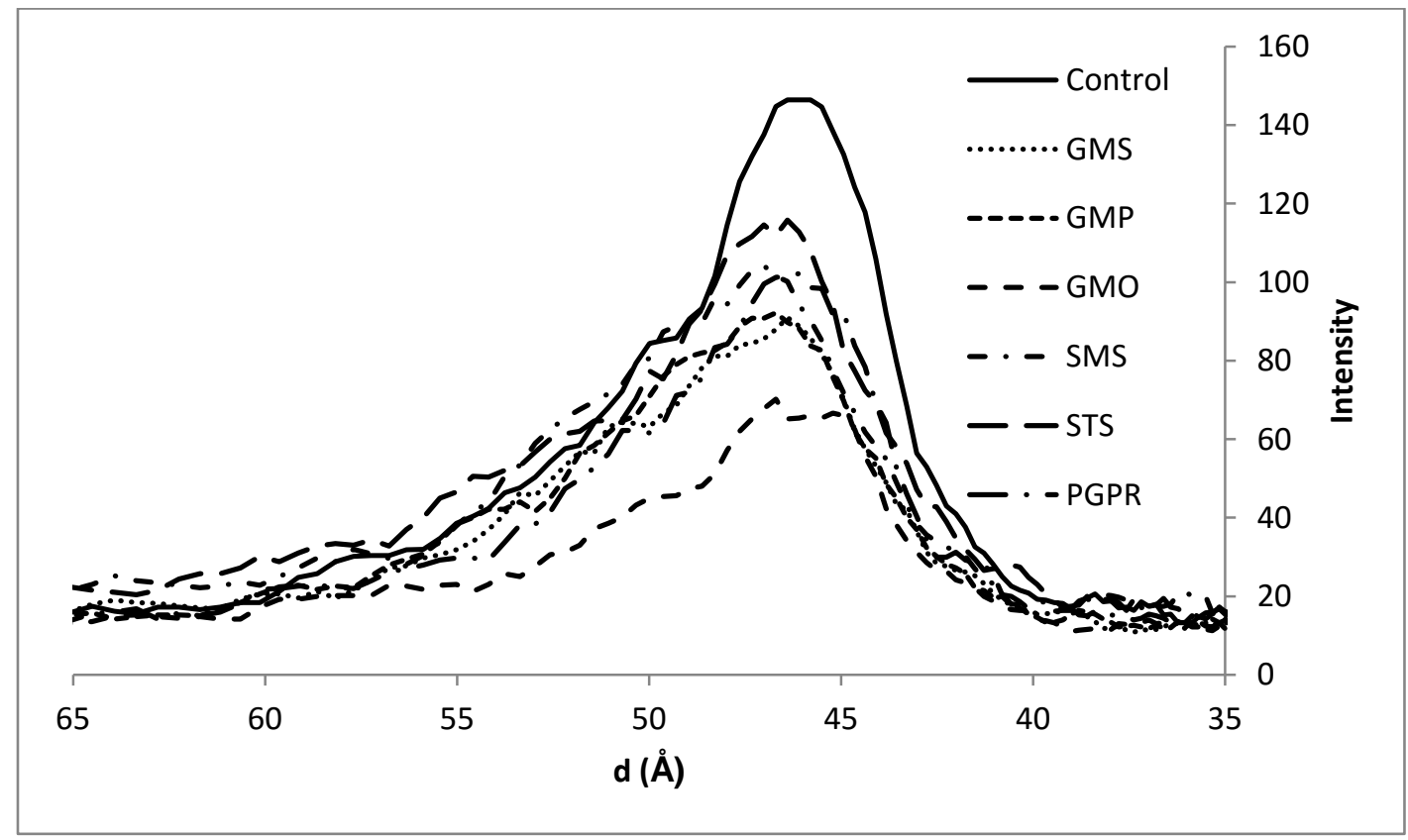

Figure 4. Small-angle x-ray diffraction of shear-crystallized emulsions at $20^{\circ} \mathrm{C}$. 
Table 3. Short and long spacings of shear-crystallized emulsions.

\begin{tabular}{ccc}
\hline Emulsion & Short spacings [WAXS] $(\AA)$ & Long spacings [SAXS] $(\AA)$ \\
\hline Control & $4.21,3.81$ & 45.8 \\
GMS & 4.25 & 46.4 \\
GMP & $4.3,4.22$ & 46.7 \\
GMO & 4.38 & 45.8 \\
SMS & $4.54,4.42,4.24$ & 47.0 \\
STS & $4.54,4.34,4.23$ (broad, weak) & 46.7 \\
PGPR & 4.21 (broad, weak), 3.81 & 46.4 \\
\hline
\end{tabular}

\subsection{Mechanism}

The proposed mechanism for laminar shear-induced crystal spheroid formation is multi-step: i) potential shear-induced pre-structuring of TAGs in the melt; ii) nucleation and formation of nanoplatelets (with relatively large distances between nanoplatelets and thus a low degree of interparticle interactions); iii) rotation and tumbling of platelets due to shear in the flow field; iv) nanoplatelet aggregation into extended stack-like structures; v) clustering of the nanoplatelet stacks into globular aggregates or bundles, depending on the shear rate; vi) crystallization into clusters of $\alpha$-crystals while tumbling, forming crystal spheroids; vii) shear acceleration of the $\alpha \rightarrow \beta^{\prime}$ (or $\beta$ ) polymorphic transition and finally viii) viscous heat generation causing melting of looselyassociated fat crystals at the spheroid surface, resulting in smoother surfaces (Tran et al., 2014). The presence and concentration of emulsifiers may have significant impacts on crystal spheroid formation and morphology, where a emulsifiers with a high degree of molecular similarity to the crystallizing fat, such as saturated MAGs, may act as seeds and surfaces for epitaxial TAG crystal growth whereas less-similar emulsifiers, such as sorbitan esters, may hinder spheroid formation by stabilizing lower metastable polymorphs (Tran et al., 2015). 
The introduction of an aqueous phase and thus an oil-water interface was shown to affect interfacial crystallization of a model fat (FHCO) where emulsifier similarity had significant effects on shear-induced crystal spheroid shell formation with both the polar moiety and non-polar chains playing important roles. When both the polar and non-polar groups were similar to the crystallizing fat, as for GMS and GMP, templating effects were observed and resulted in highly-ordered crystalline shells. If the emulsifier's fatty acid (FA) residue was different to the fat, as for GMO with its bent chain, templating effects were reduced and crystallization of the fat was delayed. Differences in polar moiety had greater impact on crystal spheroid shell formation than FA chain similarity, as shown for SMS and STS, where templating effects were observed but crystal shell integrity was compromised. When both the polar and non-polar moieties were dissimilar to the fat, as for PGPR, no templating effects were observed. 


\section{Conclusion}

Shear-crystallization of a bulk fat blend within a narrow gap may produce spheroidal crystals. The microstructure and physical properties of these crystal spheroids can be tailored via selection of processing conditions, such as shear and cooling rates, or by the addition of emulsifiers.

Results from the present study suggest that for crystal spheroid droplet encapsulation to occur, the emulsifier must be molecularly-compatible with the fat in order to promote interfacial crystallization via templating effects. The liquid-state emulsifiers (GMO and PGPR) hindered crystallization at the oil-water interface, where GMO delayed crystallization while PGPR prevented fat-emulsifier interactions and instead promoted crystallization in the continuous oil phase. The solid-state emulsifiers showed templating effects with the MAGs being more effective than the sorbitan esters. Encapsulation efficacy could be further tuned by studying the effect of the emulsification process, to assess whether initial droplet size affects crystal spheroid shell formation, the effect of volume fraction of dispersed phase and/or emulsifier concentration, or by varying cooling and shear rates. Further work is required to better-understand the effects of shearcrystallization on Pickering encapsulation. 


\section{Chapter 5 - The Use of Shear to Produce Novel Crystal Microstructures}

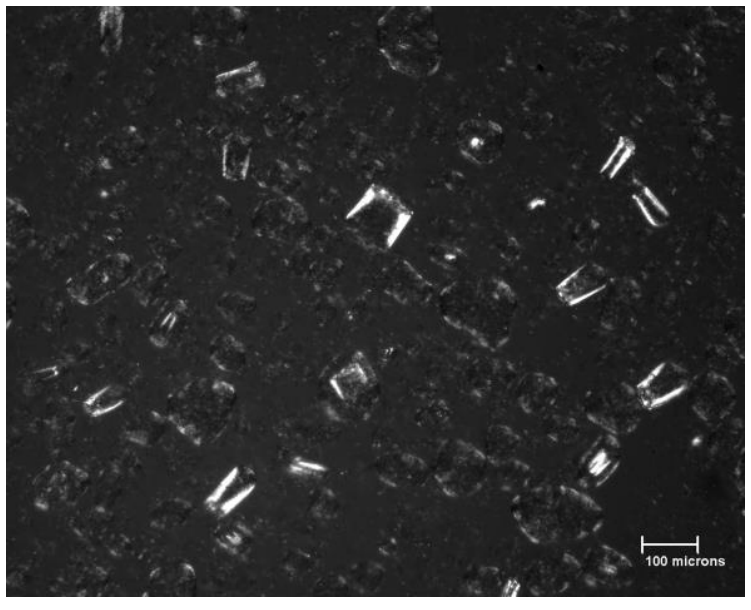

- Laminar shear may be used to produce spheroidal and cylindrical crystal structures

- Mechanisms proposed for their formation 


\begin{abstract}
A model fat system composed of $10 \%(w / w)$ fully-hydrogenated canola oil (FHCO) in canola oil (CO) was subjected to shear-crystallization within the confined gap between two parallel plates of a rheometer. The resulting spheroidal and cylindrical crystalline assemblies were characterized via polarized light microscopy (PLM). The formation of fat crystal spheroids and cylinders was significantly affected by shear rate with higher shear rates producing more numerous, smaller spheroids but fewer crystal cylinders with smaller diameters. A "log-rolling" crystallization mechanism was proposed for the formation of the observed fat crystal cylinders. These results demonstrated that shear-crystallization within a confined gap may produce novel cylindrical microstructures.
\end{abstract}




\section{Introduction}

Fats, which are typically blends of triacylglycerols (TAGs), are employed in many industries such as the pharmaceutical, cosmetics, and food industries usually as the chassis for products or as carriers for the delivery of target compounds (Sato, 2001). Their mechanical properties (e.g. texture, firmness, and spreadability), physical stability (e.g. phase separation), and organoleptic properties (e.g. mouthfeel or meltability) depend on the underlying structure of the fat crystals and their interactions which typically results in three-dimensional networks of sintered crystals. The composition of the fat (i.e. types and distributions of TAGs along with minor components such as emulsifiers (e.g. mono- and diglycerides) or non-fat ingredients may impact crystallization behaviour (Smith et al., 2011). Processing conditions such as cooling rate, crystallization temperature, and mixing/agitation (shear) may also affect crystallization and result in different crystal types, known as polymorphism. The three main polymorphs that TAGs form, in order of increasing molecular organization, density, and stability, are $\alpha, \beta^{\prime}$, and $\beta$. Faster cooling rates and/or lower crystallization temperatures typically result in the formation of the $\alpha$ polymorph while slower cooling and/or higher crystallization temperatures result in the more stable $\beta^{\prime}$ and/or $\beta$ polymorph(s). Once formed, lower polymorphs may undergo transformations to higher polymorphs via molecular arrangements usually by way of a liquid phase $\quad$ (e.g. $\alpha \rightarrow \beta^{\prime}$ $\rightarrow \beta$ ). Polymorphic transitions from higher-to-lower polymorphs never occur (Hartel, 2001; Marangoni \& Wesdorp, 2013; Walstra, 2003).

Recently there has been increased interest in understanding the role of shear on fat crystallization. Shear is often used in industry to improve mass and heat transfer resulting in improved product homogeneity (Campos \& Marangoni, 2014; Stapley et al., 1999). Shear may enhance TAG nucleation by providing enough energy to overcome activation energy barriers (Hartel, 2001) 
and/or by breaking down larger crystals into fragments which may act as seed surfaces for further nucleation (Kloek et al., 2005; Maleky \& Marangoni, 2008). This ordinarily leads to enhanced crystallization and crystal networks with increased mechanical strength (Marangoni \& Narine, 2002; Suresh S. Narine \& Marangoni, 1999).

Laminar shear flow, which may be generated via Couette-type shear cells (rotating concentric cylinder apparatuses) (Maleky \& Marangoni, 2008) or between a system of parallel plates, such as those of a rheometer (Ghosh \& Rousseau, 2012), has been shown to affect fat crystallization at the nanoscale, impacting the size and morphology of resulting crystal nanoplatelets (Maleky et al., 2012, 2011). Laminar shear may also cause fat crystals to orient themselves in the direction of shear flow, with results depending on crystal size and shear rate (Mazzanti et al., 2003). Recent studies have shown that laminar shear, above a critical rate, may shape crystallizing fats into spheroidal microstructures (Acevedo et al., 2012; Ghosh \& Rousseau, 2012). In an effort to elucidate the processing conditions responsible for the formation of these spheroids, Tran et al. studied the crystallization of a model fat system [a blend of fully-hydrogenated canola oil (FHCO) and canola oil (CO)] under various shearing and cooling conditions and proposed a mechanism for their formation (Tran et al., 2014). In this study we expand on the role of shear on fat crystallization, characterize a novel cylindrical crystal microstructure, and propose a mechanism of formation. 


\section{Materials and Methods}

\subsection{Materials}

Canola oil (CO) (acid value $\sim 0.2 \%$ ) was purchased from a local grocery store and stored at room temperature until use. Fully-hydrogenated canola oil (FHCO) was purchased from Bunge (Oakville, ON, Canada) and had a capillary melting point of $69.5^{\circ} \mathrm{C}$ (AOCS official method Cc $1-25$ ) and a free fatty acid content of $0.018 \%$ (AOCS Society, 1998). The predominant TAG species in $\mathrm{FHCO}$ were $\mathrm{C}_{52}(13.3 \%), \mathrm{C}_{54}(73.6 \%)$, and $\mathrm{C}_{56}(5.0 \%)$.

\subsection{Shear-Crystallization}

FHCO was mixed with CO to produce a $10 \%(\mathrm{w} / \mathrm{w})$ blend and then heated to $70{ }^{\circ} \mathrm{C}$ with continuous stirring for at least 30 minutes to ensure complete melting of the fat. Aliquots were then deposited between the parallel plates (PP25, $25 \mathrm{~mm}$ diameter, $0.5 \mathrm{~mm}$ gap) of a rheometer (Physica MCR301, Anton Paar, Ville St-Laurent, QC, Canada). Samples were maintained at $70{ }^{\circ} \mathrm{C}$ for 20 min and then cooled at $1.0^{\circ} \mathrm{C} / \mathrm{min}$ while being sheared at a constant rate ranging from $0-2000$ $\mathrm{s}^{-1}$. The apparent viscosity of the samples was measured using the rheometer and measurements were recorded as samples were sheared and cooled. All experiments were performed at least in triplicate.

\subsection{Microscopy}

The microstructure of samples was observed via polarized light microscopy (PLM). Samples were placed on viewing slides at room temperature $\left(\sim 25^{\circ} \mathrm{C}\right)$ (Fisher Scientific, Ottawa, ON, Canada), covered with a cover slip (Fisher Scientific, Nepean, ON, Canada) and analyzed with an inverted light microscope (Axiovert 200M, Zeiss Inc., Toronto, ON, Canada) equipped with a CCD camera (Q Imaging, RETIGA 32-0012B-105, Surrey, BC, Canada) using QImaging Q-Capture Pro 7 software (Version 7.0.5 Build 4325, QImaging, Inc., Surrey, BC, Canada). 


\section{Results and Discussion}

The shear-crystallization of FHCO-CO blends resulted in the formation of mostly spheroidal fat crystal microstructures (Fig. 1). The size and morphology of these crystal spheroids were dependent on both cooling and shear rates with shear having greater impact. Higher shear rates resulted in decreased spheroid sizes and increased sphericity. A mechanism for their formation has been proposed in a previous study (Tran et al., 2014). To recap, the formation of spheroidal fat crystals is multi-step: i) potential shear-induced pre-structuring of TAG in the melt; nucleation and formation of nanoplatelets and subsequent CNP stacks; iii) orientation of CNP stacks parallel to the shear flow field below a critical shear rate or above which tumbling and rotation of nanoplatelet stacks occured; iv) crystallization into bundles or clusters of $\alpha$ crystals; v) shear-accelerated polymorphic transitions to $\beta^{\prime} / \beta$; and vi) smoothening of spheroid surfaces due to viscous heat generation (Fig. 2). 

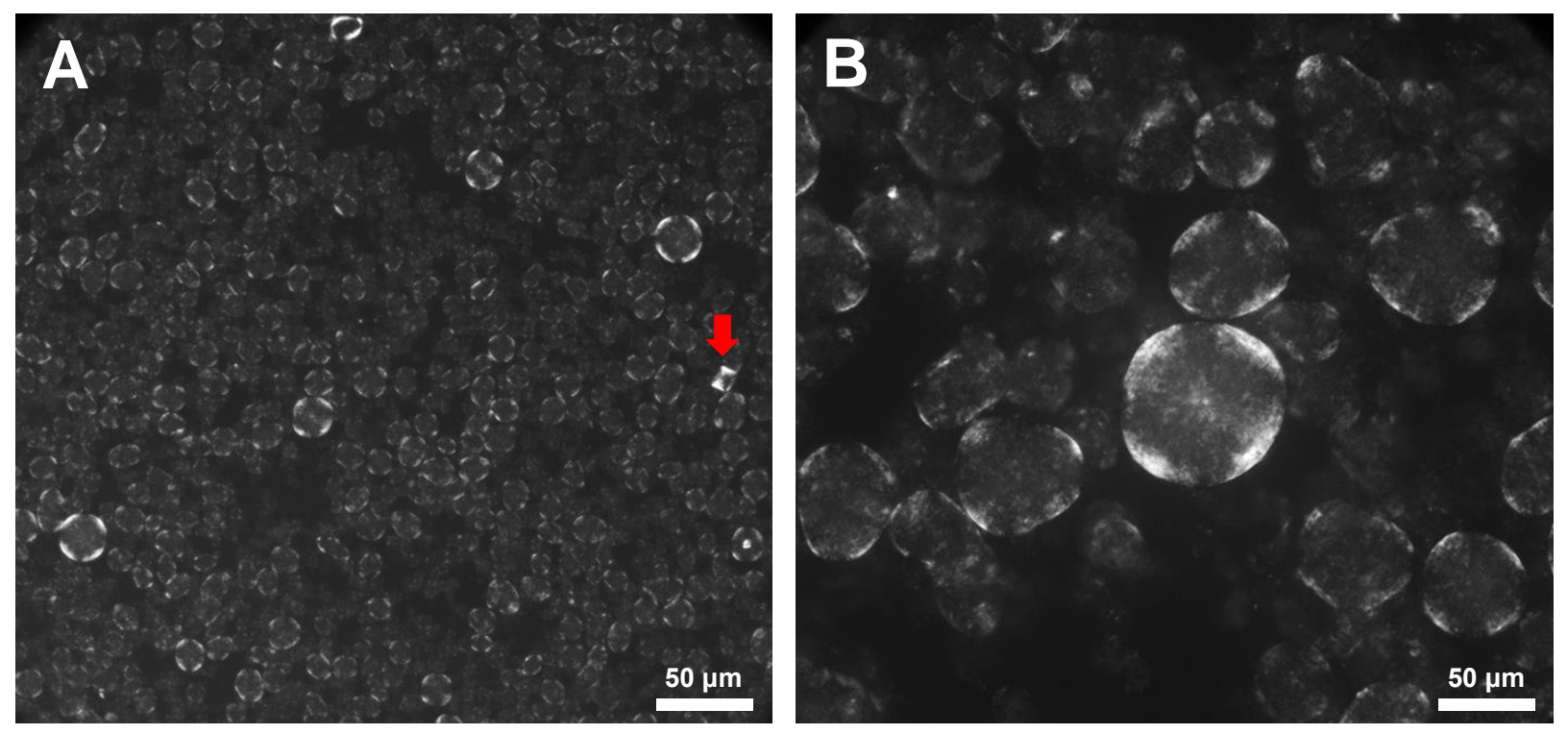

Figure 1. PLM image of fat crystal spheroids formed via the shear-crystallization of $10 \%(\mathrm{w} / \mathrm{w})$ FHCO-CO blends at a cooling rate of $1.0{ }^{\circ} \mathrm{C} / \mathrm{min}$ and a shear rate of $2000 \mathrm{~s}^{-1}$. A) $100 \mathrm{X}$ magnification where the bar represents $100 \mu \mathrm{m}$. B) 400X magnification where the bar represents $50 \mu \mathrm{m}$.

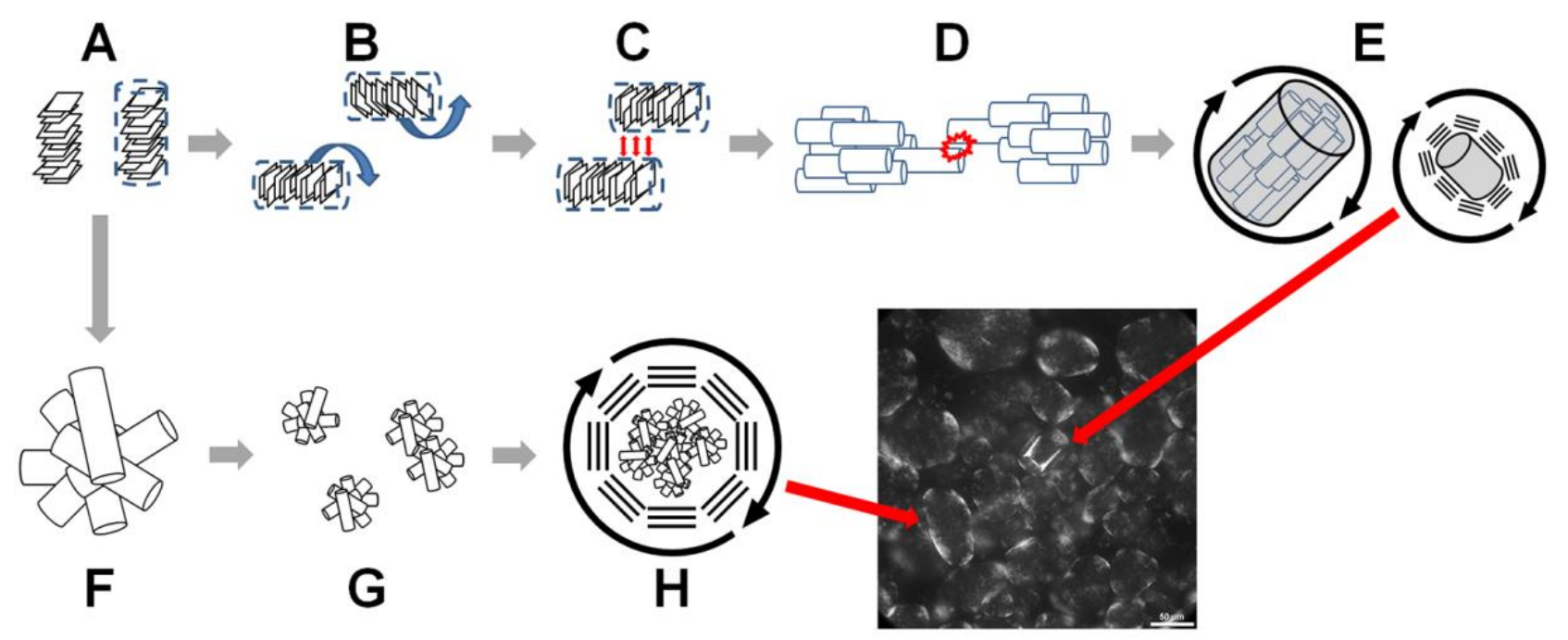

Figure 2. Proposed mechanism for the shear-crystallization formation of crystal spheroids and cylinders. 
In addition to spheroidal fat crystals, shear-crystallization of the fat within the narrow gap of a parallel plate geometry also resulted in the formation of cylindrical crystalline assemblies (Fig. 1 red arrow and Fig. 3). These crystal cylinders represented only a small fraction of the total number of crystal assemblies and varied in both diameter size and height. Some of these structures were cylindrical, with two faces of approximately the same diameter, while most were shaped more like conical frustums, with one face having a smaller diameter than the other. Additionally, the wider ends of these frustum-shaped assembles were usually rounded (highlighted by arrows in Fig. 3A). The surfaces of these crystal cylinders/conical frustums displayed high brightness intensities under polarized light which indicated a high degree of crystallinity most likely of $\beta$ ' or $\beta$ polymorph. The edges of the flat faces were sometimes concave in shape (Fig. 3B) and other times flat with welldefined edges (Fig. 3C). These cylinders exhibited a distribution of sizes for a given shear rate and represented only a small fraction of crystalline assemblies, where most of the fat crystallized into spheroids, with an increased number of cylinders/frustums observed at lower shear rates. The minimum shear rate threshold for the formation of these crystal cylinders/frustums was $400 \mathrm{~s}^{-1}$. Increased shear rates resulted in fewer cylinders formed and decreased diameters due to reduced contact times between TAG molecules at the crystal interface resulting in inhibition of crystal growth. The proposed mechanism for the formation of these cylinders/frustums follows the same pathway outlined in Figure 2 (A to E) but without further epitaxial growth that would result in spheroidal crystal shells. Based on the high degree of surface crystallinity observed, perhaps the degree of TAG molecular orientation required for successful surface integration was higher for cylindrical growth compared to spheroidal growth, which led to relatively fewer cylinders formed. 

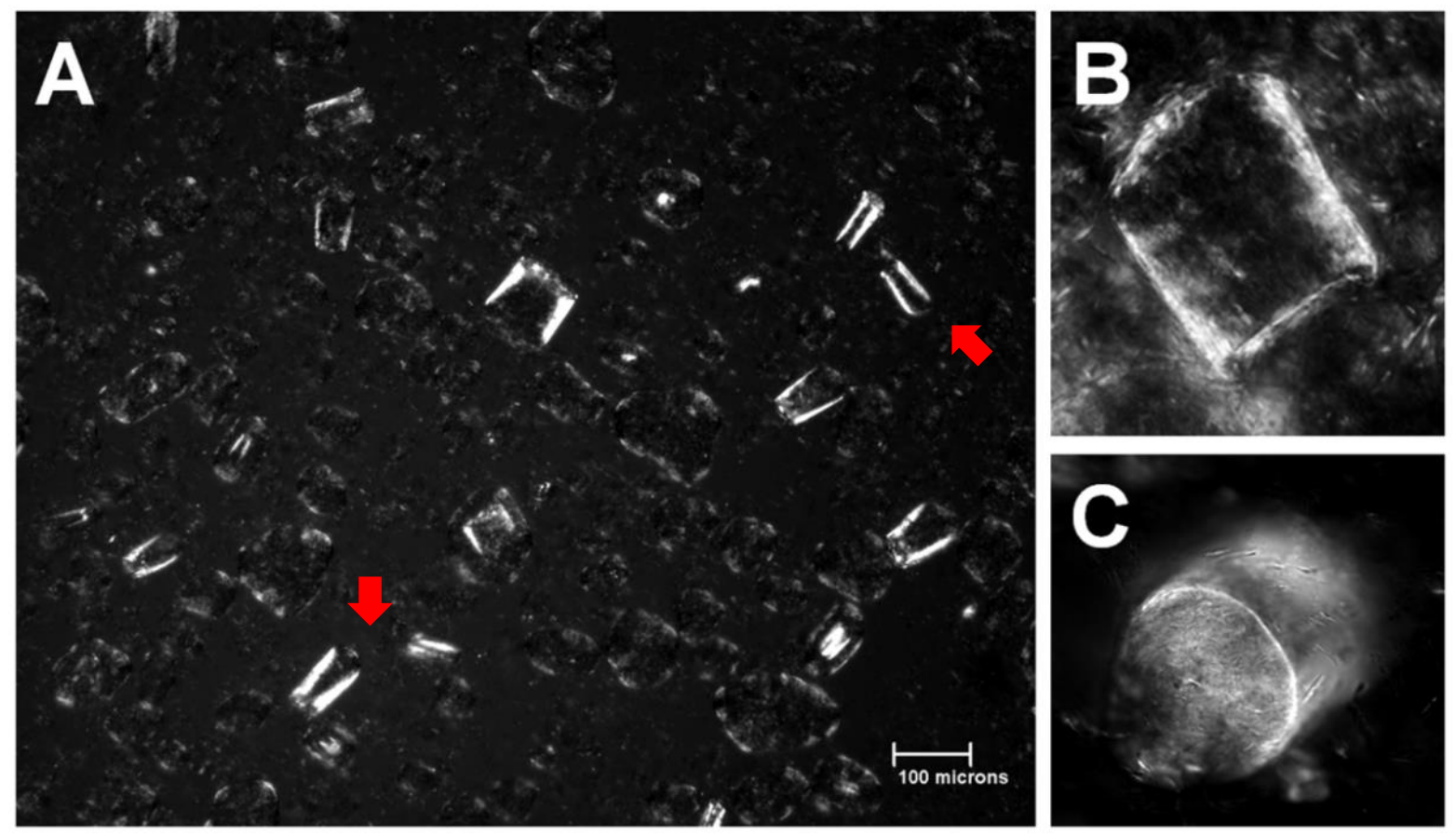

Figure 3. PLM images of fat crystal cylinders formed via the shear-crystallization of $10 \%(\mathrm{w} / \mathrm{w})$ FHCO-CO blends.

An alternate mechanism for cylinder formation could be a "log-rolling" action induced by the laminar shear, with the cylinders being oriented perpendicularly to the direction of shear flow forming from crystallizing fat trapped within vortices in the flow field (Fig. 4). The frustum-like structures could be a product of the radial velocity gradients generated by the parallel plate geometry caused by the high rotational velocity of the plate edge relative to the center. 


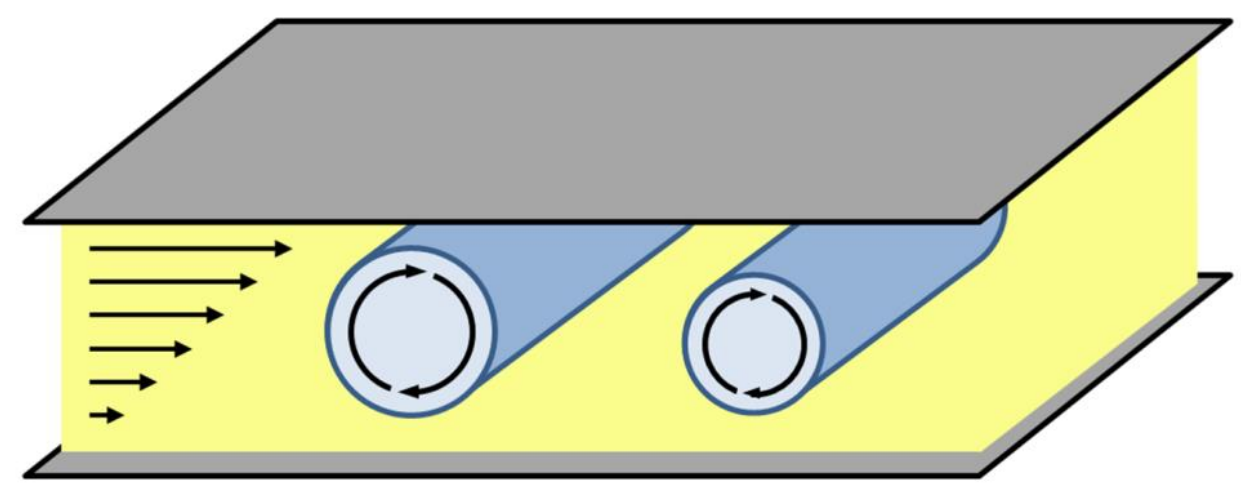

Figure 4. Schematic of "log-rolling" crystallization mechanism.

A "log-rolling" flow-alignment regime has been observed for the crystallization of liquid crystalline systems when shear was below a critical rate (Arya \& Panagiotopoulos, 2005; Hao et al., 2010; Li et al., 2014; RomoUribe \& Windle, 1996). Romo-Uribe and Windle studied the alignment of main-chain liquid polymer melts (1,4-hydroxybenzoic acid and 2,6hydroxynapthoic acid) under rotational shear and found that below a critical shear rate $\quad\left(10 \mathrm{~s}^{-}\right.$ $\left.{ }^{1}\right)$, a log-rolling regime was observed, where the polymers aligned with the vorticity axis normal to the shear-flow (RomoUribe \& Windle, 1996). Additionally, they observed that low molecular weight polymers $(4600 \mathrm{~g} / \mathrm{mol})$ were more susceptible to the log-rolling alignment while larger molecules tended to align in the direction of shear flow. It would therefore be a reasonable assumption that tristearin, the main crystallizing TAG component of FHCO $\quad(\sim 890 \mathrm{~g} / \mathrm{mol})$, could be susceptible to a log-rolling regime. Arya and Panagiotopoulos reported similar findings for molecular dynamics simulations of amphiphilic molecules under shear in a confined gap, where it was shown that the molecules formed cylindrical micelles that oriented themselves perpendicular to the shearing direction, rolling about their axes via a log-rolling regime (Arya \& 
Panagiotopoulos, 2005). Further work examining the structuring-effects of shear and the optimization of processing conditions for the formation of these crystal cylinders is required.

\section{Conclusion}

Shear may have significant impacts on fat crystallization, affecting processes from the nano- to the mesoscale. Above a critical rate, shear may induce the tumbling of fat crystals in the flow field, resulting in the formation of spheroidal morphologies. Cylindrical crystalline assemblies may also be formed, possibly via a "log-rolling" mechanism where the crystallizing fat becomes trapped within vortices perpendicular to the flow field causing them to roll along their axes. The structuring-effects of shear on fat crystallization are still not fully understood and further work is required for its elucidation. 


\section{General Conclusions and Future Work}

Processing conditions may have significant effects on fat crystallization and the resulting crystal microstructures. Laminar shear can be used to induce the formation of spheroidal and cylindrical crystalline assemblies whose properties depend on shear and cooling rates. Higher shear rates resulted in increased crystal sphericity and decreased average crystal spheroid and cylinder size due to breakage of larger crystals and decreased interaction times between TAG molecules in the melt and crystal surfaces. Lower shear rates promoted interaction between TAG molecules and resulted in larger, more irregularly-shaped crystal spheroids. Crystal spheroid morphology may be further controlled by crystallizing at an optimal cooling rate. The presence of emulsifiers significantly affects the formation and morphology of crystal spheroids with effects being most substantial when there is a high degree of molecular similarity between the emulsifier and the crystallizing fat. The crystal spheroids have potential for the encapsulation of dispersed emulsion droplets where the efficacy of Pickering-type crystal shell formation depends on compatibility between the emulsifier and fat. Emulsifiers with molecular structures significantly different from the crystallizing fat may delay or even prevent interfacial crystallization and thus spheroidal crystal shell formation while molecularly-similar emulsifiers may promote interfacial crystallization via templating effects, resulting in crystal spheroid-encapsulated droplets.

The effects of shear on fat crystallization are still not fully understood and more research is required for elucidation. Possible areas for further research may include using time-resolved $x-$ ray diffraction during shearing to observe polymorphism and transformations during crystallization. Synchrotron radiation x-ray diffraction may be a suitable technique for the highresolution characterization of crystal spheroid polymorphism and possible shear-induced orientation effects within the crystal spheroids (i.e. is there a difference in crystal 
arrangement/polymorph between the center of the spheroids and the outer shells? Does the tumbling action during crystallization produce radially-organized crystal lamellae? How does fat crystal organization at the nano- and/or mesoscale affect the Maltese cross birefringence patterns observed?). Cryo-transmission electron microscopy may be used to image crystal lamellae at the nanoscale to provide insight on molecular organization within the crystal spheroids and cylinders. Further work on the effects of emulsifiers and their use in crystal spheroid-based encapsulation of emulsion droplets may be expanded to different types and concentrations of emulsifiers, the effect of droplet size and volume fraction of dispersed phase, or emulsification regime (e.g. high-pressure valve homogenization vs shear produced via a rotor-stator). In contrast to studying the effect of emulsifiers and minor components, one could study and compare the shear-crystallization of pure systems consisting of only one type TAG (i.e. are minor components required for crystal spheroid formation? Are there specific TAG properties such as chain length or saturation/unsaturation that are required?). Additionally, target molecules could be loaded into the spheroid-encapsulated droplets and their release conditions and profiles could be characterized. The crystal spheroids could possibly be incorporated into food, cosmetic, or pharmaceutical products to assess their effects on rheological and organoleptic properties such as viscosity and mouthfeel, respectively. Because these fat crystal microstructures have only recently been discovered and characterized, there is great potential for further research. 


\section{References}

Acevedo, N. C., Block, J. M., \& Marangoni, A. G. (2012). Critical laminar shear-tempearture effects on the nano- and mesoscale structure of a model fat and its relationship to oil binding and rheological properties. Faraday Discuss., 158, 171-194.

Acevedo, N. C., \& Marangoni, A. G. (2010a). Characterization of the nanoscale in triacylglycerol crystal networks. Crystal Growth \& Design, 10(8), 3327-3333.

Acevedo, N. C., \& Marangoni, A. G. (2010b). Toward nanoscale engineering of triacylglycerol crystal networks. Crystal Growth and Design, 10(8), 3334-3339. http://doi.org/10.1021/cg100469x

Acevedo, N. C., \& Marangoni, A. G. (2014a). Engineering the functionality of blends of fully hydrogenated and non-hydrogenated soybean oil by addition of emulsifiers. Food Biophysics, 9, 368-379.

Acevedo, N. C., \& Marangoni, A. G. (2014b). Functionalization of non-interesterified mixtures of fully hydrogenated fats using shear processing. Food Bioprocess Technol., 7, 575-587.

Acevedo, N. C., Peyronel, F., \& Marangoni, A. G. (2011). Nanoscale structure intercrystalline interactions in fat crystal networks. Current Opinion in Colloid and Interface Science, 16(5), 374-383. http://doi.org/10.1016/j.cocis.2011.05.004

AOCS. (1991). Introduction to fats and oils technology. New Orleans, LA, USA: AOCS Press.

AOCS Society. (1998). Official Methods and Recommended Practices of the AOCS, Capillary melting point Cc I-25. Champaign, IL, USA: AOCS Press.

Aronhime, J. S., Sarig, S., \& Garti, N. (1987). Mechanistic considerations of polymorphic transformations of tristearin in the presence of emulsifiers. JAOCS, 64(4), 529-533.

Aronhime, J. S., Sarig, S., \& Garti, N. (1988). Dynamic control of polymorphic transformation in triglycerides by surfactants: the Button Syndrome. JAOCS, 65(7), 1144-1150.

Aronhime, J. S., Sarig, S., \& Garti, N. (1990). Emulsifiers as additives in fats: effect on polymorphic transitions and crystal properties of fatty acids and triglycerides. Food Structure, 9, 337-352.

Arya, G., \& Panagiotopoulos, A. Z. (2005). Log-rolling micelles in sheared amphiphilic thin films. Physical Review Letters, 95(18), 1-4. http://doi.org/10.1103/PhysRevLett.95.188301

Basso, R. C., Ribeiro, A. P. B., Masuchi, M. H., Gioielli, L. A., Gonçalves, L. A. G., Santos, A. O. Dos, Cardoso, L. P., Grimaldi, R. (2010). Tripalmitin and monoacylglycerols as 
modifiers in the crystallisation of palm oil. Food Chemistry, 122(4), 1185-1192. http://doi.org/10.1016/j.foodchem.2010.03.113

Borwankar, R. P., Frye, L. A., Blaurock, A. E., \& Sasevich, F. J. (1992). Rheological characterization of melting of margarines and tablespreads. Journal of Food Engineering, 16(1-2), 55-74. http://doi.org/10.1016/0260-8774(92)90020-7

Callaghan, P. T., \& Jolley, K. W. (1977). An irreversible liquid-liquid phase transition in tristearin. The Journal of Chemical Physics, 67(10), 4773. http://doi.org/10.1063/1.434607

Campos, R., \& Marangoni, A. G. (2014). Crystallization Dynamics of Shear Worked Cocoa Butter. Crystal Growth \& Design, 14(3), 1199-1210. http://doi.org/10.1021/cg4017273

Campos, R., Narine, S. S., \& Marangoni, A. G. (2002). Effect of cooling rate on the structure and mechanical properties of milk fat and lard. Food Research International, 35, 971-981.

Cebula, D. J., McClements, D. J., \& Povey, M. J. W. (1990). Small angle neutron scattering from voids in crystalline trilaurin. Journal of the American Oil Chemists' Society, 67(2), 76-78. http://doi.org/10.1007/BF02540630

Cebula, D. J., Mcclements, D. J., Povey, M. J. W., \& Smith, P. R. (1992). Neutron-Diffraction Studies of Liquid and Crystalline Trilaurin. Journal of the American Oil Chemists Society, 69(2), 130-136. http://doi.org/Doi 10.1007/Bf02540562

Cerdà, J. J., Sintes, T., Holm, C., Sorensen, C. M., \& Chakrabarti, A. (2008). Shear effects on crystal nucleation in colloidal suspensions. Physical Review E - Statistical, Nonlinear, and Soft Matter Physics, 78(3), 1-16. http://doi.org/10.1103/PhysRevE.78.031403

Chaiseri, S., \& Dimick, P. S. (1995). Dynamic crystallization of cocoa butter. II. Morphological, thermal, and chemical characteristics during crystal growth. JAOCS, Journal of the American Oil Chemists'Society, 72(12), 1497-1504. http://doi.org/10.1007/BF02577843

De Graef, V., Dewettinck, K., Verbeken, D., \& Foubert, I. (2006). Rheological behavior of crystallizing palm oil. European Journal of Lipid Science and Technology, 108(10), 864870. http://doi.org/10.1002/ejlt.200600102

De Graef, V., Van Puyvelde, P., Goderis, B., \& Dewettinck, K. (2009). Influence of shear flow on polymorphic behavior and microstructural development during palm oil crystallization. European Journal of Lipid Science and Technology, 111(3), 290-302. http://doi.org/10.1002/ejlt.200800181

Dhonsi, D., \& Stapley, A. G. F. (2006). The effect of shear rate, temperature, sugar and emulsifier on the tempering of cocoa butter. Journal of Food Engineering, 77(4), 936-942. http://doi.org/10.1016/j.jfoodeng.2005.08.022 
Dibildox-Alvarado, E., Laredo, T., Toro-Vazquez, J. F., \& Marangoni, A. G. (2010). Prenucleation structuring of TAG melts revealed by fluorescence polarization spectroscopy and molecular mechanics simulations. JAOCS, Journal of the American Oil Chemists' Society, 87(10), 1115-1125. http://doi.org/10.1007/s11746-010-1596-8

Doi, M., \& Chen, D. (1989). Simulation of aggregating colloids in shear flow. Journal Of Chemical Physics, 90, 5271-5279. http://doi.org/10.1063/1.456430

Douaire, M., Di Bari, V., Norton, J. E., Sullo, A., Lillford, P., \& Norton, I. T. (2014). Fat crystallisation at oil-water interfaces. Advances in Colloid and Interface Science, 203, 1-10. http://doi.org/10.1016/j.cis.2013.10.022

Eisenmann, C., Kim, C., Mattsson, J., \& Weitz, D. A. (2010). Shear melting of a colloidal glass. Physical Review Letters, 104(3), 8-11. http://doi.org/10.1103/PhysRevLett.104.035502

Elisabettini, P., Desmedt, A., \& Durant, F. (1996). Polymorphism of stabilized and nonstabilized tristearin, pure and in the presence of food emulsifiers. Journal of the American Oil Chemists' Society, 73(2), 187-192. http://doi.org/10.1007/BF02523893

Farah, M. A., Oliveira, R. C., Caldas, J. N., \& Rajagopal, K. (2005). Viscosity of water-in-oil emulsions: Variation with temperature and water volume fraction. Journal of Petroleum Science and Engineering, 48(3-4), 169-184. http://doi.org/10.1016/j.petrol.2005.06.014

Garbolino, C., Bartoccini, M., \& Floter, E. (2005). The influence of emulsifiers on the crsytallisation behaviour of a palm oil-based blend. Eur. J. Lipid Sci. Technol., 107.

Garside, J., \& Davey, R. J. (1980). Secondary contact nucleation - Kinetics, growth, and scaleup. In Chemical Engineering Communications (pp. 393-424). Gordon Breach Sci Publ Ltd.

Garti, N., Aronhime, J. S., \& Sarig, S. (1989). The rele of chain length and an emulsifier on the polymorphism of mixtures of triglycerides. Journal of the American Oil Chemists' Society, 66(8), 1085-1089. http://doi.org/10.1007/BF02670089

Ghosh, S., \& Rousseau, D. (2011). Fat crystals and water-in-oil emulsion stability. Current Opinion in Colloid and Interface Science, 16(5), 421-431. http://doi.org/10.1016/j.cocis.2011.06.006

Ghosh, S., \& Rousseau, D. (2012). Triacylglycerol interfacial crystallization and shear structuring in water-in-oil emulsions. Crystal Growth and Design, 12(10), 4944-4954. http://doi.org/10.1021/cg300872m

Ghosh, S., Tran, T., \& Rousseau, D. (2011). Comparison of pickering and network stabilization in water-in-oil emulsions. Langmuir, 27(11), 6589-6597. http://doi.org/10.1021/la200065y 
Gordon, M. H., \& Rahman, I. A. (1991). Effects of minor components on the crystallization of coconut oil. JAOCS, 68(8), 577-579.

Hao, X. T., Chan, N. Y., Heck, C., Tanigaki, N., Paige, M. F., Dunstan, D. E., \& Smith, T. A. (2010). "Log-rolling" alignment in friction-transferred light-emitting conjugated polymer thin films. Macromolecules, 43(24), 10475-10480. http://doi.org/10.1021/ma102137v

Hartel, R. W. (2001). Crystallization in Foods. Gaithersburg, MD, USA: Aspen Publishers, Inc.

Herrera, M. L., \& Hartel, R. W. (2000). Effect of processing conditions on crystallization kinetics of a milk fat model system. JAOCS, 77(11), 1177-1188.

Himawan, C., MacNaughtan, W., Farhat, I.A., \& Stapley, A. G. F. (2007). Polymorphic occurrence and crystallization rates of tristearin/tripalmitin mixtures under non-isothermal conditions. European Journal of Lipid Science and Technology, 109(1), 49-60. http://doi.org/10.1002/ejlt.200600179

Kaufmann, N., De Graef, V., Dewettinck, K., \& Wiking, L. (2012). Shear-induced Crystal Structure Formation in Milk Fat and Blends with Rapeseed Oil. Food Biophysics, 7(4), 308-316. http://doi.org/10.1007/s11483-012-9269-9

Kaufmann, N., Kirkensgaard, J. J. K., Andersen, U., \& Wiking, L. (2013). Shear and rapeseed oil addition affect the crystal polymorphic behavior of milk fat. JAOCS, Journal of the American Oil Chemists'Society, 90(6), 871-880. http://doi.org/10.1007/s11746-013-2226-z

Kellens, M., Meeussen, W., Hammersley, A., \& Reynaers, H. (1991). Synchrotron radiation investigations of the polymorphic transitions in saturated monoacid triglycerides. Part 2: Polymorphism study of a 50:50 mixture of tripalmitin and tristearin during crystallization and melting. Chemistry and Physics of Lipids, 58(1-2), 145-158.

http://doi.org/10.1016/0009-3084(91)90120-Z

Kloek, W., van Vliet, T., \& Walstra, P. (2005). Mechanical properties of fat dispersions prepared in a mechanical crystallizer. Journal of Texture Studies, 36, 544-568.

Lander, B., Seifert, U., \& Speck, T. (2013). Crystallization in a sheared colloidal suspension. Journal of Chemical Physics, 138(22). http://doi.org/10.1063/1.4808354

Lavigne, F., Bourgaux, C., \& Ollivon, M. (1993). Phase transitions of saturated triglycerides. Journal de Physique IV, 3, 137-140. http://doi.org/10.1051/jp4:1993825

Le Révérend, B. J. D., Taylor, M. S., \& Norton, I. T. (2011). Design and application of water-inoil emulsions for use in lipstick formulations. International Journal of Cosmetic Science, 33(3), 263-268. http://doi.org/10.1111/j.1468-2494.2010.00624.x 
Li, Z., Zhou, Z., Armstrong, S. R., Baer, E., Paul, D. R., \& Ellison, C. J. (2014). Multilayer coextrusion of rheologically modified main chain liquid crystalline polymers and resulting orientational order. Polymer, 55(19), 4966-4975.

http://doi.org/10.1016/j.polymer.2014.08.009

Lupi, F. R., Gabriele, D., De Cindio, B., Sánchez, M. C., \& Gallegos, C. (2011). A rheological analysis of structured water-in-olive oil emulsions. Journal of Food Engineering, 107(3-4), 296-303. http://doi.org/10.1016/j.jfoodeng.2011.07.013

Lutton, E. S. (1945). The Polymorphism of Tristearin and Some of its Homologs. Journal of the American Chemical Society, 67(4), 524-527.

MacMillan, S. D., \& Roberts, K. J. (2002). In situ small angle x-ray scattering (SAXS) studies of polymorphism with the associated crystallization of cocoa butter fat using shearing conditions. Crystal Growth \& Design, 2(3), 221-226.

Maleky, F., Acevedo, N. C., \& Marangoni, A. G. (2012). Cooling rate and dilution affect the nanostructure and microstructure differently in model fats. European Journal of Lipid Science and Technology, 114(7), 748-759. http://doi.org/10.1002/ejlt.201100314

Maleky, F., \& Marangoni, A. (2011). Thermal and mechanical properties of cocoa butter crystallized under an external laminar shear field. Crystal Growth \& Design, 11, 24292437.

Maleky, F., \& Marangoni, A. G. (2008). Process development for continuous crystallization of fat under laminar shear. Journal of Food Engineering, 89, 399-407.

Maleky, F., Smith, A. K., \& Marangoni, A. (2011). Laminar shear effects on the crystalline alignments and nanostructure of a triacylglycerol crystal network. Crystal Growth \& Design, 11, 2335-2345.

Malssen, K. Van, Peschar, R., \& Schenk, H. (1996). Real-Time X-Ray Powder Diffraction Investigations on Cocoa Butter . I . Temperature-Dependent. Journal of the American Oil Chemists'Society, 73(10), 1209-1215. http://doi.org/10.1007/BF02525449

Marangoni, A. G., Acevedo, N., Maleky, F., Co, E., Peyronel, F., Mazzanti, G., Quin, B., Pink, D. (2012). Structure and functionality of edible fats. Soft Matter, 8, 1275-1300.

Marangoni, A. G., \& Narine, S. S. (2002). Identifying key structural indicators of mechanical strength in networks of fat crystals. Food Research International, 35(10), 957-969. http://doi.org/10.1016/S0963-9969(02)00158-8

Marangoni, A. G., \& Rousseau, D. (1996). Is plastic fat rheology governed by the fractal nature of the fat crystal network? Journal of the American Oil Chemists' Society, 73(8), 991-994. http://doi.org/10.1007/BF02523406 
Marangoni, A. G., \& Wesdorp, L. H. (2013). Structure and properties of fat crystal networks. Boca Raton, FL, USA: CRC Press Taylor \& Francis Group.

Mazzanti, G., Guthrie, S. E., Sirota, E. B., Marangoni, A. G., \& Idziak, S. H. J. (2003). Orientation and phase transitions of fat crystals under shear. Crystal Growth \& Design, 3(5), 721-725.

Mazzanti, G., Li, M., Marangoni, A. G., \& Idziak, S. H. J. (2011). Effects of shear rate variation on the nanostructure of crystallizing triglycerides. Crystal Growth \& Design, 11, 45444550 .

Mazzanti, G., Marangoni, A. G., \& Idziak, S. H. J. (2005). Modeling phase transitions during the crystallization of a multicomponent fat under shear. Physical Review E, 71, 41607-41612.

Mazzanti, G., Marangoni, A. G., \& Idziak, S. H. J. (2008). Modeling of a two-regime crystallization in a multicomponent lipid system under shear flow. Eur. Phys. J. E, 27, 135144.

McClements, D. J. (2010). Emulsion Design to Improve the Delivery of Functional Lipophilic Components. In M. Doyle \& T. Klaenhammer (Eds.), Annual Review of Food Science and Technology, Vol 1 (pp. 241-269). Annual Reviews, Palo Alto, CA, USA.

Mudge, E. M., \& Mazzanti, G. (2009). Rheo-NMR measurements of cocoa butter crystallized under shear flow. Crystal Growth and Design, 9(7), 3111-3118.

http://doi.org/10.1021/cg800999y

Narine, S. S., \& Humphrey, K. L. (2004). A comparison of lipid shortening functionality as a function of molecular ensemble and shear: Microstructure, polymorphism, solid fat content and texture. Food Research International, 37(1), 28-38.

http://doi.org/10.1016/j.foodres.2003.09.013

Narine, S. S., \& Marangoni, A. G. (1999). Relating structure of fat crystal networks to mechanical properties: A review. Food Research International, 32(4), 227-248. http://doi.org/10.1016/S0963-9969(99)00078-2

Pérez-Martínez, D., Alvarez-Salas, C., Charó-Alonso, M., Dibildox-Alvarado, E., \& ToroVazquez, J. F. (2007). The cooling rate effect on the microstructure and rheological properties of blends of cocoa butter with vegetable oils. Food Research International, 40(1), 47-62. http://doi.org/10.1016/j.foodres.2006.07.016

Peyronel, F., Ilavsky, J., Mazzanti, G., Marangoni, A. G., \& Pink, D. A. (2013). Edible oil structures at low and intermediate concentrations. II. Ultra-small angle X-ray scattering of in situ tristearin solids in triolein. Journal of Applied Physics, 114, 234902-234909.

Peyronel, F., Pink, D. a., \& Marangoni, A. G. (2014). Triglyceride nanocrystal aggregation into polycrystalline colloidal networks: Ultra-small angle X-ray scattering, models and computer 
simulation. Current Opinion in Colloid and Interface Science, 19(5), 459-470.

http://doi.org/10.1016/j.cocis.2014.07.001

Pink, D. A., Quinn, B., Peyronel, F., \& Marangoni, A. G. (2013). Edible oil structures at low and intermediate concentrations. I. Modeling, computer simulation, and predictions for $\mathrm{X}$ ray scattering. Journal of Applied Physics, 114, 234901-234908.

RomoUribe, A., \& Windle, A. H. (1996). Log-rolling"' alignment in main-chain thermotropic liquid crystalline polymer melts under shear: An in-situ WAXS study. Macromolecules, 29(19), 6246-6255. Retrieved from <Go to ISI>://A1996VG24000023

Rousseau, D. (2013). Trends in structuring edible emulsions with Pickering fat crystals. Current Opinion in Colloid and Interface Science, 18(4), 283-291. http://doi.org/10.1016/j.cocis.2013.04.009

Rousseau, D., \& Hodge, S. M. (2005). Stabilization of water-in-oil emulsions with continuous phase crystals. Colloids and Surfaces A: Physicochemical and Engineering Aspects, 260(13), 229-237. http://doi.org/10.1016/j.colsurfa.2005.02.035

Rousseau, D., Hodge, S. M., Nickerson, M. T., \& Paulson, A. T. (2005). Regulating the beta prime to beta polymorphic transition in food fats. JAOCS, 82(1), 7-12.

Sato, K. (2001). Crystallization behavior of fats and lipids - a review. Chemical Engineering Science, 56, 2255-2265.

Shimamura, K., Ueno, S., Miyamoto, Y., \& Sato, K. (2013). Effects of polyglycerine fatty acid esters having different fatty acid moieties on crystallization of palm stearin. Crystal Growth and Design, 13(11), 4746-4754. http://doi.org/10.1021/cg400910g

Smith, K. W., Bhaggan, K., Talbot, G., \& van Malssen, K. F. (2011). Crystallization of fats: influence of minor components and additives. JAOCS, 88, 1085-1101.

Sonwai, S., \& Mackley, M. R. (2006). The effect of shear on the crystallization of cocoa butter. JAOCS, 83(7), 583-596.

Stapley, A. G. F., Tewkesbury, H., \& Fryer, P. J. (1999). The effects of shear and temperature history on the crystallization of chocolate. Journal of the American Oil Chemists' Society, 76(6), 677-685. http://doi.org/10.1007/s11746-999-0159-3

Tang, D., \& Marangoni, A. G. (2006). Microstructure and fractal analysis of fat crystal networks. JAOCS, Journal of the American Oil Chemists' Society, 83(5), 377-388. http://doi.org/10.1007/s11746-006-1216-9 
Tang, D., \& Marangoni, A. G. (2007). Modeling the rheological properties and structure of colloidal fat crystal networks. Trends in Food Science and Technology, 18(9), 474-483. http://doi.org/10.1016/j.tifs.2007.04.015

Tarabukina, E., Jego, F., Haudin, J. M., Navard, P., \& Peuvrel-Disdier, E. (2009). Effect of shear on the rheology and crystallization of palm oil. Journal of Food Science, 74(8), 616-626. http://doi.org/10.1111/j.1750-3841.2009.01304.x

Tran, T., Ghosh, S., \& Rousseau. (2014). Spheroidal fat crystal microstructures formed with confined gap shearing. Cryst. Growth Des., 14, 6383-6390.

Tran, T., Green, N. L., \& Rousseau, D. (2015). Spheroidal fat crystals: structure modification via use of emulsifiers. Crystal Growth \& Design.

Ueno, S., Minato, A., Seto, H., Amemiya, Y., \& Sato, K. (1997). Synchrotron Radiation X-ray Diffraction Study of Liquid Crystal Formation and Polymorphic Crystallization of SOS (sn1,3-Distearoyl-2-oleoyl Glycerol). The Journal of Physical Chemistry B, 101(35), 68476854. http://doi.org/10.1021/jp9715639

Verstringe, S., Danthine, S., Blecker, C., Depypere, F., \& Dewettinck, K. (2013). Influence of monopalmitin on the isothermal crystallization mechanism of palm oil. Food Research International, 51, 344-353.

Verstringe, S., Danthine, S., Blecker, C., \& Dewettinck, K. (2014). Influence of a commercial monoacylglycerol on the crystallization mechanism of palm oil as compared to its pure constituents. Food Research International, 62, 694-700.

Verstringe, S., Dewettinck, K., Ueno, S., \& Sato, K. (2014). Triacylglycerol crystal growth: templating effects of partial glycerols studied with synchrotron radiation microbeam x-ray diffraction. Crystal Growth \& Design, 14, 5219-5226.

Walstra, P. (2003). Physical Chemistry of Foods. New York, USA: CRC Press Taylor \& Francis Group.

Wassell, P., Okamura, A., Young, N. W. G., Bonwick, G., Smith, C., Sato, K., \& Ueno, S. (2012). Synchrotron radiation macrobeam and microbeam $x$-ray diffraction studies of interfacial crystallization of fats in water-in-oil emulsions. Langmuir, 28(13), 5539-5547. http://doi.org/10.1021/la204501t

Wright, A. J., Hartel, R. W., Narine, S. S., \& Marangoni, A. G. (2000). The effect of minor components on milk fat crystallization. Journal of the American Oil Chemists' Society, 77(5), 463-475. http://doi.org/10.1007/s11746-000-0075-8

Zhang, R. C., Xu, Y., Lu, A., Cheng, K., Huang, Y., \& Li, Z. M. (2008). Shear-induced crystallization of poly(phenylene sulfide). Polymer, 49(10), 2604-2613. http://doi.org/10.1016/j.polymer.2008.03.041 
Zhou, Y., \& Hartel, R. W. (2006). Phase behavior of model lipid systems: solubility of highmelting fats in low-melting fats. JAOCS, 83(6), 505-511. 